\title{
An analog of a modular functor from quantized Teichmüller theory
}

\section{J. Teschner}

Institut für theoretische Physik, Freie Universität Berlin,

Arnimallee 14, 14195 Berlin, Germany, and:

Max Planck Institut für Gravitationsphysik (Albert Einstein Institut)

Am Mühlenberg 1, D-14476 Potsdam, Germany

\section{Contents}

I Introduction $\quad 3$

1 Motivation 4

2 Aims 5

3 Overview 6

4 Outlook $\quad 8$

II Coordinates for the Teichmüller spaces 9

5 The Penner coordinates 9

5.1 Triangulations and fat graphs . . . . . . . . . . . . . . . . . 9

5.2 Penner coordinates . . . . . . . . . . . . . . . . . . . 10

5.3 Fock coordinates $\ldots \ldots \ldots \ldots \ldots \ldots \ldots \ldots \ldots \ldots$

6 The Ptolemy groupoids 13

6.1 Groupoids vs. complexes . . . . . . . . . . . . . . . . . . . 13

6.2 Change of the triangulation . . . . . . . . . . . . . . . . . . 13

6.3 The representation of the mapping class group . . . . . . . . . . 15

7 Teichmüller space as the phase space of a constrained system 15

7.1 Kashaev's coordinates . . . . . . . . . . . . . . . . . . . 15

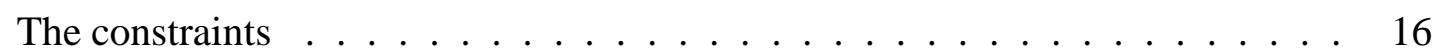

Change of fat graph . . . . . . . . . . . . . . . . . 16 
7.2 The structure of the Kashaev space $W_{\varphi} \ldots \ldots \ldots \ldots \ldots \ldots$

Fock variables vs. Kashaev's variables . . . . . . . . . . . . . . . . . 17

Splitting of $W_{\varphi} \ldots \ldots \ldots \ldots \ldots \ldots \ldots \ldots \ldots$

8 The Fenchel-Nielsen coordinates $\quad 18$

8.1 Definition of the Fenchel-Nielsen coordinates . . . . . . . . . . . . . . . 19

8.2 Symplectic structure . . . . . . . . . . . . . . . . 20

8.3 Geodesic lengths from the Penner coordinates . . . . . . . . . . . . . 20

9 Coordinates for surfaces with holes of finite size 21

9.1 Useful fat graphs on surfaces with holes of finite size . . . . . . . . . . . . . 22

9.2 Kashaev type coordinates . . . . . . . . . . . . . . . . 22

\section{Quantization of the Teichmüller spaces 24}

10 Quantization of the Teichmüller spaces $\quad 24$

10.1 Canonical quantization . . . . . . . . . . . . . . . . . . 24

10.2 Quantization of the Kashaev space $W_{\varphi} \ldots \ldots \ldots \ldots \ldots$

10.3 Reduction to the quantized Teichm üller spaces . . . . . . . . . . . . 26

11 Representations of the mapping class groups 28

11.1 Projective unitary representations of groupoids . . . . . . . . . . . . . . . . . . . . . . 29

11.2 Representations of symmetries of a groupoid . . . . . . . . . . . . . . . . 29

11.3 The projective representation of the Ptolemy groupoid on $\mathcal{K}(\varphi) \ldots \ldots$. . . . 31

11.4 Reduction to the quantized Teichm uller spaces . . . . . . . . . . . . . 31

IV A stable modular functor from the quantum Teichmüller spaces 35

12 The notion of a stable unitary modular functor 35

12.1 Rigged Riemann surfaces . . . . . . . . . . . . . . . . . . . . . . . . . . . . . . . . 36

12.2 Stable unitary modular functors . . . . . . . . . . . . . . . . 36

12.3 Representations of the modular groupoid versus modular functors . . . . . . 38

13 The modular groupoid $\quad 40$

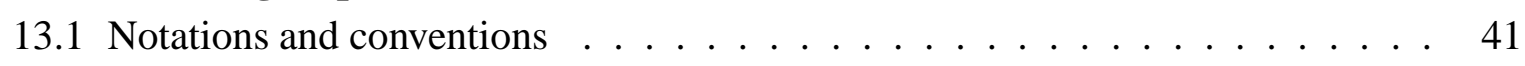

13.2 Generators . . . . . . . . . . . . . . . . . . . 41

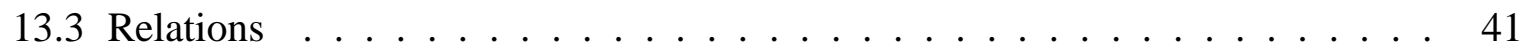

14 From markings to fat graphs $\quad 44$

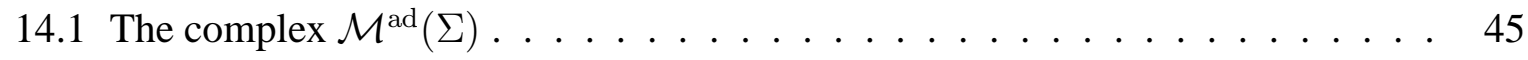

14.2 Separated variables . . . . . . . . . . . . . . . . . . . . . . 47

14.3 Quantized Teichm üller spaces for surfaces with holes . . . . . . . . . . . 48 
15 Geodesic length operators 49

15.1 Overview . . . . . . . . . . . . . . . . . . 49

15.2 Construction of the length operators $\ldots \ldots \ldots \ldots$

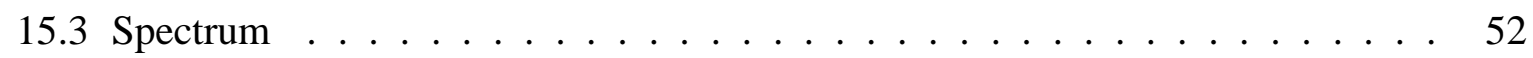

15.4 Relation with the Dehn twist generator . . . . . . . . . . . 53

16 Passage to the length representation $\quad 54$

16.1 The length representation . . . . . . . . . . . . . . . . 54

16.2 Construction of the operator $\mathrm{D}(\sigma) \ldots \ldots \ldots \ldots$

17 Realization of $\mathrm{M}(\Sigma) \quad \mathbf{5 5}$

17.1 Two constructions for the generators . . . . . . . . . . . 55

17.2 The main result . . . . . . . . . . . . . . . . . . . . . 56

17.3 Concluding remarks . . . . . . . . . . . . . 58

A The special functions $e_{b}(x)$ and $s_{b}(x)$

B Operator-theoretical background $\quad 61$

C On the proof of Theorem 2

D Proof of Proposition $10 \quad 63$

E On the proof of Theorem $6 \quad 65$

E.1 A technical preliminary . . . . . . . . . . . . . . 65

E.2 Invariance of length operators $\ldots \ldots \ldots 66$

\section{Part I}

\section{Introduction}

The program of the quantization of the Teichm uller spaces $\mathcal{T}(\Sigma)$ of Riemann surfaces $\Sigma$ which was started in [Fo, CF1] and independently in [Ka1] ${ }^{1}$ is motivated by certain problems and conjectures from mathematical physics. One of the main aims of this program is to construct a one-parameter family of maps

$$
\Sigma \longrightarrow\left(\mathcal{H}_{b}^{\mathcal{T}}(\Sigma), \mathbf{O}_{b}^{\mathcal{T}}(\Sigma), \mathbf{M}_{b}^{\mathcal{T}}(\Sigma)\right), \quad \text { where }
$$

(i) $b$ is a deformation parameter, related to the traditional $\hbar$ via $b=\sqrt{\hbar}$,

(ii) $\Sigma$ is a two-dimensional topological surface possibly with boundary,

(iii) $\mathcal{H}_{b}^{\mathcal{T}}(\Sigma)$ is a Hilbert-space (possibly infinite-dimensional),

(iv) $\mathbf{O}_{b}^{\mathcal{T}}(\Sigma)$ is an algebra of bounded operators on $\mathcal{H}_{b}^{\mathcal{T}}(\Sigma)$ and,

(v) $\mathrm{M}_{b}^{\mathcal{T}}(\Sigma)$ is a unitary projective representation of the mapping class group of $\Sigma$ on $\mathcal{H}_{b}^{\mathcal{T}}(\Sigma)$.

\footnotetext{
${ }^{1}$ See $[\mathrm{FG}, \mathrm{CP}]$ for recent generalizations.
} 
The data $\left(\mathbf{O}_{b}^{\mathcal{T}}(\Sigma), \mathrm{M}_{b}^{\mathcal{T}}(\Sigma)\right)$ are restricted by the requirement that a suitably defined limit of $\mathrm{O}_{b}^{T}(\Sigma)$ for $b \rightarrow 0$ should reproduce the commutative algebra of functions on the Teichm uller space $\mathcal{T}(\Sigma)$, whereas a natural limit $b \rightarrow 0$ of the automorphisms of $\mathbf{O}_{b}^{\mathcal{T}}(\Sigma)$ which are induced by the representation $\mathrm{M}_{b}^{\mathcal{T}}(\Sigma)$ should correspond to the natural action of the mapping class group $\mathrm{MC}(\Sigma)$ on $\mathcal{T}(\Sigma)$.

\section{Motivation}

Motivation for studying this problem comes from mathematical physics. A conjecture of $\mathrm{H}$. Verlinde [V] can be formulated very schematically as the statement that

$$
\left(\mathcal{H}_{b}^{\mathcal{T}}(\Sigma), \mathrm{M}_{b}^{\mathcal{T}}(\Sigma)\right) \simeq\left(\mathcal{H}_{c}^{\mathrm{L}}(\Sigma), \mathrm{M}_{c}^{\mathrm{L}}(\Sigma)\right), \quad \text { where }
$$

(i) $\mathcal{H}_{c}^{\mathrm{L}}(\Sigma)$ has a definition in terms of the representation theory of the Virasoro algebra with central charge $c$ as the so-called space of conformal blocks associated to $\Sigma$, and

(ii) $\mathrm{M}_{c}^{\mathrm{L}}(\Sigma)$ is an action of the mapping class group $\mathrm{MC}(\Sigma)$ on $\mathcal{H}_{c}^{\mathrm{L}}(\Sigma)$ which is canonically associated to the representation-theoretic definition of $\mathcal{H}_{c}^{\mathrm{L}}(\Sigma)$.

Part of the interest in the space $\mathcal{H}_{c}^{\mathrm{L}}(\Sigma)$ from the side of mathematical physics is due to the fact that the elements of $\mathcal{H}_{c}^{\mathrm{L}}(\Sigma)$ represent the basic building blocks in the so-called Liouville conformal field theory [TL1]. Deep connections between the perturbative approach to quantum Liouville theory on the one hand and Teichm uller theory on the other hand have been exhibited by Takhtajan, Zograf and Teo see e.g. [TT] and references therein.

This conjecture may be seen as a non-compact analog of similar relations between the quantization of moduli spaces of flat connections on Riemann surfaces on the one hand, and rational conformal field theories on the other hand. For K being a compact group, the geometric quantization of the moduli space $\mathcal{M}_{\mathrm{K}}(\Sigma)$ of flat K-connections on a Riemann surface was performed in [Hi2, ADPW]. Alternative approaches were based on more explicit descriptions of the symplectic structure on $\mathcal{M}_{\mathrm{K}}(\Sigma)$ [FR, AGS, BR, AMR]. ${ }^{2}$ In either case one may schematically describe one of the main results of these constructions as an assignment

$$
\Sigma \longrightarrow\left(\mathcal{H}_{k}^{\mathcal{M}}(\Sigma), \mathrm{M}_{k}^{\mathcal{M}}(\Sigma)\right), \quad \text { where }
$$

(i) $\mathcal{H}_{k}^{\mathcal{M}}(\Sigma)$ is a finite-dimensional vector space,

(ii) $\mathrm{M}_{k}^{\mathcal{M}}(\Sigma)$ is a projective representation of the mapping class group of $\Sigma$ on $\mathcal{H}_{k}^{\mathcal{M}}(\Sigma)$.

Part of the interest in these results was due to the close relations between the representation $\mathrm{M}_{k}^{\mathcal{M}}(\Sigma)$ and the Reshetikhin-Turaev invariants of three manifolds [RT]. Another source of interest were the relations to rational conformal field theory, which were predicted in [Wi], see

\footnotetext{
${ }^{2}$ The equivalence between the different quantization schemes has not been discussed in detail so far. It boils down to the verifi cation that the monodromy representation of the KZ-connection constructed within the geometric quantization framework of [Hi2, ADPW] is equivalent to the mapping class group representation defi ned in [AGS, $\mathrm{BR}]$. It seems that e.g. combining the results of [La] and [BK2] does the job.
} 
[So] for a review of mathematical approaches to the problem and further references. These relations may, again schematically, be summarized as the existence of canonical isomorphisms

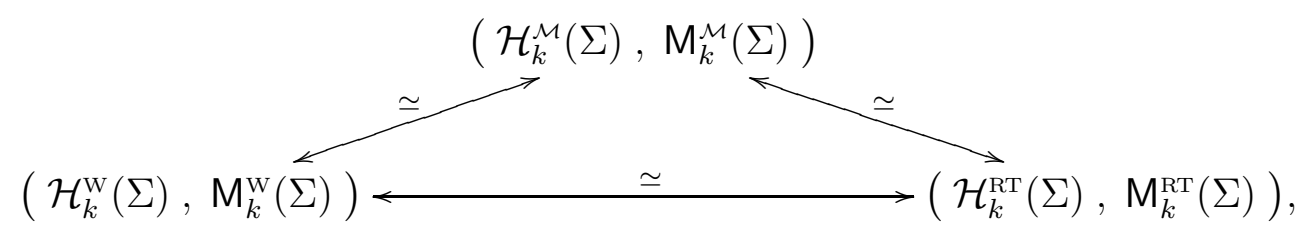

where

(i) $\mathcal{H}_{k}^{\mathrm{w}}(\Sigma)$ is the space of conformal blocks in the WZNW-model associated to the compact group $\mathrm{K}$, which can be defined in terms of the representation theory of the affine Lie algebra $\hat{\mathfrak{g}}_{k}$ with level $k$ associated to the Lie algebra $\mathfrak{g}$ of $K$,

(ii) $\mathrm{M}_{k}^{\mathrm{w}}(\Sigma)$ is the natural action of the mapping class group on $\mathcal{H}_{k}^{\mathrm{w}}(\Sigma)$, which can be defined by means of the monodromy representation of the Knizhnik-Zamolodchikov connection,

(iii) $\mathcal{H}_{k}^{\mathrm{RT}}(\Sigma)$ is the space of invariants in certain tensor products of representations of the quantum group $\mathcal{U}_{q}(\mathfrak{g})$

(iv) $\mathrm{M}_{k}^{\mathrm{RT}}(\Sigma)$ is the mapping class group representation on $\mathcal{H}_{k}^{\mathrm{RT}}(\Sigma)$ defined by the construction of Reshetikhin-Turaev.

The quantization program (0.1) can be seen as a non-compact analog of (1.3) in the following sense. In (0.1) the role of the moduli space of flat connections $\mathcal{M}_{\mathrm{K}}(\Sigma)$ is taken by the Teichm uller space $\mathcal{T}(\Sigma)$, which can be identified with the component in the moduli space $\mathcal{M}_{\mathrm{G}}(\Sigma)$ of flat $G=S L(2, \mathbb{R})$-connections that has maximal Euler class [Hi1, Go2]. Moreover, the natural symplectic structure on the moduli space of flat $S L(2, \mathbb{R})$-connections restricts to the WeilPetersson symplectic form on $\mathcal{T}(\Sigma)$ [Go1]. A quantization of the Teichm uller space $\mathcal{T}(\Sigma)$ may therefore be regarded as providing a quantization of a topological component in the moduli space $\mathcal{M}_{\mathrm{G}}(\Sigma)$.

We expect that any non-compact counterpart of the developments mentioned above will be mathematically at least as rich as the already known results associated to compact groups $\mathrm{K}$. In particular we expect that certain analogs of the constructions of Reshetikhin-Turaev and/or Turaev-Viro will capture information on the geometry of hyperbolic three manifolds, similar and probably related to the appearance of hyperbolic volumes in the asymptotic behavior of certain link invariants $[\mathrm{KaV}]$.

\section{Aims}

A major step towards establishing H. Verlinde's conjecture (1.2) is to show that the quantization of the Teichm uller spaces (0.1) as initiated in [Fo, CF1], [Ka1] produces an analog of a modular functor. The basic data of a modular functor are assignments such as (1.3), which are required to satisfy a natural set of axioms as discussed in Section 12. One of the most important implications of the axioms of a modular functor are simple relations between the representations of the mapping class groups associated to $\Sigma$ and $\Sigma^{\dagger c}$ respectively, where $\Sigma^{\dagger c}$ is the surface that is obtained from $\Sigma$ by cutting along a simple closed curve $c$. These relations imply that the 
representation $\mathrm{M}_{k}^{\mathcal{M}}(\Sigma)$ restricts to - and is generated by - the representations $\mathrm{M}_{k}^{\mathcal{M}}\left(\Sigma^{\prime}\right)$ which are associated to those subsurfaces $\Sigma^{\prime}$ that can be obtained from $\Sigma$ by cutting along a set of nonintersecting simple closed curves. This crucial locality property can be seen as the hard core of the notion of a modular functor.

Within the formalisms introduced in [Fo, CF1], [Ka1]-[Ka3] it is far from obvious that the quantization of Teichm uller spaces constructed there has such properties. To show that this is indeed the case is the main problem solved in this paper. The representation $\mathrm{M}_{b}^{\mathcal{T}}(\Sigma)$ constructed and investigated in [Ka1]-[Ka3] is obtained by exploiting the fact that the mapping class group can be embedded into the so-called Ptolemy groupoid associated to the transformations between different triangulations of a Riemann surface $\Sigma$. A representation of the Ptolemy groupoid is constructed in [Ka1]-[Ka3], which then canonically induces a projective unitary representation of the mapping class group $\operatorname{MC}(\Sigma)$. The simplicity of the Ptolemy groupoid, which underlies the elegance of the constructions in [Ka1]-[Ka3] now turns out to cause a major problem from the point of view of our aims, since the above-mentioned locality properties implied in the notion of a modular functor are not transparently realized by the Ptolemy groupoid.

Essentially our task is therefore to go from triangulations to pants decompositions, which is the type of decomposition of a Riemann surface $\Sigma$ that is naturally associated to the concept of a modular functor. This requires to construct a change of representation for $\mathcal{H}_{b}^{\mathcal{T}}(\Sigma)$ from the one naturally associated to triangulations of $\Sigma$ [Ka1]-[Ka3] to another one which is associated to pants decompositions. The main tool for doing this are the geodesic length operators introduced and studied in [Fo, CF2] and [Ka3, Ka4], which are the observables on the quantized Teich$m$ uller spaces that are associated with the geodesic length functions ${ }^{3}$ on the classical Teich$m$ üller spaces. The length operators associated to a maximal set of non-intersecting simple closed curves turn out to furnish a set of commuting self-adjoint operators, and the simultaneous diagonalization of these operators defines the sought-for change of representation.

There is a natural groupoid associated with the transformations between different pants decompositions. Of particular importance for us will be a certain refinement of this groupoid which will be called the modular groupoid $\mathrm{M}(\Sigma)$. The modular groupoid $\mathrm{M}(\Sigma)$ has been introduced for the study of rational conformal field theories by Moore and Seiberg in [MS], and it was further studied in particular in [BK1]. Constructing a modular functor is essentially equivalent to constructing a tower of representations of the modular groupoid. Our main aim in the present paper will be to show that the quantization of Teichm uller spaces allows one to construct a tower of representations of the modular groupoid by unitary operators in a natural way.

\section{Overview}

This paper has three main parts. The first of these parts collects the necessary results from the "classical" theory of Riemann surfaces. This includes a review of two types of coordinate systems for the Teichm üller spaces $\mathcal{T}(\Sigma)$, one of which is associated to triangulations of $\Sigma$, the other to pants decompositions. The coordinates associated to triangulations were first in-

\footnotetext{
${ }^{3}$ See [Wo1, Wo2, Wo3] for some classical work on the symplectic nature of the Fenchel-Nielsen coordinates which represents important background for our results.
} 
troduced by Penner in [P1]. We will also need to discuss variants of these coordinates due to Fock [Fo] and Kashaev [Ka1] respectively. The changes of the underlying triangulation of $\Sigma$ generate a groupoid, the Ptolemy groupoid $\operatorname{Pt}(\Sigma)$, which has a useful representation in terms of generators and relations (Theorem 2).

The coordinates associated to pants decompositions are the classical Fenchel-Nielsen coordinates, which we review briefly in $\S 8.1$. We furthermore explain how the coordinates of Penner [P1] and Kashaev [Ka1], which were originally introduced to parameterize the Teichm uller spaces of surfaces $\Sigma$ with punctures only, can be used to provide coordinates also for the case where the surface $\Sigma$ has holes represented by geodesics of finite length.

The material in this part is mostly known, but it is scattered over many places in the literature, and some basic results were stated in the original references without a proof. We have therefore tried to give a reasonably self-contained and complete presentation of the relevant material, providing proofs where these are not available elsewhere.

The second part gives a largely self-contained presentation of the foundations of the quantization of Teichm uller spaces. Our presentation is heavily inspired by [Ka1]-[Ka3], but we deviate from these references in some important points. The treatment presented in this paper seems to be the first complete and mathematically rigorous formulation of the quantum theory of the Teichm üller spaces.

The main aims of this paper are finally achieved in the third part. We begin in $\S 12$ by introducing the notion of a stable unitary modular functor, and by explaining why having a stable unitary modular functor is equivalent to having a tower of unitary projective representations of the modular groupoid.

In $\S 13$ we will reformulate the main result of [MS, BK1] concerning the description of $\mathrm{M}(\Sigma)$ in terms of generators and relations in a way that is convenient for us.

Of particular importance for us will be $\S 14$, where important first relations between certain subgroupoids of $\mathrm{M}(\Sigma)$ and $\operatorname{Pt}(\Sigma)$ are observed.

In $\S 15$ we define the geodesic length operators and establish their main properties. These results are of independent interest since some important properties of the geodesic length operators had not been proven in full generality before.

A key step in our constructions is taken in $\S 16$ by constructing a change of representation from the original one to a representation in which the length operators associated to a pants decomposition are simultaneously diagonalized. An important feature of this construction is the fact that the unitary operator which describes the change of representation factorizes into operators associated to the individual three holed spheres (trinions) which appear in a pants decomposition.

In $\S 17$ we construct the corresponding representation of the modular groupoid $\mathrm{M}(\Sigma)$. The operators which represent $\mathrm{M}(\Sigma)$ are constructed out of compositions of the representatives for the transformations in $\operatorname{Pt}(\Sigma)$. This makes it relatively easy to verify the relations of $\mathrm{M}(\Sigma)$, but the price to pay is that some crucial locality properties are more difficult to prove. 


\section{Outlook}

In a sequel [TT2] to this paper we will calculate the matrix coefficients of the operators which generate the representation of the modular groupoid explicitly. A close relation to the modular double $\mathcal{D U}_{q}(\operatorname{sl}(2, \mathbb{R}))$ of $\mathcal{U}_{q}(\operatorname{sl}(2, \mathbb{R}))$ as defined and studied in [Fa, PT, BT] will be found.

It should be noted that $\left(\mathcal{H}_{b}^{\mathcal{T}}(\Sigma), \mathrm{M}_{b}^{\mathcal{T}}(\Sigma)\right)$ will not satisfy all the usual axioms of a modular functor, which require, in particular, that the vector space $\mathcal{V}(\Sigma)$ assigned to each Riemann surface should be finite-dimensional. Most importantly, however, the assignment $\Sigma \rightarrow$ $\left(\mathcal{H}_{b}^{\mathcal{T}}(\Sigma), \mathrm{M}_{b}^{\mathcal{T}}(\Sigma)\right)$ was up to now only constructed for surfaces $\Sigma$ which have at least one boundary component.

What will allow us to overcome this unsatisfactory feature are the remarkable analytic properties that the matrix coefficients of the operators which represent the modular groupoid will be shown to have. It turns out that the mapping class group representation $\mathrm{M}_{b}^{\mathcal{T}}(\Sigma)$ assigned to a Riemann surface with a boundary represented by geodesics of a fixed length depends analytically on the values of these lengths. The analytic properties of the matrix coefficients will furthermore allow us to "close a hole" by taking a limit where the length parameter assigned to this boundary component approaches a certain imaginary value. It will be shown in [TT2] that the resulting mapping class group representation is equivalent to the one on the surface which is obtained by gluing a disc into the relevant boundary component.

Concerning the representation theoretic side of H. Verlinde's conjecture (1.2) it should be mentioned that a complete mathematical construction of $\left(\mathcal{H}_{c}^{\mathrm{L}}(\Sigma), \mathrm{M}_{c}^{\mathrm{L}}(\Sigma)\right)$ is not available so far, but nontrivial steps in the direction of constructing and describing $\left(\mathcal{H}_{c}^{\mathrm{L}}(\Sigma), \mathrm{M}_{c}^{\mathrm{L}}(\Sigma)\right)$ precisely have been taken in [TL2] in the case of surfaces $\Sigma$ of genus zero. This includes in particular the derivation of explicit formulae for a set of basic data which characterize the resulting representation of the braid group uniquely.

The explicit computation of the matrix coefficients of the operators which generate the representation of the modular groupoid carried out in [TT2] will therefore allow us to verify $\mathrm{H}$. Verlinde's conjecture (1.2) in the case of Riemann surfaces of genus zero.

We furthermore expect that it should be possible to construct non-compact analogs of $\left(\mathcal{H}_{k}^{\mathrm{RT}}(\Sigma), \mathrm{M}_{k}^{\mathrm{RT}}(\Sigma)\right)$ based the non-compact quantum group $\mathcal{D U}_{q}(s l(2, \mathbb{R}))$, and thereby complete a non-compact analog of the triangle (1.4).

\section{Acknowledgements}

The author would like to thank L. Chekhov, V. Fock and especially R. Kashaev for useful discussions.

We furthermore gratefully acknowledge financial support from the DFG by a Heisenberg fellowship, as well as the kind hospitality of the Humboldt University Berlin, the School of Natural Sciences of the IAS Princeton, the Physics Department of Chicago University and the Caltech, Pasadena. 


\section{Part II}

\section{Coordinates for the Teichmüller spaces}

We will consider two-dimensional surfaces $\Sigma$ with genus $g \geq 0$ and $s \geq 1$ boundary components such that

$$
M \equiv 2 g-2+s>0 .
$$

On $\Sigma$ we will consider metrics of constant negative curvature -1 . Our main interest will be the case where the boundary components can be represented by geodesics of finite length. Such boundary components will also be called holes in the following. However, to begin with we will focus on the case where the boundary components are punctures, i.e. holes of vanishing geodesic circumference.

The space of deformations of the metrics of constant negative curvature is called the Teichm uller space $\mathcal{T}(\Sigma)$. It will be of basic importance for us to have useful systems of coordinates for $\mathcal{T}(\Sigma)$.

We will consider two classes of coordinate systems which are associated to two types of graphs drawn on the Riemann surfaces respectively. The first class of coordinates goes back to Penner and is associated to triangulations of the Riemann surface or the corresponding dual graphs, the so-called fat graphs. We will also describe two useful variants of the Penner coordinates due to Kashaev and Fock respectively.

The second class of coordinates are the classical Fenchel-Nielsen length-twist coordinates. One may view them as being associated to a second type of graph on a surface $\Sigma$ called marking that determines in particular a decomposition of the surface into three-holed spheres (trinions).

In the following first part of this paper we shall describe these coordinate systems in some detail, discuss the graphs on $\Sigma$ that these coordinates are associated to, as well as the groupoids generated by the transformations between different choices of these graphs.

\section{The Penner coordinates}

\subsection{Triangulations and fat graphs}

Consider a fixed oriented topological surface $\Sigma$ of genus $g$ with $s \geq 1$ punctures. An ideal triangulation $\tau$ of $\Sigma$ is the isotopy class of a collection of disjointly embedded arcs in $\Sigma$ running between the punctures such that $\tau$ decomposes $\Sigma$ into triangles. There are $2 M$ triangles and $3 M$ edges for any ideal triangulation. As an example we have drawn a triangulation of the once-punctured torus in Figure 1.

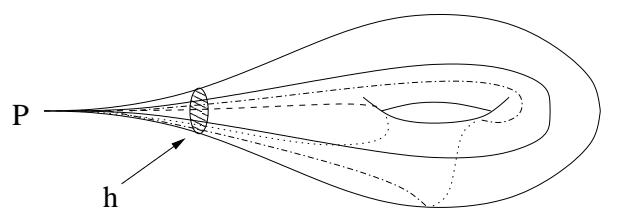

Figure 1: Triangulation of the once-punctured torus. 
The graph dual to a triangulation is a trivalent fat graph i.e. a trivalent graph embedded in the surface with fixed cyclic order of the edges incident to each vertex. An example for a fat graph on the once-punctured torus is depicted in Figure 2. The sets of vertices and edges of a

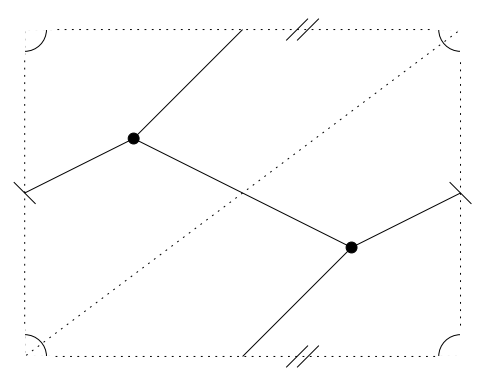

Figure 2: Another representation of the triangulation from Figure 1 and the dual fat graph.

fat graph $\varphi$ will be denoted $\varphi_{\mathrm{o}}$ and $\varphi_{1}$ respectively.

An ideal triangulation is called decorated if the triangles are numbered and if a corner is marked for each triangle [Ka1]. The decoration of the triangle $t_{v}$ dual to a vertex $v \in \varphi_{\mathrm{o}}$ can be used to fix a numbering convention for the edges $e_{i}^{v}, i=1,2,3$ which emanate from $v$ as defined in Figure 3.

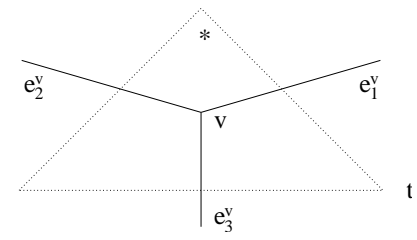

Figure 3: Graphical representation of the vertex $v$ dual to a triangle $t$. The marked corner defines a corresponding numbering of the edges that emanate at $v$.

Remark 1. Decorated ideal triangulations are dual to decorated fat graphs, which means that the vertices are numbered, and for each vertex $v \in \varphi_{\mathrm{o}}$ one has chosen a distinguished edge $e_{v} \in \varphi_{1}$. As a convention we will assume that fat graphs always carry such a decoration unless otherwise stated.

\subsection{Penner coordinates}

It turns out to be useful to consider a somewhat enlarged object which keeps track of the choices of horocycles around each of the punctures, the so-called decorated Teichm üller space $\tilde{\mathcal{T}}(\Sigma)$. $\tilde{\mathcal{T}}(\Sigma)$ is defined as a principal $\mathbb{R}_{+}^{s}$-bundle over $\mathcal{T}(\Sigma)$ by taking the s-tuple of horocycles around each of the punctures as the fiber over a point of $\mathcal{T}(\Sigma)$. The ordered s-tuple of hyperbolic lengths of the horocycles gives coordinates for the fibers.

Given any point $P$ in the decorated Teichm üller space $\widetilde{\mathcal{T}}(\Sigma)$ and an ideal triangulation $\tau$ of $\Sigma$, Penner assigns a coordinate value to each of the edges in $\tau$ by means of the following construction. By means of Fuchsian uniformization one may equip the surface $\Sigma$ with a unique 


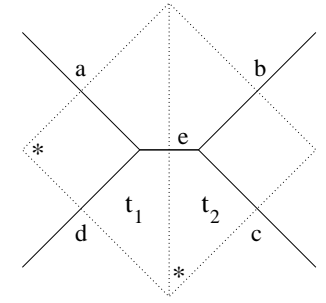

Figure 4: Two adjacent triangles and the dual fat graph.

hyperbolic metric $g$ associated to our chosen point $P \in \widetilde{\mathcal{T}}(\Sigma)$. Let $\tau_{1}$ be the set of edges of a triangulation $\tau$. Each edge $e$ in $\tau_{\perp}$ may be straightened to a geodesic for the hyperbolic metric $g$. The coordinate $l_{e}(P)$ is defined as the hyperbolic length of the segment of $e$ that lies between the two horocycles surrounding the punctures that $e$ connects, taken with positive sign if the two horocycles are disjoint, with negative sign otherwise. We are going to consider the tuple $\left(l_{e}\right)_{e \in \tau_{\perp}}$ as a vector in the vector space $\mathbb{R}^{\tau_{\perp}}$ of dimension $3 M$.

Theorem 1. Penner [P1][P2]

(a) For any fixed ideal triangulation $\tau$ of $\Sigma$, the function

$$
l: \tilde{\mathcal{T}}(\Sigma) \rightarrow \mathbb{R}^{\tau_{1}}, \quad P \rightarrow\left(l_{e}(P)\right)_{e \in \tau_{1}}
$$

is a homeomorphism.

(b) The pull-back of the Weil-Petersson two-form $\omega$ on $\mathcal{T}(\Sigma)$ is given by the expression

$$
\omega=-\sum_{t \in \tau_{2}}\left(d l_{e_{1}(t)} \wedge d l_{e_{2}(t)}+d l_{e_{2}(t)} \wedge d l_{e_{3}(t)}+d l_{e_{3}(t)} \wedge d l_{e_{1}(t)}\right),
$$

where the summation is extended over the set $\tau_{2}$ of triangles of $\tau$, and $e_{i}(t), i=1,2,3$ are the edges bounding the triangle $t$, labelled in the counter-clockwise sense.

The Teichm uller space $\mathcal{T}(\Sigma)$ itself can finally be described as the space of orbits in $\widetilde{\mathcal{T}}(\Sigma)$ under the following symmetry. Choose a number $d(\mathfrak{p})$ for each puncture $\mathfrak{p}$. Let the action of the symmetry be defined by

$$
l_{e}^{\prime} \equiv l_{e}+d(\mathfrak{p})+d\left(\mathfrak{p}^{\prime}\right)
$$

if the edge $e$ connects the punctures $\mathfrak{p}$ and $\mathfrak{p}^{\prime}$.

\subsection{Fock coordinates}

There is a useful variant of the Penner coordinates which was introduced by V. Fock in [Fo]. In terms of the Penner coordinates one may define the Fock coordinates in terms of certain crossratios. Given a quadrilateral formed by two adjacent triangles we are going to keep the labelling of the edges introduced in Figure 4. Let

$$
z_{e}=l_{a}+l_{c}-l_{b}-l_{d}
$$

The dependence of the Penner coordinates on the choice of horocycles drops out in the Fock coordinates. However, the variables $z_{e}$ assigned to the $3 M$ edges in a triangulation are not all 
independent. To describe the relations that they satisfy it is convenient to think of the Fock coordinates as being assigned to the edges of the fat graph dual to the given triangulation. Each closed curve $c$ on $\Sigma$ is homotopic to a unique path $g_{c}$ on the fat graph which has minimal length w.r.t. the metric defined by assigning each edge of $\varphi$ the length one. Such paths will also be called graph geodesics in the following. The path $g_{c}$ may be described by a sequence of edges $e_{1}^{c}, \ldots, e_{n_{c}}^{c} \in \varphi_{1}$. To a closed curve $c$ let us associate

$$
f_{\varphi, c} \equiv \sum_{i=1}^{n_{c}} z_{e_{i}^{c}}
$$

The definition (5.2) then implies the relations

$$
f_{\varphi, c}=0
$$

for any closed curve $c$ that encircles one puncture only. If one uses the equations (5.4) to express $s$ of the variables $z_{e}$ in terms of the others one obtains a set of coordinates for $\mathcal{T}(\Sigma)$.

On the $3 M$-dimensional space $F_{\varphi}$ that is spanned by the coordinate functions $z_{e}(P)$ one may define a Poisson bracket $\Omega_{\mathrm{WP}}$ which is such that

(i) the elements $f_{a}, a=1, \ldots, s$ span the set $C_{\varphi}$ of all $\mathfrak{c} \in F_{\varphi}$ such that

$$
\Omega_{\mathrm{WP}}(\mathfrak{c}, \mathfrak{w})=0 \quad \text { for all } \mathfrak{w} \in F_{\varphi}
$$

(ii) the Poisson bracket which is induced by $\Omega_{\mathrm{WP}}$ on the quotient $F_{\varphi} / C_{\varphi}$ coincides with the Poisson bracket which corresponds to the Weil-Petersson symplectic form on $\mathcal{T}(\Sigma)$.

There exists a rather simple description for this Poisson-bracket [Fo]:

$$
\Omega_{\mathrm{WP}}\left(z_{e}, z_{e^{\prime}}\right)=n_{e, e^{\prime}}, \quad \text { where } \quad n_{e, e^{\prime}} \in\{-2,-1,0,1,2\} .
$$

The value of $n_{e, e^{\prime}}$ depends on how edges $e$ and $e^{\prime}$ are embedded into a given fat graph. If $e$ and $e^{\prime}$ don't have a common vertex at their ends, or if one of $e, e^{\prime}$ starts and ends at the same vertex then $n_{e, e^{\prime}}=0$. In the case that $e$ and $e^{\prime}$ meet at two vertices one has $n_{e, e^{\prime}}=2\left(\right.$ resp. $\left.n_{e, e^{\prime}}=-2\right)$ if $e^{\prime}$ is the first edge to the right ${ }^{4}$ (resp. left) of $e$ at both vertices, and $n_{e, e^{\prime}}=0$ otherwise. In all the remaining cases $n_{e, e^{\prime}}=1$ (resp. $n_{e, e^{\prime}}=-1$ ) if $e^{\prime}$ is the first edge to the right (resp. left) of $e$ at the common vertex.

The coordinates $z_{e}$ also have a nice geometrical meaning [Fo]. In the Fuchsian uniformization the two triangles that share the common edge $e$ will be mapped into ideal hyperbolic triangles in the upper half plane. The edges are then represented by half-circles, and the corners will be at points $x_{1}, \ldots, x_{4}$ on the real line, see Figure 5.

We then have

$$
\exp \left(z_{e}\right)=\frac{\left(x_{4}-x_{1}\right)\left(x_{3}-x_{2}\right)}{\left(x_{4}-x_{3}\right)\left(x_{2}-x_{1}\right)} .
$$

By means of M"obius-transformations $x_{i} \rightarrow \frac{a x_{i}+b}{c x_{i}+d}$ one may map the corners of one of the two triangles to $-1,0$ and $\infty$ respectively. The variable $z_{e}$, being expressed in terms of the M"obiusinvariant cross-ratio therefore parameterizes the different ways of gluing two ideal hyperbolic

\footnotetext{
${ }^{4}$ w.r.t. to the orientation induced by the embedding of the fat-graph into the surface
} 


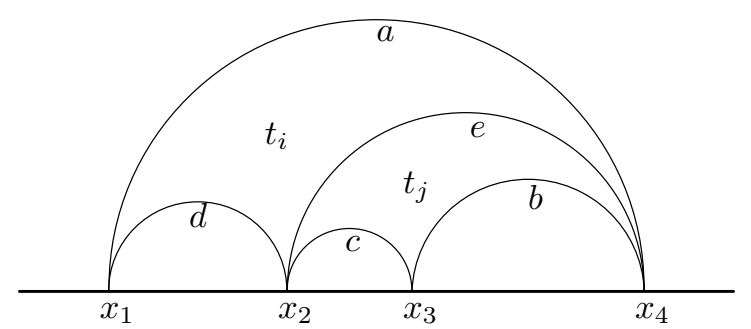

Figure 5: Representation of the triangles $t_{i}$ and $t_{j}$ in the upper half plane.

triangles along a common edge modulo M"obius-transformations. Given the variables $z_{e}$ one may reconstruct the Riemann surface as represented in the Fuchsian uniformization by successively mapping ideal hyperbolic triangles into the upper half-plane, glued along the edges $e$ in the way prescribed by the given value $z_{e}[\mathrm{Fo}]$.

\section{The Ptolemy groupoids}

In the previous section we had associated coordinate systems to fat graphs on a surface $\Sigma$ : A change of graph will of course induce a change of coordinates. The groupoid generated by the moves between different fat graphs will be the subject of the present section.

\subsection{Groupoids vs. complexes}

The groupoids that we will be interested in can be conveniently described as 2-dimensional connected $\mathrm{CW}$ complexes $\mathcal{G}$. The set of vertices $\mathcal{G}_{\mathrm{o}}$ of $\mathcal{G}$ will be represented by certain sets of graphs, within this section called fat graphs. The (directed) edges $E \in \mathcal{G}_{1}$ that connect these vertices correspond to the generators ("elementary moves") of the groupoid, while the faces $F \in \mathcal{G}_{2}$ of $\mathcal{G}$ yield the relations.

The groupoid $\mathrm{G}$ associated to the 2-dimensional connected $\mathrm{CW}$ complexes $\mathcal{G}$ will then simply be the path groupoid of $\mathcal{G}$, which has the vertices in $\mathcal{G}_{0}$ as objects and the homotopy classes of edge paths between two vertices as morphisms. The homotopy class of paths leading from vertex $V_{1} \in \mathcal{G}_{\mathrm{o}}$ to vertex $V_{2} \in \mathcal{G}_{\mathrm{o}}$ will be denoted by $\left[V_{2}, V_{1}\right]$. Similarly we will sometimes use the notation $\left[W_{E}, V_{E}\right]$ for the element of $\mathrm{G}$ which corresponds to an edge $E \in \mathcal{G}_{1}$.

A path $\pi$ which represents an element in the homotopy class $[W, V]$ may be represented by a chain of edges $E \in \mathcal{G}_{1}$, i.e. an ordered sequence $\left(E_{\pi, n(\pi)}, \ldots, E_{\pi, 1}\right), E_{\pi, \jmath} \in \mathcal{G}_{1}$ for $\jmath=$ $1, \ldots, n(\pi)$ such that $E_{\pi, \jmath} \in\left[V_{\pi, \jmath+1}, V_{\pi, \jmath}\right]$ for $\jmath=1, \ldots, n(\pi)-1$, and $V_{\pi, 1}=V, V_{\pi, n(\pi)}=W$. We will also use the suggestive notation $E_{n} \circ E_{n-1} \circ \cdots \circ E_{1}$ to denote a chain.

\subsection{Change of the triangulation}

In the case of the Ptolemy groupoids $\operatorname{Pt}(\Sigma)$ we will consider a complex $\mathcal{P} t(\Sigma)$, where the set $\mathcal{G}_{\mathrm{o}}=\mathcal{P} t_{\mathrm{o}}(\Sigma)$ is defined to be the set of fat graphs on $\Sigma$. Let us furthermore define $\mathcal{P} t_{\mathbf{1}}(\Sigma)$ to consist of the following elementary moves.

(i) Permutation ( $v w)$ : Exchanges the labels of the vertices $v$ and $w$.

(ii) Rotation $\rho_{v}$ : See Figure 6. 


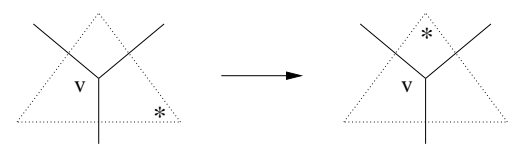

Figure 6: Transformation $\rho_{v}$ changes the marked corner of the triangle dual to a vertex $v \in \varphi_{\mathrm{o}}$. (iii) Flip $\omega_{v w}$ : See Figure 7.

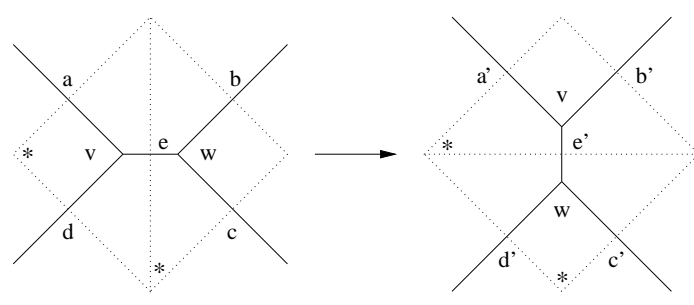

Figure 7: The flip transformation $\omega_{v w}$ changes the diagonal in the quadrilateral formed by the two adjacent triangles $t_{v}$ and $t_{w}$.

Proposition 1. - [P1, Proposition 7.1][Ka3] -

The complex $\mathcal{P} t(\Sigma)$ is connected, i.e. for a given surface $\Sigma$, any two fat graphs $\varphi$ and $\varphi^{\prime}$ can be connected by a chain of elementary transformations.

Validity of the following relations in $\operatorname{Pt}(\Sigma)$ can easily be verified pictorially [Ka3].

$$
\begin{aligned}
& \rho_{v} \circ \rho_{v} \circ \rho_{v}=i d, \\
& \omega_{u_{1} u_{2}} \circ \omega_{u_{3} u_{4}}=\omega_{u_{3} u_{4}} \circ \omega_{u_{1} u_{2}}, \quad u_{r} \neq u_{s} \text { for } r \neq s, \\
& \omega_{v w} \circ \omega_{u w} \circ \omega_{u v}=\omega_{u v} \circ \omega_{v w}, \\
& \left(\rho_{v}^{-1} \times \rho_{w}\right) \circ \omega_{v w}=\omega_{w v} \circ\left(\rho_{v}^{-1} \times \rho_{w}\right), \\
& \omega_{w v} \circ \rho_{v} \circ \omega_{v w}=(v w) \circ\left(\rho_{v} \times \rho_{w}\right) .
\end{aligned}
$$

Theorem 2. The complex $\mathcal{P} t(\Sigma)$ is simply connected, i.e. any relation between the generators $(v w), \rho_{v}$ and $\omega_{v w}$ of the Ptolemy groupoid is a consequence of the relations (6.1) together with the relations of the permutation group.

The proof of this theorem is explained in Appendix C.

One of the main virtues of the Penner coordinates is that the corresponding change of coordinates can be described rather simply.

Lemma 1. - Lemma A.1a of [P2] -

Let $\tau^{\prime}$ be the triangulation obtained by applying the flip of Figure 7 to a pair of adjacent triangles in a given initial triangulation $\tau$, and denote $e$ and $e^{\prime}$ the diagonal edge before and after the flip. The coordinates associated to $\tau$ and $\tau^{\prime}$ will then agree for each edge that the two triangulations have in common, and

$$
\lambda_{e^{\prime}}=\frac{1}{\lambda_{e}}\left(\lambda_{a} \lambda_{c}+\lambda_{b} \lambda_{d}\right), \quad \lambda_{f} \equiv \sqrt{2} \exp \left(\frac{1}{2} l_{f}\right), \quad \forall f \in \varphi_{\mathbf{1}},
$$

where we have labelled the edges according to Figure 7. 
The corresponding transformation of the Fock variables is also easy to describe:

$$
\begin{aligned}
& e^{-z_{a^{\prime}}}=e^{-z_{a}}\left(1+e^{-z_{e}}\right) \quad z_{e^{\prime}}=-z_{e} \quad e^{-z_{b^{\prime}}}=e^{+z_{b}}\left(1+e^{+z_{e}}\right) \\
& e^{+z_{d^{\prime}}}=e^{+z_{d}}\left(1+e^{+z_{e}}\right) \quad z_{e^{\prime}}=-z_{e} \quad e^{+z_{c^{\prime}}}=e^{-z_{c}}\left(1+e^{-z_{e}}\right)
\end{aligned}
$$

\subsection{The representation of the mapping class group}

The mapping class group $\mathrm{MC}(\Sigma)$ of the topological surface $\Sigma$ is the group of isotopy classes of orientation-preserving diffeomorphisms of $\Sigma$. An element $\mu \in \operatorname{MC}(\Sigma)$ of the mapping class group will map a given (decorated) triangulation $\tau$ into another one, $\mu . \tau$. The fact that any two triangulations can be mapped into each other by a composition of the elementary transformations introduced in subsection 6.2 therefore leads to an embedding of the mapping class group into the Ptolemy groupoid:

$$
\Phi_{\tau}: \operatorname{MC}(\Sigma) \rightarrow \operatorname{Pt}(\Sigma), \quad \Phi_{\tau}(f) \equiv[\mu . \tau, \tau] .
$$

More precisely, $\Phi_{\tau}$ induces a homomorphism $\operatorname{MC}(\Sigma) \rightarrow \operatorname{Pt}(\Sigma)$ in the sense that

$$
\Phi_{\tau}\left(\mu_{2} \circ \mu_{1}\right)=\Phi_{\mu_{1} \cdot \tau}\left(\mu_{2}\right) \circ \Phi_{\tau}\left(\mu_{1}\right) \text { for any } \mu_{2}, \mu_{1} \in \operatorname{MC}(\Sigma)
$$

which embeds $\mathrm{MC}(\Sigma)$ injectively into $\operatorname{Pt}(\Sigma)[\mathrm{P} 3$, Theorem 1.3].

\section{Teichmüller space as the phase space of a constrained system}

As a preparation for the description of the quantum Teichm uller spaces it will be useful to parameterize the Teichm uller spaces by means of variables assigned to the triangles instead of the edges of a triangulation [Ka1]. In the following section we shall elaborate upon the results and constructions in [Ka1], strengthening them somewhat.

\subsection{Kashaev's coordinates}

Assume given a fat graph $\varphi$ with set of vertices $\varphi_{\mathrm{o}}$. For each vertex $v \in \varphi_{\mathrm{o}}$ one may introduce a pair of variables $\left(q_{v}, p_{v}\right)$ according to the following rule. Let us label the edges that emanate from the vertex $v$ by $e_{i}^{v}, i=1,2,3$ according to Figure 3 . We will denote the Penner coordinates associated to the edges $e_{i}^{v}$ by $l_{i}^{v}, i=1,2,3$. Let us then define the pair of variables $\left(q_{v}, p_{v}\right)$ as

$$
\left(q_{v}, p_{v}\right)=\left(l_{3}^{v}-l_{2}^{v}, l_{1}^{v}-l_{2}^{v}\right)
$$

Following Kashaev [Ka1] we will consider the vector space $V_{\varphi} \simeq \mathbb{R}^{4 M}$ obtained by regarding the variables $q_{v}, p_{v}$ as the components $q_{v}(\mathfrak{v}), p_{v}(\mathfrak{v})$ of vectors $\mathfrak{v} \in V_{\varphi}$. The space of linear coordinate functions on $V_{\varphi}$ will be called the Kashaev space $W_{\varphi}$. On $W_{\varphi}$ we will consider the Poisson bracket $\Omega_{\varphi}$ defined by

$$
\Omega_{\varphi}\left(p_{v}, q_{w}\right)=\delta_{v w}, \quad \Omega_{\varphi}\left(q_{v}, q_{w}\right)=0, \quad \Omega_{\varphi}\left(p_{v}, p_{w}\right)=0 .
$$

The assignment (7.1) associates a vector $\mathfrak{v}(P)$ in a subspace $T_{\varphi} \subset V_{\varphi}$ to each point $P \in \widetilde{\mathcal{T}}(\Sigma)$. Kashaev has observed that the subspace $T_{\varphi}$ can be characterized by a suitable set of linear forms $h_{c} \in W_{\varphi}$ (“constraints"). 


\section{The constraints}

To define the linear forms $h_{c}$ let us introduce an embedding of the first homology $H_{1}(\Sigma, \mathbb{R})$ into $W_{\varphi}$ as follows. Each graph geodesic $g_{c}$ which represents an element $[c] \in H_{1}(\Sigma, \mathbb{R})$ may be described by an ordered sequence of vertices $v_{i} \in \varphi_{\mathrm{o}}$, and edges $e_{i} \in \varphi_{1}, i=0, \ldots, n$, where $v_{0}=v_{n}, e_{0}=e_{n}$, and we assume that $v_{i-1}, v_{i}$ are connected by the single edge $e_{i}$. We will define $\omega_{i}=1$ if the arcs connecting $e_{i}$ and $e_{i+1}$ turn around the vertex $v_{i}$ in the counterclockwise sense, $\omega_{i}=-1$ otherwise. The edges emanating from $v_{i}$ will be numbered $e_{j}^{i}, j=1,2,3$ according to the convention introduced in Figure 3. To each $[c] \in H_{1}(\Sigma, \mathbb{R})$ we will assign

$$
h_{c} \equiv \sum_{i=1}^{n} u_{i}, \quad u_{i}:=\omega_{i}\left\{\begin{array}{cl}
-q_{v_{i}} & \text { if }\left\{e_{i}, e_{i+1}\right\}=\left\{e_{3}^{i}, e_{1}^{i}\right\}, \\
p_{v_{i}} & \text { if }\left\{e_{i}, e_{i+1}\right\}=\left\{e_{2}^{i}, e_{3}^{i}\right\}, \\
q_{v_{i}}-p_{v_{i}} & \text { if }\left\{e_{i}, e_{i+1}\right\}=\left\{e_{1}^{i}, e_{2}^{i}\right\} .
\end{array}\right.
$$

$h_{c}$ is independent of the choice of representative $c$ within the class $[c]$. Let $C_{\varphi}$ be the subspace in $W_{\varphi}$ that is spanned by the $h_{c},[c] \in H_{1}(\Sigma, \mathbb{R})$.

Lemma 2. - $[\mathrm{Kal}]-$

(i) The mapping $H_{1}(\Sigma, \mathbb{R}) \ni[c] \mapsto h_{c} \in C_{\varphi}$ is an isomorphism of vector spaces.

(ii) The restriction of $\Omega_{\varphi}$ to $C_{\varphi}$ coincides with the intersection form $\mathrm{I}$ on $H_{1}(\Sigma, \mathbb{R})$,

$$
\Omega_{\varphi}\left(h_{c_{1}}, h_{c_{2}}\right)=\mathrm{I}\left(c_{1}, c_{2}\right)
$$

(iii) The linear forms $h_{c},[c] \in H_{1}(\Sigma, \mathbb{R})$ vanish identically on the subspace $T_{\varphi}$.

The equations $h_{c}(\mathfrak{v})=0,[c] \in H_{1}(\Sigma, \mathbb{R})$ characterize the image of $\widetilde{\mathcal{T}}(\Sigma)$ within $V_{\varphi}$. It is useful to recall that $H_{1}(\Sigma, \mathbb{R})$ splits as $H_{1}(\Sigma, \mathbb{R})=H_{1}\left(\Sigma_{\mathrm{cl}}, \mathbb{R}\right) \oplus B(\Sigma)$, where $B(\Sigma)$ is the $s-1$-dimensional subspace spanned by the homology classes associated to the punctures of $\Sigma$, and $\Sigma_{\mathrm{cl}}$ is the compact Riemann surface which is obtained by "filling" the punctures of $\Sigma$. The corresponding splitting of $C_{\varphi}$ will be written as $C_{\varphi}=H_{\varphi} \oplus B_{\varphi}$.

\section{Change of fat graph}

In order to describe the change of Kashaev variables induced by a change of fat graph let us, following [Ka1], define the following two transformations associated to the elementary moves $\omega_{v w}$ and $\rho_{v}$ respectively.

$$
\begin{aligned}
& A_{v}:\left(q_{v}, p_{v}\right) \mapsto\left(p_{v}-q_{v},-q_{v}\right), \\
& T_{v w}:\left\{\begin{aligned}
\left(U_{v}, V_{v}\right) & \mapsto\left(U_{v} U_{w}, U_{v} V_{w}+V_{v}\right), \\
\left(U_{w}, V_{w}\right) & \mapsto\left(U_{w} V_{v}\left(U_{v} V_{w}+V_{v}\right)^{-1}, V_{w}\left(U_{v} V_{w}+V_{v}\right)^{-1}\right),
\end{aligned}\right.
\end{aligned}
$$

where we have set $U_{v} \equiv e^{q_{v}}$ and $V_{v} \equiv e^{p_{v}}$ for all $v \in \varphi_{\mathrm{o}}$.

Lemma 3. The maps $A_{v}: W_{\varphi} \rightarrow W_{\rho_{v} \circ \varphi}$ and $T_{v w}: W_{\varphi} \rightarrow W_{\omega_{v w} \circ \varphi}$ defined in (7.4) and (7.5) respectively are canonical, i.e. they preserve the Poisson structure $\Omega_{\varphi}$.

The proof is again straightforward. Lemma 3 implies in particular that the mapping class group acts on $W_{\varphi}$ by canonical transformations. 


\subsection{The structure of the Kashaev space $W_{\varphi}$}

Fock variables vs. Kashaev's variables

There is a canonical way to reconstruct the Fock-variables in terms of Kashaev's variables which is found by combining equations (5.2) and (7.1). The result may be formulated as follows. Let $v, w \in \varphi_{\mathrm{o}}$ be the vertices that are connected by the edge $e \in \varphi_{1}$, and let $e_{i}^{v}, i=1,2,3$ be the edges introduced in Figure 3.

$$
\hat{z}_{e}=\hat{z}_{e, v}+\hat{z}_{e, w}, \quad \hat{z}_{e, v}=\left\{\begin{aligned}
p_{v} & \text { if } e=e_{1}^{v}, \\
-q_{v} & \text { if } e=e_{2}^{v}, \\
q_{v}-p_{v} & \text { if } e=e_{3}^{v} .
\end{aligned}\right.
$$

The definition (7.6) defines a linear map $I_{\varphi}: F_{\varphi} \rightarrow \widehat{F}_{\varphi} \subset W_{\varphi}$. It will be useful to describe the properties of this map a bit more precisely.

\section{Lemma 4.}

$$
\begin{aligned}
\text { (i) } & \hat{z}_{e}(\mathfrak{v}(P))=z_{e}(P) \quad \forall e \in \varphi_{\mathbf{1}}, \forall P \in \widetilde{\mathcal{T}}(\Sigma), \\
\text { (ii) } & \Omega_{\varphi}\left(\hat{z}_{e}, \hat{z}_{f}\right)=\Omega_{\mathrm{WP}}\left(z_{e}, z_{f}\right) \quad \forall e, f \in \varphi_{\mathbf{1}}, \\
\text { (iii) } & \Omega_{\varphi}\left(\hat{z}_{e}, h_{c}\right)=0 \quad \forall e \in \varphi_{\mathbf{1}}, \forall c \in H_{1}(\Sigma, \mathbb{R}), \\
\text { (iv) } & \hat{f}_{c} \equiv I_{\varphi}\left(f_{c}\right)=h_{c}, \quad \forall[c] \in B(\Sigma) .
\end{aligned}
$$

Proof. Straightforward verifications.

It is also useful to remark that the transformation of the Fock variables $\hat{z}_{e}, e \in \varphi_{\mathrm{I}}$ that is induced by (7.4), (7.5) coincides with (6.3).

Splitting of $W_{\varphi}$

The linear forms $h_{c} \in B_{\varphi}$ turn out to be the Hamiltonian generators for the symmetry (5.1) [Ka1]. It is therefore natural to consider the subspace $M_{\varphi} \subset W_{\varphi}$ which is spanned by the Hamiltonian vector fields that are generated by the linear forms $h_{c} \in B_{\varphi}$, as well as $N_{\varphi} \equiv$ $M_{\varphi} \oplus B_{\varphi}$

Proposition 2. There exists a canonical transformation establishing the isomorphism of Poisson vector spaces $W_{\varphi} \simeq T_{\varphi} \oplus N_{\varphi} \oplus H_{\varphi}$, such that

(i) $T_{\varphi} \simeq \mathcal{T}^{\prime}(\Sigma)$ is the space of linear functions on the Teichmüller space $\mathcal{T}(\Sigma)$,

(ii) The restriction of $\Omega_{\varphi}$ to $T_{\varphi}$ coincides with the Poisson bracket induced by the WeilPetersson symplectic form.

Proof. As a warmup it may be instructive to count dimensions: We have $\operatorname{dim}\left(W_{\varphi}\right)=8 g-8+4 s$ and $\operatorname{dim}\left(C_{\varphi}\right)=\operatorname{dim}\left(H_{1}(\Sigma, \mathbb{R})\right)=2 g+s-1$. In order to determine $\operatorname{dim}\left(N_{\varphi}\right)$ let us choose a canonical basis for $H_{1}(\Sigma, \mathbb{R})$, represented by curves $\alpha_{1}, \ldots, \alpha_{g}, \beta_{1}, \ldots, \beta_{g}, \gamma_{1}, \ldots, \gamma_{s-1}$ such that the only nontrivial intersection pairings are $\mathrm{I}\left(\alpha_{i}, \beta_{j}\right)=\delta_{i j} . N_{\varphi}$ is spanned by the images 
of the classes $\left[\gamma_{1}\right], \ldots,\left[\gamma_{s-1}\right]$, together with the Hamiltonian vector fields that they generate. It follows that $\operatorname{dim}\left(N_{\varphi}\right)=2 s-2$.

The main point that remains to be demonstrated is the existence of a decomposition of $\widehat{F}_{\varphi}$ as the direct sum

$$
\widehat{F}_{\varphi}=T_{\varphi} \oplus B_{\varphi} \quad \text { such that } \quad \Omega_{\varphi}(t, h)=0 \quad \forall t \in T_{\varphi}, \quad \forall h \in N_{\varphi} .
$$

Thanks to Lemma 3 we may choose a convenient fat graph to this aim. Let us pick a basis $\mathfrak{B}(\Sigma)$ for $B(\Sigma)$ represented by the curves which encircle $s-1$ of the $s$ punctures. It is easy to see that we can always construct a fat graph $\varphi^{\prime}$ such that the elements of $\mathfrak{B}(\Sigma)$ are represented by singles edges in $\varphi_{1}^{\prime}$. These edges end in a unique vertex $v(c)$ for each $c \in \mathfrak{B}(\Sigma)$. It is clear that the expression for $h_{c}$ only involves the variables $\left(q_{v(c)}, p_{v(c)}\right)$ for all $c \in \mathfrak{B}(\Sigma)$. It follows from (7.2) that the Hamiltonian vector field generated by $h_{c}$ can likewise be expressed in terms of $\left(q_{v(c)}, p_{v(c)}\right)$ only. The existence of the sought-for decomposition (7.7) is obvious in this case.

The result is carried over to the general case with the help of Lemma 3. It is clear that the subspace $T_{\varphi} \subset \widehat{F}_{\varphi}$ is defined uniquely by the condition (7.7). $T_{\varphi}$ may then also be described as the quotient of $\widehat{F}_{\varphi}$ by the conditions $h_{c}=0$ for all $[c] \in B(\Sigma)$. It therefore follows from our discussion in $\S 5.3$ that $T_{\varphi}$ is canonically isomorphic to $\mathcal{T}^{\prime}(\Sigma)$, the vector space of linear functions on $\mathcal{T}(\Sigma)$.

To complete the proof it remains to observe that we have

(i) $\Omega_{\varphi}(t, h)=0 \quad \forall t \in T_{\varphi}, \quad \forall h \in H_{\varphi}$,

(ii) $\Omega_{\varphi}(h, n)=0 \quad \forall h \in H_{\varphi}, \quad \forall n \in N_{\varphi}$.

(i) follows directly from Lemma 4, whereas part (ii) can easily be verified by considering the fat graph $\varphi^{\prime}$ above.

\section{The Fenchel-Nielsen coordinates}

We will now be interested in the case of Riemann surfaces with a boundary that is represented by a collection of $s>0$ geodesics. Another standard set of coordinates for the Teichm uller spaces is associated to the decomposition of a Riemann surface into trinions (three-holed spheres). We are now going to review the definition of these coordinates. Different sets of Fenchel-Nielsen coordinates will be associated to different markings of the Riemann surface in a way which is analogous to the relation between the Penner coordinates and fat graphs.

Let us denote by $S_{3}$ the sphere with three holes (trinion). As a concrete model we may e.g. choose

$$
S_{3} \equiv\{z \in \mathbb{C} ;|z| \geq \epsilon,|1-z| \geq \epsilon,|z|<1 / \epsilon\}
$$

Any trinion with a smooth boundary is diffeomorphic to $S_{3}$.

Definition 1. A marking $\sigma$ of a surface $\Sigma$ consists of the following data.

(i) A cut system $\mathcal{C}_{\sigma}$, which is a set $\mathcal{C}_{\sigma}=\left\{c_{1}, \ldots, c_{3 g-3+s}\right\}$ of simple non-intersecting oriented closed curves $c_{i}$ on $\Sigma$. Cutting $\Sigma$ along the curves in $\mathcal{C}$ decomposes the surface into a collection $\mathcal{P}_{\sigma}$ of trinions. 
(ii) A choice of a trivalent graph $\Gamma_{T}$ with a single vertex $v_{T}$ in each trinion $T \in \mathcal{P}_{\sigma}$ such that the graphs on the different trinions glue to a connected graph $\Gamma_{\sigma}$ on $\Sigma$.

(iii) A choice of a distinguished boundary component for each trinion $T \in \mathcal{P}_{\sigma}$.

These data will be considered up to isotopy.

An example for the graphs $\Gamma_{T}$ is depicted in Figure 10.

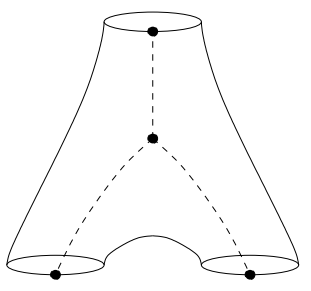

Figure 8: A trinion equipped with a marking graph

\subsection{Definition of the Fenchel-Nielsen coordinates}

The basic observation underlying the definition of the Fenchel-Nielsen twist coordinates is the fact that for each triple $\left(l_{1}, l_{2}, l_{3}\right)$ of positive real numbers there is a unique metric of constant curvature -1 on the three-holed sphere (trinion) such that the boundary components are geodesics with lengths $l_{i}, i=1,2,3$. A trinion with its metric of constant curvature -1 will be called hyperbolic trinion. There furthermore exist three distinguished geodesics on each hyperbolic trinion that connect the boundary components pairwise.

Let us assume that the geodesic $c$ separates two trinions $T_{a}$ and $T_{b}$. Pick boundary components $c_{a}$ and $c_{b}$ of $T_{a}$ and $T_{b}$ respectively by starting at $c$, following the marking graphs, and turning left at the vertices. As mentioned above, there exist distinguished geodesics on $T_{a}$ and $T_{b}$ that connect $c$ with $c_{a}$ and $c_{b}$ respectively. Let $\delta_{c}$ be the signed geodesic distance between the end-points of these geodesics on $c$, and let

$$
\theta_{c}=2 \pi \frac{\delta_{c}}{l_{c}}
$$

be the corresponding twist-angle. In a similar way one may define $\theta$ in the case that cutting along $c$ opens a handle.

Given a cut system $\mathcal{C}=\left\{c_{1}, \ldots, c_{\kappa}\right\}, \kappa=3 g-3+s$, we thereby associate to each Riemann surface $\Sigma$ a tuple $\left(l_{1}, \ldots, l_{\kappa} ; \theta_{1}, \ldots, \theta_{\kappa}\right)$ of real numbers. It can be shown (see e.g. [IT]) that the Riemann surface $\Sigma$ is characterized uniquely by the tuple $\left(l_{1}, \ldots, l_{\kappa} ; e^{i \theta_{1}}, \ldots, e^{i \theta_{\kappa}}\right) \in\left(\mathbb{R}^{+}\right)^{\kappa} \times$ $\left(S^{1}\right)^{\kappa}$. In order to describe the Teichm uller space $\mathcal{T}(\Sigma)$ of deformations of $\Sigma$ it suffices to allow for arbitrary real values of the twist angles $\theta_{i}$. Points in $\mathcal{T}(\Sigma)$ are then parametrized by tuples $\left(l_{1}, \ldots, l_{\kappa} ; \theta_{1}, \ldots, \theta_{\kappa}\right) \in\left(\mathbb{R}^{+}\right)^{\kappa} \times \mathbb{R}^{\kappa}$.

Remark 2. The marking graph $\Gamma_{\sigma}$ allows one to distinguish systems of Fenchel-Nielsen coordinates which are related to each other by Dehn twists, $\theta_{c}^{\prime}=\theta_{c}+2 \pi k_{c}, k \in \mathbb{Z}, c \in \mathcal{C}$. To use the 
markings for the parametrization of different systems of Fenchel-Nielsen coordinates is then closely analogous to using fat graphs for the specification of systems of Penner coordinates.

The definition of the Fenchel-Nielsen coordinates does not use the choice of a distinguished boundary component for each trinion. The latter has been included into the definition 1 for later convenience only.

\subsection{Symplectic structure}

Let us furthermore notice that the Weil-Petersson symplectic form becomes particularly simple in terms of the Fenchel-Nielsen coordinates:

Theorem 3. [Wo2] -

$$
\omega_{\mathrm{WP}}=\sum_{i=1}^{\kappa} d \tau_{i} \wedge d l_{i}, \quad \tau_{i}=\frac{1}{2 \pi} l_{i} \theta_{i} .
$$

The content of the theorem may also be paraphrased as follows:

(i) The geodesic length functions associated to non-intersecting closed curves Poissoncommute.

(ii) The Hamiltonian flows generated by the geodesic length functions coincide with the Fenchel-Nielsen twist flows.

\subsection{Geodesic lengths from the Penner coordinates}

A nice feature of the Fock coordinates is that they lead to a particularly simple way to reconstruct the Fuchsian group corresponding to the point $P$ in Teichm uller space that is parametrized by the variables $z_{e}(P), e \in \varphi_{\mathrm{I}}$. Assume given a graph geodesic $g_{c}$ on the fat graph homotopic to a simple closed curve $c$ on $\Sigma$. Let the edges be labelled $e_{i}, i=1, \ldots, r$ according to the order in which they appear on $g_{c}$, and define $\sigma_{i}$ to be 1 if the path turns left ${ }^{5}$ at the vertex that connects edges $e_{i}$ and $e_{i+1}$, and to be equal to -1 otherwise. The generator $\mathrm{X}(c)$ of the Fuchsian group that corresponds to $c$ is then constructed as follows [Fo].

$$
\mathrm{X}_{c}=\mathrm{V}^{\sigma_{r}} \mathrm{E}\left(z_{e_{r}}\right) \ldots \mathrm{V}^{\sigma_{1}} \mathrm{E}\left(z_{e_{1}}\right),
$$

where the matrices $\mathrm{E}(z)$ and $\mathrm{V}$ are defined respectively by

$$
\mathrm{E}(z)=\left(\begin{array}{cc}
0 & +e^{+\frac{z}{2}} \\
-e^{-\frac{z}{2}} & 0
\end{array}\right), \quad V=\left(\begin{array}{rr}
1 & 1 \\
-1 & 0
\end{array}\right) .
$$

Given the generator $\mathrm{X}_{c}$ of the Fuchsian group one may then calculate the hyperbolic length of the closed geodesic isotopic to $c$ via

$$
2 \cosh \left(\frac{1}{2} l_{c}\right)=\left|\operatorname{tr}\left(\mathrm{X}_{c}\right)\right|
$$

The proof of (8.4) was omitted in [Fo]. We are therefore now going to explain how to verify the validity of equation (8.4).

\footnotetext{
${ }^{5}$ w.r.t. to the orientation induced by the embedding of the fat-graph into the surface
} 
It was remarked in the Subsection $\S 5.3$ that for given values of the coordinates $z_{e}$ one may construct a uniformized representation of the corresponding Riemann surface by successively mapping ideal hyperbolic triangles into the upper half plane which are glued according to the values $z_{e}$. Iterating this procedure ad infinitum one generates a tessellation of the upper half plane by ideal hyperbolic triangles. Let us now consider a generator $\mathrm{X}(c)$ of the Fuchsian group, represented on the upper half plane by a M obius transformation $M_{\mathrm{X}(c)}$, where $M_{\mathrm{X}}(u) \equiv$ $\frac{a u+b}{c u+d}$ if $X=\left(\begin{array}{ll}a & b \\ c & d\end{array}\right)$. The element $c \in \pi_{1}(\Sigma)$ may then be represented by an open path on the upper half plane which leads from a chosen base point $u$ to its image under $M_{\mathrm{X}}$.

But one may equivalently represent the motion along the path by standing still at the base point and moving the tessellation around by means of $\mathrm{M}$ ”obius-transformations. More precisely, let us assume that our base point $u$ is located within the ideal hyperbolic triangle $t_{0}$ with corners at $-1,0, \infty$, and that the path $\mathcal{P}_{c}$ representing our chosen element $c \in \pi_{1}(\Sigma)$ crosses the edges $e_{i} i=1, \ldots, r$ in the order of the labelling. We may assume that the edge $e_{1}$ connects the points 0 and $e^{z_{e_{1}}}$. After having crossed edge $e_{1}$ one would have left the triangle $t_{0}$ into the triangle $t_{1}$ with corners at $0, e^{z_{e_{1}}}, \infty$. The Möobius-transformation $M_{1}$ corresponding to $\mathrm{E}\left(z_{e_{1}}\right)$ brings one back into $t_{0}$ : It can be checked that it leaves the set of corners $\left\{-1,0, e^{z_{e_{1}}}, \infty\right\}$ on the two triangles glued along $e_{1}$ invariant, but exchanges the two triangles. To continue along the path $\mathcal{P}_{c}$ in this fashion we now need to map the next edge $e_{2}$ to be crossed to the edge going from 0 to $\infty$ before we can apply the $\mathrm{M}$ "obius-transformation corresponding to $\mathrm{E}\left(z_{e_{2}}\right)$ in the same manner as before. This is precisely what the Mobius-transformation $M_{\sigma^{\sigma_{1}}}$ does: It simply rotates the edges of our fundamental triangle $t_{0}$. Moreover, the triangle $t_{2}$ that would be reached when leaving $t_{0}$ through $e_{2}$ will be mapped by $M_{\mathrm{V}^{\sigma_{1}}}$ into the ideal hyperbolic triangle with corners $0, e^{z_{e_{2}}}, \infty$. The fact that $e^{z_{e_{2}}}$ is indeed the position that the corner of $t_{2}$ is mapped into by $M_{\mathrm{V}^{\sigma_{1}}}$ follows from the prescription for gluing $t_{1}$ and $t_{2}$ along $e_{2}$ in terms of $z_{e_{2}}$ and the fact that $M_{\mathrm{V}^{\sigma_{1}}}$ preserves cross-ratios.

By continuing in this fashion one generates the M"obius transformation $M_{\mathrm{X}(c)}$ that evidently maps the original tessellation representing the chosen point $P$ in $\mathcal{T}(\Sigma)$ into another one that is equally good as a representation for $P$. By assumption, $M_{\mathrm{X}(c)}$ represents a closed path on the considered fat graph. This means that the points that are mapped into each other by $M_{\mathrm{X}(c)}$ are to be identified as different representatives for the same points on the surface corresponding to our point $P \in \mathcal{T}(\Sigma)$.

\section{Coordinates for surfaces with holes of fi nite size}

In the present paper we are mainly interested in the case of Riemann surfaces which have a boundary $\partial \Sigma$ represented by $s$ geodesics of finite length. We therefore need to discuss how to introduce analogs of the previously described coordinate systems for $\mathcal{T}(\Sigma)$ for the cases of interest here. When considering surfaces with holes of finite size one has to choose if one wants to keep the geodesic lengths of the boundary components variable, or if one wants to consider surfaces $\Sigma_{\Lambda}$ which have fixed boundary length given by the tuple $\Lambda=\left(l_{1}, \ldots, l_{s}\right) \in \mathbb{R}_{+}^{s}$. We shall find the first option often more convenient to work with. Passing to a representation in which the boundary lengths are fixed will then be almost trivial. 


\subsection{Useful fat graphs on surfaces with holes of finite size}

Riemann surfaces $\Sigma$ with $s$ holes can always be represented by considering a Riemann surface $\Sigma^{\mathrm{e}}$ with $s$ pairs of punctures, from which $\Sigma$ is obtained by cutting $\Sigma^{\mathrm{e}}$ along the geodesics $b_{1}, \ldots, b_{s}$ that encircle the pairs of punctures. This simple observation allows us to use the coordinates discussed previously in order to define coordinates for the Teichm uller spaces of surfaces with $s$ holes. In order to spell out more precisely how to do this, let us first introduce a convenient class of fat graphs.

Let us consider a pair of punctures $\left(P_{1}, P_{2}\right)$. Let $c$ be a geodesic such that cutting $\Sigma$ along $c$ produces two connected components one of which is a two-punctured disc $D$ with punctures $P_{1}$ and $P_{2}$. A given fat graph $\varphi^{\mathrm{e}}$ will be said to have standard form near $D$ if there exists a neighborhood of the disc $D$ in which $\varphi^{\mathrm{e}}$ is homotopic to the fat graph depicted on the left half of Figure 9.
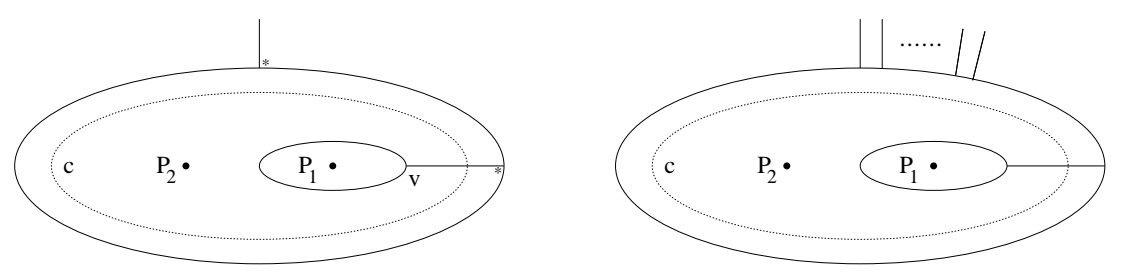

Figure 9: Simple fat graphs in a neighborhood of the disc defined by the geodesic $c$ which encircles two punctures $P_{1}$ and $P_{2}$.

For surfaces $\Sigma^{\mathrm{e}}$ with $s$ pairs of punctures there exist fat graphs $\varphi^{\mathrm{e}}$ which are of standard form in the neighborhood of $s-1$ discs $D_{i}$. The simplest possible form of a fat graph around the remaining two punctures is indicated on the right of Figure 9. A fat graph $\varphi^{\mathrm{e}}$ on a surface with $2 s$ punctures will be said to have standard form if it has standard form near $s-1 \operatorname{discs} D_{i}$, and if it has the form depicted on the right of Figure 9 in a neighborhood of the remaining disc.

We will finally say that a fat graph $\varphi$ on a Riemann surface $\Sigma$ with $s$ geodesic boundaries has standard form if $(\Sigma, \varphi)$ can be obtained from a pair $\left(\Sigma^{\mathrm{e}}, \varphi^{\mathrm{e}}\right)$ consisting of a $2 s$-punctured surface $\Sigma$ and a fat graph $\varphi^{\mathrm{e}}$ of standard form by cutting $\Sigma^{\mathrm{e}}$ along $s$ geodesics $b_{1}, \ldots, b_{s}$, each of which encircles a pair of punctures. The embedding $\Sigma \hookrightarrow \Sigma^{\mathrm{e}}$ furthermore induces an embedding $\operatorname{MC}(\Sigma) \hookrightarrow \operatorname{MC}\left(\Sigma^{\mathrm{e}}\right)$ of the respective mapping class groups. The subgroup of $\mathrm{MC}\left(\Sigma^{\mathrm{e}}\right)$ which is generated by the diffeomorphisms that are supported on $\Sigma \subset \Sigma^{\mathrm{e}}$ preserves the set of fat graphs which have standard form.

\subsection{Kashaev type coordinates}

If we only use fat graphs of standard form, it becomes easy to adapt the previously discussed systems of coordinates to the case of interest in the rest of this paper. We may in particular consider the space $W_{\varphi}$ of Kashaev variables associated to the fat graph $\varphi$. A subspace $T_{\varphi}$ of $W_{\varphi}$ can again be defined by means of the decomposition (7.7). It is furthermore convenient to introduce the set $\varphi_{1}^{\prime}$ which only contains the edges of $\varphi$ that do not end in boundary components of $\Sigma$.

Lemma 5. (i) We have $T_{\varphi} \simeq T_{\varphi^{\mathrm{e}}}$. 
(ii) The Fock coordinates $\left\{z_{e} ; e \in \varphi_{1}^{\prime}\right\}$ form a set of coordinates for $T_{\varphi}$.

Proof. Let us first note that the linear form $h_{1}$ which is via (7.3) associated to puncture $P_{1}$ can be expressed in terms of the variables $\left(p_{v}, q_{v}\right)$ associated to the vertex $v$ in Figure 9 only. This means that both $p_{v}$ and $q_{v}$ are contained in $N_{\varphi^{\mathrm{e}}}$. Instead of the linear form $h_{2}$ associated to puncture $P_{2}$ we may consider $h_{c}=h_{1}+h_{2}$, which can be expressed exclusively in terms of the variables associated to the vertices contained in $\Sigma$. Part (i) of the lemma follows easily from these observations.

In order to verify part (ii) one may again consider $\varphi^{\mathrm{e}}$. When writing the relations $f_{c}=0$, $c \in B(\Sigma)$ in terms of the Fock variables $z_{e}, e \in \varphi_{1}^{\mathrm{e}}$ one will always find contributions containing the $z_{e}, e \in \varphi_{1}^{\mathrm{e}} \backslash \varphi_{1}^{\prime}$. It is then easy to convince oneself that the relations $\hat{f}_{c}=0$ may be used to express the $z_{e}, e \in \varphi_{1}^{\mathrm{e}} \backslash \varphi_{1}^{\prime}$ in terms of the $z_{e}, e \in \varphi_{1}^{\prime}$. After this is done, all relations $f_{c}=0$, $c \in B(\Sigma)$ are satisfied, the variables $z_{e}, e \in \varphi_{1}^{\prime}$ are therefore unconstrained.

However, in this case the relation between the Teichm uller space $\mathcal{T}(\Sigma)$ and $T_{\varphi}$ is slightly more complicated. In order to describe this relation let us consider the spaces $\operatorname{Fun}(\mathcal{T}(\Sigma))$ and $\operatorname{Fun}\left(T_{\varphi}\right)$ of smooth functions on $\mathcal{T}(\Sigma)$ and $T_{\varphi}$ respectively. These spaces carry canonical Poisson brackets $\{., .\}_{\mathrm{WP}}$ and $\{., .\}_{\varphi}$ uniquely defined by the bilinear forms $\Omega_{\mathrm{WP}}$ and $\Omega_{\varphi}$ respectively.

Proposition 3. We may represent $\operatorname{Fun}(\mathcal{T}(\Sigma))$ as the subspace of $\operatorname{Fun}\left(T_{\varphi}\right)$ which is defined by the conditions

$$
\left\{F, l_{i}\right\}_{\varphi}=0, \quad i=1, \ldots, s, \quad F \in \operatorname{Fun}\left(T_{\varphi}\right),
$$

where $l_{i}$ is the length function which is associated to the $i$-th boundary component via equations (8.4) and (8.6).

It should be noted that the length functions associated to the boundary components are contained in $\operatorname{Fun}(\mathcal{T}(\Sigma))$. 


\section{Part III}

\section{Quantization of the Teichmüller spaces}

Our aim is to construct certain classes of infinite-dimensional representations of the mapping class groups $\mathrm{MC}(\Sigma)$. One possible approach to this problem is to "quantize" Poisson manifolds like the Teichm uller spaces on which $\mathrm{MC}(\Sigma)$ acts as a group of symmetries.

Our construction will proceed in two main steps. Quantization of the Kashaev spaces $W_{\varphi}$ leads to a rather elegant construction of projective unitary representations of the mapping class groups [Ka1]. However, these representations turn out to be reducible. The second step will therefore be to identify distinguished subrepresentations within the representations coming from the quantization of the Kashaev spaces as the mapping class group representations which are naturally associated to the quantization of the Teichm uller spaces. A direct construction of the latter is not known, which is why this somewhat indirect construction seems to be most efficient at the moment.

\section{Quantization of the Teichmüller spaces}

\subsection{Canonical quantization}

Quantization of a Poisson manifold $\mathcal{P}$ means "deforming" the space of functions on $\mathcal{P}$ into a one-parameter $(\hbar)$ family of noncommutative algebras $\mathcal{P}_{\hbar}$ in such a way that the deformed product $f *_{\hbar} g$ satisfies

$$
f *_{\hbar} g=f g+\hbar \Omega(f, g)+\mathcal{O}\left(\hbar^{2}\right)
$$

where $f g$ is the ordinary commutative product of functions on $\mathcal{P}$ and $\Omega(f, g)$ is the Poisson bracket on $\mathcal{P}$. If the Poisson manifold $\mathcal{P}$ has a group $G$ of symmetries it is natural to demand that these symmetries are preserved by quantization in the sense that any $g \in G$ is realized as an automorphism $f \rightarrow \mathrm{a}_{g}(f)$ of $\mathcal{P}_{\hbar}$.

Representations of the group $G$ can be constructed by studying representations of the algebra $\mathcal{P}_{\hbar}$ by operators $\mathrm{O}(f)$ on a Hilbert space $\mathcal{H}$,

$$
\mathrm{O}(f) \cdot \mathrm{O}(g)=\mathrm{O}\left(f *_{\hbar} g\right) .
$$

The Hilbert space $\mathcal{H}$ will then typically come equipped with a unitary projective representation of the group $G$ of symmetries by operators $\mathrm{U}_{g}$ such that the automorphisms $\mathrm{A}_{g}(\mathrm{O}(f)) \equiv$ $\mathrm{O}\left(\mathrm{a}_{g}(f)\right)$ are realized as

$$
\mathrm{A}_{g}(\mathrm{O})=\mathrm{U}_{g} \cdot \mathrm{O} \cdot \mathrm{U}_{g}^{-1}
$$

Quantization is particularly simple if there exist coordinate functions $q_{1}, \ldots, q_{N}$ and $p_{1}, \ldots, p_{N}$ defined globally on $\mathcal{P}$ such that the Poisson bracket takes the form

$$
\Omega\left(p_{v}, q_{w}\right)=\delta_{v w}, \quad \Omega\left(q_{v}, q_{w}\right)=0, \quad \Omega\left(p_{v}, p_{w}\right)=0,
$$

for $v, w \in\{1, \ldots, N\}$. One may then define $\mathcal{P}_{\hbar}$ in such a way that the relations

$$
i\left[p_{v}, q_{w}\right]=\hbar \delta_{v w}, \quad\left[q_{v}, q_{w}\right]=0, \quad\left[p_{v}, p_{w}\right]=0,
$$


hold for $[f, g] \equiv f *_{\hbar} g-g *_{\hbar} f$. There exists a standard representation $\mathrm{O}$ of these commutation relations on (dense subspaces of) the Hilbert space $\mathcal{H}=L^{2}\left(\mathbb{R}^{N}\right)$ of square-integrable functions $\Psi(\mathfrak{q}), \mathfrak{q}=\left(q_{1}, \ldots, q_{N}\right)$, which is generated by pairs of operators

$$
\mathrm{p}_{v} \equiv \frac{1}{2 \pi \hbar} \mathrm{O}\left(p_{v}\right), \quad \mathrm{q}_{v} \equiv \mathrm{O}\left(q_{v}\right), \quad v=1, \ldots, N
$$

that are defined respectively by

$$
\mathbf{q}_{v} \Psi(\mathfrak{q}) \equiv q_{v} \Psi(\mathfrak{q}), \quad \mathbf{p}_{v} \Psi(\mathfrak{q}) \equiv \frac{1}{2 \pi i} \frac{\partial}{\partial q_{v}} \Psi(\mathfrak{q})
$$

This simple example for the quantization of Poisson manifolds is often referred to as "canonical quantization".

Remark 3. The representation that is constructed in this way is irreducible in the following sense. If $\mathrm{O}$ is a bounded operator on $L^{2}\left(\mathbb{R}^{N}\right)$ which commutes ${ }^{6}$ with the operators $\mathrm{p}_{v}$ and $\mathrm{q}_{v}$ for all $v=1, \ldots, N$ then $\mathrm{O}=\chi$, the operator of multiplication with the complex number $\chi$.

Following the discussion in Sections 5, it seems natural to define the quantized Teichm uller spaces as the noncommutative algebra $\mathcal{T}_{\hbar}(\Sigma)$ with generators $z_{e}$ and relations

(i) $\quad i\left[z_{e_{2}}, z_{e_{1}}\right]=\hbar \Omega_{\mathrm{wP}}\left(z_{e_{2}}, z_{e_{1}}\right)$

(ii) $\quad f_{\varphi, c}=0, \forall c \in B(\Sigma)$.

The space $\mathcal{H}(\Sigma)$ will be defined as an irreducible representation of the commutation relations (10.6) which satisfies the additional conditions (10.7).

\subsection{Quantization of the Kashaev space $W_{\varphi}$}

For each given fat graph $\varphi$ let us define the Hilbert space $\mathcal{K}(\varphi)$ as the space of square integrable functions $\Psi(\mathfrak{q})$ of the Kashaev-variables $\mathfrak{q}=\left(q_{1}, \ldots, q_{2 M}\right)$. On $\mathcal{K}(\varphi)$ we shall consider the basic operators $\mathrm{p}_{v}, \mathrm{q}_{v}$ defined in (10.5) for $v=1, \ldots, 2 M$. The noncommutative algebras of operators which are generated by the operators $\mathrm{q}_{v}, \mathrm{p}_{w}$ with the commutation relations

$$
\left[\mathrm{p}_{v}, \mathrm{q}_{w}\right]=(2 \pi i)^{-1} \delta_{v, w}
$$

may be considered as representing quantized algebras of functions on the Kashaev space $W_{\varphi}$.

Remark 4. It is worth noting that the decoration of the triangles is used to define the concrete realization of the space $\mathcal{K}(\varphi)$ as a space of square integrable functions.

When quantizing the Kashaev spaces $W_{\varphi}$ we get more than we ultimately want. In order to see this, let us introduce quantum analogs of the coordinate functions $h_{c}$ and $\hat{z}_{e}$ respectively, i.e.

\footnotetext{
${ }^{6}$ Commutativity $[\mathrm{O}, \mathrm{A}]=0$ with a self-adjoint unbounded operator $\mathrm{A}$ is, by convention, understood in the sense of commutativity with the spectral projections of A. For the reader's convenience, we have collected the relevant operator-theoretical results in Appendix B.
} 
self-adjoint operators $\hat{\mathrm{h}}_{c}$ and $\hat{\mathrm{z}}_{e}$ on $\mathcal{H}(\varphi)$ which are defined by formulae very similar to (7.3) and (7.6) respectively, normalized in such a way that the following commutation relation hold:

$\begin{array}{ll}\text { (i) } & i\left[\hat{\mathrm{z}}_{e_{1}}, \hat{\mathrm{z}}_{e_{2}}\right]=\hbar \Omega_{\mathrm{WP}}\left(z_{e_{1}}, z_{e_{2}}\right), \\ \text { (ii) } & i\left[\hat{\mathrm{h}}_{c_{1}}, \hat{\mathrm{h}}_{c_{2}}\right]=\hbar \mathrm{I}\left(c_{1}, c_{2}\right) . \\ \text { (iii) } & {\left[\hat{\mathrm{z}}_{e}, \hat{\mathrm{h}}_{c}\right]=0 \quad \forall c \in H_{1}(\Sigma, \mathbb{R}) .}\end{array}$

We observe that we $d o$ have a representation, henceforth denoted $Z_{\varphi}$, of the algebra (10.6), but this representation is neither irreducible, nor does it fulfill the additional relations (10.7). The latter point becomes most clear if one introduces the operators $\hat{\mathrm{f}}_{c}, c \in B(\Sigma)$ associated to the relations (10.7) which are defined by replacing $z_{e} \rightarrow \hat{z}_{e}$ in (5.3). We may then observe that

$$
\hat{\mathrm{f}}_{c}=\mathrm{h}_{c} \quad \text { for } c \in B(\Sigma)
$$

which is verified in the same way as statement (iv) in Lemma 4.

\subsection{Reduction to the quantized Teichmüller spaces}

In order to see that the representations $Z_{\varphi}$ "contain" irreducible representations of the quantized Teichm uller spaces $\mathcal{T}(\Sigma)$, let us consider the noncommutative algebra $\mathcal{Z}_{\hbar}(\Sigma)$ with generators $z_{e}$ and the only relations (10.6). One should observe that the $f_{c}$ generate the center of the algebra $\mathcal{Z}_{\hbar}$. Irreducible unitary representations $Z_{\mathfrak{f}}$ of this algebra are parametrized by linear functions $\mathfrak{f}: B(\Sigma) \rightarrow \mathbb{R}$. Such representations are such that the operators $\mathfrak{f}_{c}=\mathbf{Z}_{\mathfrak{f}}\left(f_{c}\right)$ are realized as the operators of multiplication with the real numbers $\mathfrak{f}(c), c \in B(\Sigma)$.

The representations can be constructed concretely by forming linear combinations $t_{k}$ and $t_{k^{\prime}}^{\vee}$, $k=1, \ldots, 3 g-3+s$ of the $z_{e}$ which

(i) are mutually linearly independent, and linearly independent of the $f_{c}$,

(ii) and which satisfy the commutation relations

$$
2 \pi\left[t_{k}, t_{k^{\prime}}^{\vee}\right]=i \delta_{k k^{\prime}}
$$

Canonical quantization realizes $Z_{\mathfrak{f}}$ on the Hilbert space $\mathcal{H}_{\mathfrak{f}}(\varphi) \simeq L^{2}\left(\mathbb{R}^{3 g-3+s}\right)$ which consists of square-integrable functions $\Phi(\mathfrak{t}), \mathfrak{t}=\left(t_{1}, \ldots, t_{3 g-3+s}\right)$. The operators $\mathbf{z}_{\mathfrak{f}, e} \equiv Z_{\mathfrak{f}}\left(z_{e}\right)$ are then realized as linear combination of the operators

$$
\mathrm{t}_{k} \Phi_{\mathfrak{f}}(\mathfrak{t})=t_{k} \Phi_{\mathfrak{f}}(\mathfrak{t}), \quad \mathrm{t}_{k}^{\vee} \Phi_{\mathfrak{f}}(\mathfrak{t})=\frac{1}{2 \pi i} \frac{\partial}{\partial t_{k}} \Phi_{\mathfrak{f}}(\mathfrak{t}), \quad \mathfrak{f}_{c} \Phi_{\mathfrak{f}}(\mathfrak{t})=\mathfrak{f}(c) \Phi_{\mathfrak{f}}(\mathfrak{t})
$$

where $k=1, \ldots, 3 g-3+s$ and $c \in B(\Sigma)$.

Our aim is to describe how the representation $Z_{\varphi}$ decomposes into the representations $Z_{\mathfrak{f}}$. In order to do this, let us introduce the representation

$$
\mathrm{Z}_{\varphi}^{\prime} \equiv \int_{B^{\prime}(\Sigma)}^{\oplus} d \mathfrak{f} Z_{\mathfrak{f}} \quad \text { on the space } \quad \mathcal{H}_{z}(\varphi) \equiv \int_{B^{\prime}(\Sigma)}^{\oplus} d \mathfrak{f} \mathcal{H}_{\mathfrak{f}}(\varphi)
$$


The space $\mathcal{H}_{z}(\varphi)$ is spanned by square-integrable families $\Phi \equiv\left(\Phi_{\mathfrak{f}}\right)_{\mathfrak{f} \in B^{\prime}(\Sigma)}$ of functions $\Phi_{\mathfrak{f}} \in$ $\mathcal{H}_{\mathfrak{f}}(\varphi)$ which are associated to the linear functions $\mathfrak{f}: B(\Sigma) \rightarrow \mathbb{R}$ in the dual $B^{\prime}(\Sigma) \simeq \mathbb{R}^{s-1}$ up to a set of measure zero. The representatives $\mathrm{z}_{e} \equiv \mathrm{Z}_{\varphi}\left(z_{e}\right)$ are defined as follows

$$
\mathrm{z}_{e} \Phi \equiv\left(\mathrm{z}_{\mathfrak{f}, e} \Phi_{\mathfrak{f}}\right)_{\mathfrak{f} \in B^{\prime}(\Sigma)} .
$$

Proposition 4. The decomposition of the representation $\mathrm{Z}_{\varphi}$ into irreducible representations of $\mathcal{Z}_{\hbar}$ may be written as follows:

$$
\mathrm{Z}_{\varphi} \simeq\left(\int_{B^{\prime}(\Sigma)}^{\oplus} d \mathfrak{f} \mathrm{Z}_{\mathfrak{f}}\right) \otimes 1_{\mathcal{H}_{h}(\varphi)}
$$

where the space $\mathcal{H}_{h}(\varphi)$ is isomorphic to $L^{2}\left(\mathbb{R}^{g}\right)$. There exists a unitary operator $\mathrm{I}_{\varphi}, \mathrm{I}_{\varphi}$ : $\mathcal{K}(\varphi) \rightarrow \mathcal{H}_{z}(\varphi) \otimes \mathcal{H}_{h}(\varphi)$ such that

$$
\mathrm{I}_{\varphi} \cdot \hat{\mathrm{z}}_{e} \cdot \mathrm{I}_{\varphi}^{-1}=\mathrm{z}_{e} \otimes 1 \quad \text { and } \quad \mathrm{I}_{\varphi} \cdot \hat{\mathrm{h}}_{c} \cdot \mathrm{I}_{\varphi}^{-1}=1 \otimes \mathrm{h}_{c},
$$

for any $e \in \varphi_{1}$ and $c \in H_{1}\left(\Sigma_{\mathrm{cl}}, \mathbb{R}\right)$, respectively.

Proof. Let us recall the direct sum decomposition

$$
W_{\varphi} \simeq T_{\varphi} \oplus N_{\varphi} \oplus H_{\varphi}
$$

To each of the three spaces $T_{\varphi}, N_{\varphi}, H_{\varphi}$ one may choose coordinates which bring the Poisson bracket to the canonical form (10.3). The corresponding operators

$$
\begin{aligned}
& \left(\hat{\mathrm{t}}_{k}, \hat{\mathrm{t}}_{k}^{\vee}\right), \quad k=1, \ldots, 3 g-3+s, \\
& \left(\hat{\mathrm{f}}_{l}, \hat{\mathrm{f}}_{l}^{\vee}\right), \quad l=1, \ldots, s-1, \\
& \left(\hat{\mathrm{h}}_{m}, \hat{\mathrm{h}}_{m}^{\vee}\right), \quad m=1, \ldots, g,
\end{aligned}
$$

can be constructed as linear combinations of the $\left(\mathrm{p}_{v}, \mathrm{q}_{v}\right), v \in \varphi_{1}$, in such a way that the only nontrivial commutation relations are

$$
\begin{aligned}
& 2 \pi\left[\hat{\mathrm{t}}_{k}, \hat{\mathrm{t}}_{k^{\prime}}^{\vee}\right]=i b^{2} \delta_{k k^{\prime}}, \quad k, k^{\prime}=1, \ldots, 3 g-3+s, \\
& 2 \pi\left[\hat{\mathrm{f}}_{l}, \hat{\mathrm{f}}_{l^{\prime}}^{\vee}\right]=i b^{2} \delta_{l l^{\prime}}, \quad l, l^{\prime}=1, \ldots, s-1, \\
& 2 \pi\left[\hat{\mathrm{h}}_{m}, \hat{\mathrm{h}}_{m^{\prime}}^{\vee}\right]=i b^{2} \delta_{m m^{\prime}}, \quad m, m^{\prime}=1, \ldots, g .
\end{aligned}
$$

It will be convenient to form the following vectors with $4 M$ operator-valued components:

$$
\begin{aligned}
& \mathrm{v}=\left(\ldots, \mathrm{q}_{v}, \ldots, \mathrm{p}_{w}, \ldots\right) \\
& \hat{\mathrm{v}}=\left(\ldots, \hat{\mathrm{t}}_{k}, \ldots, \hat{\mathrm{f}}_{l}, \ldots, \hat{\mathrm{h}}_{m}, \ldots, \hat{\mathrm{t}}_{k}^{\vee}, \ldots, \hat{\mathrm{f}}_{l}^{\vee}, \ldots, \hat{\mathrm{h}}_{m}^{\vee}, \ldots\right) .
\end{aligned}
$$

The linear change of variables $\hat{v}=\hat{v}(v)$ can then be represented by a symplectic $(4 M \times 4 M)$ matrix $J_{\varphi}$,

$$
\hat{\mathrm{v}}=J_{\varphi} \mathrm{v}, \quad J_{\varphi} \in S p(2 M, \mathbb{R}) .
$$

On the other hand let us note that $\mathcal{H}_{z}(\varphi) \otimes \mathcal{H}_{h}(\varphi)$ is canonically isomorphic to $L^{2}\left(\mathbb{R}^{2 M}\right)$ via

$$
\mathrm{K}: \Phi \otimes \psi \rightarrow \Psi, \quad \Psi(\mathfrak{t}, \mathfrak{f}, \mathfrak{h}) \equiv \Phi_{\mathfrak{f}}(\mathfrak{t}) \psi(\mathfrak{h})
$$


The corresponding representation of the commutation relations (10.18) on the Hilbert space $L^{2}\left(\mathbb{R}^{2 M}\right)$ is obtained by renaming the operators $q_{v}, p_{v}$ as follows

$$
\begin{aligned}
& \left(\ldots, \mathrm{q}_{v}, \ldots, \mathrm{p}_{w}, \ldots\right) \\
& \quad \equiv\left(\ldots, \mathrm{t}_{k}, \ldots, \mathrm{f}_{l}, \ldots, \mathrm{h}_{m}, \ldots, \mathrm{t}_{k}^{\vee}, \ldots, \mathrm{f}_{l}^{\vee}, \ldots, \mathrm{h}_{m}^{\vee}, \ldots\right) .
\end{aligned}
$$

It follows from the Stone - von Neumann uniqueness theorem for the representation of the commutation relations (10.18) that these two representation must be related by a unitary transformation. This transformation may be characterized more precisely as follows.

\section{Lemma 6.}

a) To each $\gamma \in S p(2 M, \mathbb{R})$ there exists a unitary operator $\mathrm{J}_{\gamma}$ on $L^{2}\left(\mathbb{R}^{2 M}\right)$ such that

$$
\mathrm{J}_{\gamma} \cdot \mathrm{v} \cdot \mathrm{J}_{\gamma}^{-1}=\gamma \mathrm{v}
$$

The operators $\mathrm{J}_{\gamma}$ generate a projective unitary representation of $\operatorname{Sp}(2 M, \mathbb{R})$.

b) The operators $\mathrm{J}_{\gamma}$ can be represented in the form

$$
\mathrm{J}_{\gamma}=\exp \left(i J_{\gamma}(\mathrm{v})\right)
$$

where $J_{\gamma}(\mathrm{v})$ is a quadratic expression in the operators $\mathrm{v}$.

Proof. Part a) is a classical result of I.E. Segal, [Se]. Part b) follows easily from the observation that the quadratic functions of the operators $v$ generate a representation of the Lie algebra of $S p(2 M, \mathbb{R})$ which satisfies the infinitesimal version of (10.21), see e.g. [GS] for more details.

One may therefore find an operator $\mathrm{J}_{\varphi}$ on $L^{2}\left(\mathbb{R}^{2 M}\right)$ which represents the transformation (10.19) in the sense that

$$
\mathrm{J}_{\varphi} \cdot \mathrm{v} \cdot \mathrm{J}_{\varphi}^{-1}=\hat{\mathrm{v}}(\mathrm{v})=J_{\varphi} \mathrm{v}
$$

The sought-for isomorphism $\mathrm{I}_{\varphi}$ can finally be constructed as $\mathrm{I}_{\varphi}=\mathrm{K}^{-1} \cdot \mathrm{J}_{\varphi}^{-1}$.

Remark 5. It is worth noting that the definition of $\mathcal{H}_{\mathfrak{f}}(\varphi)$ depends only on the combinatorial structure of the fat graph $\varphi$, not on the way it is embedded into the Riemann surface $\Sigma$. It follows that the isomorphism $\mathcal{H}_{\mathfrak{f}}(\mu . \varphi) \simeq \mathcal{H}_{\mathfrak{f}}(\varphi), \mu \in \mathrm{MC}(\Sigma)$ is canonical.

\section{Representations of the mapping class groups}

The representations of the mapping class group associated to the quantized Teichm uller spaces will be obtained by means of a very general construction which produces representations of the group $G$ of symmetries of a two-dimensional $\mathrm{CW}$ complex $\mathcal{G}$ out of representations of the edge path groupoid of $\mathcal{G}$. We will first describe this construction, before we discuss how to construct representations of the Ptolemy groupoid on the quantized Teichm uller spaces. The latter will then induce the sought-for representation of $\mathrm{MC}(\Sigma)$. 


\subsection{Projective unitary representations of groupoids}

Let us recall that a groupoid $\mathrm{G}$ is a category such that all morphisms are invertible. The objects of $\mathrm{G}$ will here be denoted by letters $U, V, W, \ldots$. Anticipating that the groupoids $\mathrm{G}$ we will be interested in are path groupoids of some topological space we will use the notation $[W, V] \equiv$ $\operatorname{Hom}_{\mathrm{G}}(V, W)$. The elements of $[W, V]$ will also be called "paths".

Definition 2. A unitary projective representation of the groupoid $\mathrm{G}$ consists of the following data:

(i) A Hilbert space $\mathcal{H}(V)$ associated to each object $V \in \mathrm{Ob}(\mathrm{G})$,

(ii) a map u which associates to each path $\pi \in[W, V]$ in $\mathrm{G}$ a unitary operator

$$
\mathrm{u}(\pi): \mathcal{H}(V) \rightarrow \mathcal{H}(W)
$$

(iii) a family of maps $\zeta_{V}, V \in \mathrm{Ob}(\mathrm{G})$ which associate to each closed path $\pi \in[V, V]$ a number $\zeta_{V}(\pi) \in \mathbb{C}$ with $|\zeta(\pi)|=1$.

These data are required to satisfy the relations
a) $\mathrm{u}\left(\pi_{2} \circ \pi_{1}\right)=\mathrm{u}\left(\pi_{2}\right) \cdot \mathrm{u}\left(\pi_{1}\right)$
b) $\mathrm{u}\left(\pi^{-1}\right)=\mathrm{u}^{\dagger}(\pi)$,
c) $\zeta_{V}\left(\pi_{2} \circ \pi_{1}\right)=\zeta_{V}\left(\pi_{2}\right) \zeta_{V}\left(\pi_{1}\right)$,
d) $\zeta_{V}\left(\pi^{-1}\right)=\left(\zeta_{V}(\pi)\right)^{*}$,
e) $\mathrm{u}(\pi)=\zeta_{V}(\pi)$ if $\pi \in[V, V]$,
f) $\mathrm{u}(\mathrm{id})=1$

where we use the notation $\zeta_{V}(\pi)$ also to denote the operator which multiplies each vector of $\mathcal{H}_{V}$ by the number $\zeta_{V}(\pi)$.

The groupoids of interest will be the path groupoids $\mathrm{G}$ of two-dimensional $\mathrm{CW}$ complexes $\mathcal{G}$. The set of objects is given by the set of vertices $\mathcal{G}_{\mathrm{o}}$, whereas the set of morphisms coincides with the set of paths in the complex $\mathcal{G}$. Since each path $\pi$ may be represented as a chain $E_{\pi, n(\pi)} \circ \ldots E_{\pi, 2} \circ E_{\pi, 1}$ of edges in $\mathcal{G}_{1}$ it is clear that a projective unitary representation of the path groupoid $\mathrm{G}$ of a two-dimensional $\mathrm{CW}$ complex $\mathcal{G}$ is characterized completely by specifying the images $\mathrm{u}(E)$ for $E \in \mathcal{G}_{1}$. Existence of the family of maps $\zeta_{V}$ such that relation e) is fulfilled represents a rather nontrivial constraint that the operators $\mathrm{u}(E), E \in \mathcal{G}_{1}$ have to satisfy. Of course it suffices to satisfy these constraints for the 2-cells $\pi \in \mathcal{G}_{2}$.

\subsection{Representations of symmetries of a groupoid}

The group of symmetries $G$ of a two-dimensional $\mathrm{CW}$ complex $\mathcal{G}$ is the group of all invertible mappings

$$
\mu:\left\{\begin{array}{l}
\mathcal{G}_{\mathrm{o}} \ni V \longrightarrow \mu . V \in \mathcal{G}_{\mathrm{o}}, \\
\mathcal{G}_{1} \ni E \longrightarrow \mu . E \in \mathcal{G}_{1} .
\end{array}\right.
$$

There is an associated action on the edge paths in the complex $\mathcal{G}$,

$$
[W, V] \ni \pi \rightarrow \mu . \pi \in[\mu . W, \mu . V]
$$


which is such that

$$
\mu\left(\pi_{2} \circ \pi_{1}\right)=\mu\left(\pi_{2}\right) \circ \mu\left(\pi_{1}\right), \quad \mu\left(\pi^{-1}\right)=(\mu(\pi))^{-1} .
$$

We will assume that we are given a unitary projective representation of $\mathrm{G}$ which is compatible with the symmetry $G$ in the sense that $\mathcal{H}(V)$ is canonically isomorphic with $\mathcal{H}(\mu . V), \mathcal{H}(V) \simeq$ $\mathcal{H}(\mu . V)$. We are going to show that the given representation of the groupoid $\mathrm{G}$ canonically induces a representation of its group $G$ of symmetries.

Let us fix a base point $V \in \mathrm{Ob}(\mathrm{G})$ and assume having chosen a path $\pi_{V}(\mu) \in[\mu . V, V]$ for each $\mu \in G$. Let then $\mathrm{R}(\mu): \mathcal{H}(V) \rightarrow \mathcal{H}(V) \simeq \mathcal{H}(\mu . V)$ be defined by

$$
\mathrm{R}_{V}(\mu)=\mathrm{u}\left(\pi_{V}(\mu)\right) .
$$

We are going to assume that the paths $\pi_{\mu_{1} . V}\left(\mu_{2}\right)$ are the translates of $\pi_{V}\left(\mu_{2}\right)$ under $\mu_{1}$, i.e. that $\pi_{\mu_{1} . V}\left(\mu_{2}\right)=\mu_{1} \cdot \pi_{V}\left(\mu_{2}\right)$. It follows that

$$
\mathrm{R}_{\mu_{1} . V}\left(\mu_{2}\right)=\mathrm{R}_{V}\left(\mu_{2}\right) .
$$

The operators $\mathrm{R}_{V}(\mu)$ satisfy the relations

$$
\begin{aligned}
& \mathrm{R}_{V}\left(\mu_{2}\right) \cdot \mathrm{R}_{V}\left(\mu_{1}\right)=\vartheta_{V}\left(\mu_{2}, \mu_{1}\right) \mathrm{R}_{V}\left(\mu_{2} \circ \mu_{1}\right), \\
& \vartheta_{V}\left(\mu_{2}, \mu_{1}\right)=\zeta_{V}\left(\pi_{V}^{-1}\left(\mu_{2} \circ \mu_{1}\right) \circ \pi_{\mu_{1} . V}\left(\mu_{2}\right) \circ \pi_{V}\left(\mu_{1}\right)\right) .
\end{aligned}
$$

We may next observe that the apparent dependence on the base point $V \in \mathcal{G}_{\mathrm{o}}$ is inessential. Let $V, W \in \mathcal{G}_{\mathrm{o}}$, and let us pick a path $\pi_{W, V} \in[W, V]$. For an operator $\mathrm{O}_{V}: \mathcal{H}(V) \rightarrow \mathcal{H}(V)$ we will define

$$
\mathrm{A}_{[W, V]}\left(\mathrm{O}_{V}\right)=\mathrm{u}\left(\pi_{W, V}\right) \cdot \mathrm{O}_{V}(\mu) \cdot \mathrm{u}^{\dagger}\left(\pi_{W, V}\right) .
$$

It is easy to convince oneself that $\mathrm{A}_{W, V}\left(\mathrm{O}_{V}(\mu)\right)$ does not depend on the choice of a path $\pi_{W, V} \in$ $[W, V]$. We furthermore have

$$
\mathrm{A}_{W, V}\left(\mathrm{R}_{V}(\mu)\right)=\mathrm{R}_{W}(\mu) .
$$

It easily follows that $\vartheta_{V}\left(\mu_{2}, \mu_{1}\right)$ does not depend on $V$, i.e. $\vartheta_{V}\left(\mu_{2}, \mu_{1}\right) \equiv \vartheta\left(\mu_{2}, \mu_{1}\right)$.

To summarize: The operators $\mathrm{R}_{V}(\mu)$ generate a projective unitary representation $\mathrm{R}_{V}(G)$ of $G$ on $\mathcal{H}$

$$
\mathrm{R}_{V}\left(\mu_{2}\right) \cdot \mathrm{R}_{V}\left(\mu_{1}\right)=\vartheta\left(\mu_{2}, \mu_{1}\right) \mathrm{R}_{V}\left(\mu_{2} \circ \mu_{1}\right)
$$

The operators $\mathrm{A}_{[W, V]}$ express the unitary equivalence of the representations $\mathrm{R}_{V}$ associated to the different $V \in \mathcal{G}_{\mathrm{o}}$, which allows us to regard

$$
\mathrm{R} \equiv\left[\left(\mathrm{R}_{V}\right)_{V \in \mathcal{G}_{0}},\left(\mathrm{~A}_{E}\right)_{E \in \mathcal{G}_{1}}\right]
$$

as the representation of $G$ canonically associated to the given representation of the groupoid $\mathrm{G}$.

Remark 6. There is of course some ambiguity in the construction, coming from the choice of a representative $\pi_{V}(\mu) \in[\mu . V, V]$. However, it is clearly natural to consider two representations $r, r^{\prime}$ as equivalent if the generators $r_{V}(\mu)$ and $r_{V}^{\prime}(\mu)$ differ from each other just by multiplication with a (possibly $\mu$-dependent) central element. The cocycle $\vartheta$ of the representation $r$ will differ from the cocycle $\vartheta^{\prime}$ of $r^{\prime}$ by a coboundary. 


\subsection{The projective representation of the Ptolemy groupoid on $\mathcal{K}(\varphi)$}

Following [Ka3] closely we shall define a projective representation of the Ptolemy groupoid in terms of the following set of unitary operators on $\mathcal{K}(\varphi)$

$$
\begin{aligned}
\mathrm{A}_{v} & \equiv e^{\frac{\pi i}{3}} e^{-\pi i\left(\mathbf{p}_{v}+\mathbf{q}_{v}\right)^{2}} e^{-3 \pi i \mathbf{q}_{v}^{2}} \\
\mathbf{T}_{v w} & \equiv e_{b}\left(\mathbf{q}_{v}+\mathbf{p}_{w}-\mathbf{q}_{w}\right) e^{-2 \pi i \mathbf{p}_{v} \mathbf{q}_{w}}, \quad \text { where } v, w \in \varphi_{\mathcal{o}} .
\end{aligned}
$$

The special function $e_{b}(U)$ can be defined in the strip $|\Im z|<\left|\Im c_{b}\right|, c_{b} \equiv i\left(b+b^{-1}\right) / 2$ by means of the integral representation

$$
\log e_{b}(z) \equiv \frac{1}{4} \int_{i 0-\infty}^{i 0+\infty} \frac{d w}{w} \frac{e^{-2 \mathrm{i} z w}}{\sinh (b w) \sinh \left(b^{-1} w\right)}
$$

We refer to Appendix A for more details on this remarkable special function. These operators are unitary for $(1-|b|) \Im b=0$. They satisfy the following relations [Ka3]

$$
\begin{aligned}
& \text { (i) } \quad \mathrm{T}_{v w} \mathrm{~T}_{u w} \mathrm{~T}_{u v}=\mathrm{T}_{u v} \mathrm{~T}_{v w} \text {, } \\
& \text { (ii) } \quad \mathrm{A}_{v} \mathrm{~T}_{u v} \mathrm{~A}_{u}=\mathrm{A}_{u} \mathrm{~T}_{v u} \mathrm{~A}_{v} \text {, } \\
& \text { (iii) } \mathrm{T}_{v u} \mathrm{~A}_{u} \mathrm{~T}_{u v}=\zeta \mathrm{A}_{u} \mathrm{~A}_{v} \mathrm{P}_{u v} \text {, } \\
& \text { (iv) } \mathrm{A}_{u}^{3}=\mathrm{id} \text {, }
\end{aligned}
$$

where $\zeta=e^{\pi i c_{b}^{2} / 3}, c_{b} \equiv \frac{i}{2}\left(b+b^{-1}\right)$. The relations (11.34) to (11.37) allow us to define a projective representation of the Ptolemy groupoid as follows.

- Assume that $\omega_{u v} \in\left[\varphi^{\prime}, \varphi\right]$. To $\omega_{u v}$ let us associate the operator

$$
\mathrm{u}\left(\omega_{u v}\right) \equiv \mathrm{T}_{u v}: \mathcal{K}(\varphi) \ni \mathfrak{v} \rightarrow \mathrm{T}_{u v} \mathfrak{v} \in \mathcal{K}\left(\varphi^{\prime}\right)
$$

- For each fat graph $\varphi$ and vertices $u, v \in \varphi_{\mathrm{o}}$ let us define the following operators

$$
\begin{aligned}
& \mathrm{A}_{u}^{\varphi}: \mathcal{K}(\varphi) \ni \mathfrak{v} \rightarrow \mathrm{A}_{u} \mathfrak{v} \in \mathcal{K}\left(\rho_{u} \circ \varphi\right) . \\
& \mathrm{P}_{u v}^{\varphi}: \mathcal{K}(\varphi) \ni \mathfrak{v} \rightarrow \mathrm{P}_{u v} \mathfrak{v} \in \mathcal{K}((u v) \circ \varphi) .
\end{aligned}
$$

It follows immediately from (11.34)-(11.37) that the operators $\mathrm{T}_{u v}, \mathrm{~A}_{u}$ and $\mathrm{P}_{u v}$ can be used to generate a unitary projective representation of the Ptolemy groupoid in $\mathcal{K}(\varphi) \simeq L^{2}\left(\mathbb{R}^{2 M}\right)$.

\subsection{Reduction to the quantized Teichmüller spaces}

Theorem 4. The isomorphism $\mathrm{I}_{\varphi}$ maps the operators $\mathrm{u}(\pi)$ which represent the Ptolemy groupoid on $\mathcal{K}(\varphi)$ to operators of the form $\mathrm{u}^{\prime}(\pi)=\mathrm{V}_{z}(\pi) \otimes \mathrm{V}_{h}(\pi)$, where $\mathrm{V}_{z}(\pi) \equiv\left(\mathrm{V}_{\mathfrak{f}}(\pi)\right)_{\mathfrak{f} \in B^{\prime}(\Sigma)}$ is a family of unitary operators $\mathrm{V}_{\mathfrak{f}}(\pi)$ on $\mathcal{H}_{\mathfrak{f}}(\varphi)$.

For each fixed $\mathfrak{f} \in B^{\prime}(\Sigma)$ one may use the operators $\bigvee_{\mathfrak{f}}(\pi)$ to generate a unitary projective representation of the Ptolemy groupoid. 
Proof. To begin with, let us note that each path $\pi \in \rho \equiv\left[\varphi^{\prime}, \varphi\right]$ canonically defines a map $c \mapsto c^{\prime} \equiv \mathfrak{A}(c)$ for each $c \in H_{1}(\Sigma, \mathbb{R})$. This map is defined in an obvious way for the elementary moves depicted in Figures 6, 7 if we require that $c^{\prime}$ coincides with $c$ outside the triangles depicted in these figures.

\section{Lemma 7.}

a) The map $c \mapsto \mathfrak{A}(c)$ preserves the symplectic (intersection) form on $H_{1}(\Sigma, \mathbb{R})$.

b) The operator $\mathrm{u}(\pi)$ maps $\mathrm{u}(\pi) \cdot \mathrm{h}_{\varphi, c} \cdot(\mathrm{u}(\pi))^{-1}=\mathrm{h}_{\varphi^{\prime}, \mathfrak{A}(c)}$.

Proof. Direct verifications.

Proposition 5. For each path $\pi \in\left[\varphi^{\prime}, \varphi\right]$ there exists an operator $\mathrm{H}(\pi)$ on $\mathcal{K}(\varphi)$ such that the operators $\mathrm{V}(\pi)$ on $L^{2}\left(\mathbb{R}^{2 M}\right)$ defined by

$$
\mathrm{V}(\pi)=\mathrm{H}(\pi) \cdot \mathrm{U}(\pi), \quad \mathrm{U}(\pi) \equiv \mathrm{J}_{\varphi^{\prime}}^{-1} \cdot \mathrm{u}(\pi) \cdot \mathrm{J}_{\varphi},
$$

(i) commute with all operators $\mathrm{h}_{m}, \mathrm{~h}_{m}^{\vee}, m=1, \ldots, g$ and $\mathrm{f}_{l}, l=1, \ldots, s-1$,

(ii) generate a unitary projective representation of $\operatorname{Pt}(\Sigma)$ on $L^{2}\left(\mathbb{R}^{2 M}\right)$.

The operators $\mathrm{H}(\pi)$ can be represented in the form

$$
\mathbf{H}(\pi) \equiv \exp \left(i H_{\pi}\left(\mathbf{h}_{\varphi^{\prime}}\right)\right)
$$

where $H_{\pi}\left(\mathrm{h}_{\varphi^{\prime}}\right)$ is a quadratic function of the $2 g$ operators $\mathrm{h}_{1}^{\prime}, \ldots, \mathrm{h}_{g}^{\prime}, \mathrm{h}_{1}^{\prime \vee}, \ldots, \mathrm{h}_{g}^{\prime \vee}$ on $\mathcal{K}\left(\varphi^{\prime}\right)$.

Proof. The existence of operators $\mathrm{H}(\pi)$ of the form (11.38) which are such that statement (i) of the Proposition is verified follows directly from Lemma 6 if one takes into account that the transformation $\mathrm{h}_{\varphi^{\prime}, c} \rightarrow \mathrm{h}_{\varphi^{\prime}, c^{\prime}}$ is represented by an element of $S p(g, \mathbb{R})$ according to part a) of Lemma 7.

In order to prove statement (ii) of the proposition, we mainly need to check that the operators $\mathrm{V}(\pi)$ satisfy the relations of the Ptolemy groupoid. Let us consider a closed path $\pi \in[\varphi, \varphi]$ which decomposes into a chain of edges as $\pi=\pi_{n} \circ \cdots \circ \pi_{1}$.

$$
\mathrm{V}(\pi) \equiv \mathrm{V}\left(\pi_{n}\right) \cdots \mathrm{V}\left(\pi_{1}\right)
$$

On the one hand one may observe that $\mathrm{V}(\pi)$ can be factorized as

$$
\mathrm{V}(\pi)=\mathrm{H}(\pi) \cdot \mathrm{U}\left(\pi_{n}\right) \cdots \mathrm{U}\left(\pi_{1}\right)=\mathrm{H}(\pi) \zeta_{\varphi}(\pi)
$$

The operator $\mathrm{H}(\pi)$ in (11.39) can be represented as follows:

$$
\mathrm{H}(\pi)=\mathrm{H}\left(\pi_{n}\right) \cdot\left[\mathrm{U}\left(\pi_{n}^{\prime}\right) \cdot \mathrm{H}\left(\pi_{n-1}\right) \cdot \mathrm{U}\left(\pi_{n}^{\prime}\right)^{\dagger}\right] \cdots\left[\mathrm{U}\left(\pi_{1}^{\prime}\right) \cdot \mathrm{H}\left(\pi_{1}\right) \cdot \mathrm{U}\left(\pi_{1}^{\prime}\right)^{\dagger}\right],
$$

where $\pi_{n-k}^{\prime} \equiv \pi_{n} \circ \cdots \circ \pi_{n-k}$. It follows from Lemma 7 together with (11.38) that

$$
\mathrm{U}\left(\pi_{j+1}^{\prime}\right) \cdot \mathrm{H}\left(\pi_{j}\right) \cdot \mathrm{U}\left(\pi_{j+1}^{\prime}\right)^{\dagger}=\exp \left(i H_{\pi_{j}}\left(\mathrm{~h}_{\varphi}\right)\right)
$$


Taking into account equation (10.16) we conclude that

$$
\mathrm{K}^{-1} \cdot \mathrm{H}(\pi) \cdot \mathrm{K}=1 \otimes \mathrm{H}_{h}(\pi) .
$$

On the other hand let us note that $\mathrm{V}(\pi)$ commutes with all operators $\mathrm{h}_{m}, \mathbf{h}_{m}^{\vee}, m=1, \ldots, g$. Equation (11.39) implies that the same is true for $\mathrm{H}(\pi)$. However, the representation of the operators $\mathrm{h}_{m}, \mathrm{~h}_{m}^{\vee}, m=1, \ldots, g$ on $\mathcal{H}_{h}(\varphi)$ is irreducible (see Remark 3). This allows us to conclude that $\mathrm{H}(\pi)=\eta_{\varphi}(\pi) \in \mathbb{C},\left|\eta_{\varphi}(\pi)\right|=1$. Inserting this into (11.39) proves our claim.

It follows from statement (i) in Proposition 5 that

$$
\mathrm{K}^{-1} \cdot \mathrm{V}(\pi) \cdot \mathrm{K}=\mathrm{V}_{z}(\pi) \otimes 1
$$

The task remains to describe the operators $\mathrm{V}_{z}(\pi)$ more precisely.

Proposition 6. There exists a family of unitary operators $\bigvee_{\mathfrak{f}}(\pi): \mathcal{H}_{\mathfrak{f}}(\varphi) \rightarrow \mathcal{H}_{\mathfrak{f}}\left(\varphi^{\prime}\right), \mathfrak{f} \in B^{\prime}(\Sigma)$ which represent the action of the operator $\mathrm{V}_{z}(\pi): \mathcal{H}_{z}(\varphi) \rightarrow \mathcal{H}_{z}\left(\varphi^{\prime}\right)$.

Proof. To begin with, let us observe that it follows from (11.32) that the operators $\mathrm{u}(E)$ associated to the edges $E=\rho_{v}, E=\omega_{v w}$ in $\operatorname{Pt}_{1}(\Sigma)$ can all be factorized as $\mathrm{u}(E)=\mathrm{Q}(E) \cdot G\left(\hat{\mathrm{z}}_{e}\right)$, where $\mathrm{Q}(E)$ is of the form $\mathrm{Q}(E)=\exp \left(i Q_{E}(\mathrm{v})\right)$ for a quadratic function $Q_{E}$, and $G \equiv 1$ if $E=\rho_{v}$ and $G(z)=e_{b}(z)$ if $E=\omega_{v w}$. It follows that the corresponding operator $\mathrm{V}(E)$ defined in Proposition 5 can be factorized as

$$
\mathrm{V}(E)=\mathrm{Q}^{\prime}(E) \cdot G\left(\mathrm{z}_{e}\right) \text {, where } \mathrm{Q}^{\prime}(E) \equiv \mathrm{H}(E) \cdot \mathrm{J}_{\varphi^{\prime}}^{-1} \cdot \mathrm{Q}(E) \cdot \mathrm{J}_{\varphi} .
$$

The operator $\mathrm{Q}^{\prime}(E)$ is a product of four operators $\mathrm{J}_{\gamma_{k}}, \gamma_{k} \in S p(2 M, \mathbb{R})$ for $k=1,2,3,4$. If follows from Lemma 6 that it can be represented in the form

$$
\mathrm{Q}^{\prime}(E)=\exp \left(i J_{E}(\mathrm{v})\right)
$$

for some expression $J_{E}(\mathrm{v})$ which is quadratic in v. Note that the operators $G\left(\mathrm{z}_{e}\right)$ and $\mathrm{V}(E)$ commute with all operators $\mathrm{h}_{m}, \mathrm{~h}_{m}^{\vee}, m=1, \ldots, g$ and $\mathrm{f}_{l}, l=1, \ldots, s-1$. It follows that the same is true for $\mathrm{Q}^{\prime}(E)$, which implies that $J_{E}(\mathrm{v}) \equiv J_{E}(\mathrm{z})$ depends only on the vector $\mathbf{z} \equiv\left(\mathbf{z}_{e}\right)_{e \in \varphi_{1}} \cdot \mathrm{V}(E)$ is therefore of the form $\mathrm{V}(E)=\exp \left(i J_{E}(\mathbf{z})\right) \cdot G\left(\mathbf{z}_{e}\right)$. Our claim follows easily, $\left.\mathbf{W}_{\mathfrak{f}}(E)=\exp \left(i J_{E}\left(\mathbf{z}_{\mathfrak{f}, e}\right)\right) \cdot G\left(\mathbf{z}_{\mathfrak{f}, e}\right)\right)$ does the job.

Theorem 4 follows by combining Propositions 5 and 6.

We are finally in the position to define more precisely what we will regard as the quantized Teichm üller spaces. To this aim let us note that the Hilbert spaces $\mathcal{H}_{0}(\varphi)$ associated to the origin o in $B^{\prime}(\Sigma)$ form irreducible representations of the relations (10.6), (10.7). Funktions of the operators $\mathrm{z}_{\mathrm{o}, e}$ generate the algebras $\mathbf{B}\left(\mathcal{H}_{\mathrm{o}}(\varphi)\right)$ of all bounded operators on $\mathcal{H}_{\mathrm{o}}(\varphi)$, which suggests to interprete $\mathbf{B}\left(\mathcal{H}_{\mathcal{o}}(\varphi)\right)$ as particular representations of the quantized algebras $\mathcal{T}_{\hbar}(\Sigma)$ of functions on the Teichm uller spaces.

The operators $\mathrm{V}_{\mathrm{o}}(\pi)$ generate a unitary projective representation of the Ptolemy groupoid which allows us to regard two operators $\mathrm{O}_{\varphi_{2}} \in \mathbf{B}\left(\mathcal{H}_{\mathrm{o}}\left(\varphi_{2}\right)\right)$ and $\mathrm{O}_{\varphi_{1}} \in \mathbf{B}\left(\mathcal{H}_{\mathrm{o}}\left(\varphi_{1}\right)\right)$ as equivalent, $\mathrm{O}_{\varphi_{2}} \sim \mathrm{O}_{\varphi_{1}}$, iff

$$
\mathrm{O}_{2}=\mathrm{V}_{\mathrm{o}}(\pi) \cdot \mathrm{O}_{1} \cdot\left(\mathrm{V}_{\mathrm{o}}(\pi)\right)^{-1}, \quad \pi \in\left[\varphi_{2}, \varphi_{1}\right]
$$


Let furthermore $\mathrm{MC}_{\varphi}$ be the unitary projective representation of the mapping class group $\mathrm{MC}(\Sigma)$ which is generated from the operators $V_{\mathfrak{o}}(\pi)$ by means of the construction in Subsection 11.2 .

\section{Definition 3.}

(i) We define the algebra $\mathcal{T}_{\hbar}(\Sigma)$ as the algebra generated by the families $\mathcal{O} \equiv\left(\mathrm{O}_{\varphi}\right)_{\varphi \in \mathcal{P} t_{\mathcal{o}}(\Sigma)}$ of bounded operators $\mathrm{O}_{\varphi}$ on $\mathcal{H}_{\mathrm{o}}(\varphi)$ such that $\mathrm{O}_{\varphi_{2}} \sim \mathrm{O}_{\varphi_{1}}$ for all $\varphi_{2}, \varphi_{1} \in \mathcal{P} t_{\mathrm{o}}(\Sigma)$. The algebra $\mathcal{T}_{\hbar}(\Sigma)$ will be called the quantized algebra of functions on the Teichmüller spaces.

(ii) Let $\mathcal{M C}_{\hbar}(\Sigma)$ be the subalgebra of $\mathcal{T}_{\hbar}(\Sigma)$ generated by the families $\mathcal{M C}(\mu) \equiv$ $\left(\operatorname{MC}_{\varphi}(\mu)\right)_{\varphi \in \mathcal{P} t_{\mathrm{o}}(\Sigma)}$ for all $\mu \in \mathrm{MC}(\Sigma)$. 


\section{Part IV}

\section{A stable modular functor from the quantum Teichmüller spaces}

Let us recall that systems of Fenchel-Nielsen coordinates are naturally associated to markings of the surfaces $\Sigma$. The transformations between the different markings of a Riemann surface $\Sigma$ generate yet another groupoid, called the stable modular groupoid. Given that the quantum version of the changes between the Penner coordinates associated to different fat graphs was represented by a unitary projective representation of the Ptolemy groupoid it is natural to expect that the quantization of the Fenchel-Nielsen coordinates should similarly come with a unitary projective representation of the modular groupoid.

Pants decompositions have one big advantage over ideal triangulations: The gluing operation allows us to build "larger" surfaces from simple pieces of the same type, namely hyperbolic surfaces with holes. It is natural to require that the unitary projective representations of the modular groupoid assigned to surfaces $\Sigma$ should correspondingly be organized in a "towerlike" fashion: They should allow restriction to, and should be generated by the representations assigned to the surfaces $\Sigma^{\prime}$ which are obtained from $\Sigma$ by cutting along simple closed curves on $\Sigma$.

Our aim in the rest of this paper will be to show that such a structure can be constructed from the quantized Teichm uller spaces considered in the previous part of this paper. This is of great importance since

\section{having a tower of projective unitary representations of the stable modular groupoid is equivalent to having a stable unitary modular functor.}

The notion of a stable unitary modular functor will be introduced in the next section. One main difference to the more conventional (two-dimensional) modular functors as defined e.g. in [Tu, BK2] is that one restricts attention to Riemann surfaces $\Sigma$ of genus $g$ and with $n$ parametrized boundary components which are stable in the sense that $2 g-2+n>0$.

We will then explain why having a stable unitary modular functor is equivalent to having a tower of projective unitary representations of the modular groupoid before we take up the task to actually construct the latter from the quantization of the Teichm uller spaces as described previously.

\section{The notion of a stable unitary modular functor}

Given that the usual definitions of a modular functor take several pages to fully write them down [Tu, BK2], we shall only briefly explain the most important features. The missing details will not differ much from the definitions discussed in [Tu, BK2]. 


\subsection{Rigged Riemann surfaces}

We will consider compact oriented surfaces $\Sigma$ with boundary $\partial \Sigma=\coprod_{\beta \in A(\Sigma)} b_{\beta}$, where $A(\Sigma) \equiv$ $\pi_{0}(\partial \Sigma)$ is the set of connected components of $\partial \Sigma$. A surface $\Sigma$ is called an extended surface if one has chosen orientation-preserving homeomorphisms $p_{\beta}: b_{\beta} \rightarrow S^{1}$ for each connected component $b_{\beta}$ of the boundary. To be concrete, let $S^{1}=\{z \in \mathbb{C} ;|z|=1\}$. An e-surface $\Sigma$ of genus $g$ and with $n$ boundary circles is called stable if $2 g-2+n>0$.

We will use the terminology rigged Riemann surface, or r-surface for short, for triples $\widehat{\Sigma}=$ $(\Sigma, y, \mathfrak{c})$, where

$\triangleright \Sigma$ is a stable extended surface,

$\triangleright y$ is a Lagrangian subspace of $H_{1}(\Sigma, \mathbb{R})$, and

$\triangleright \mathfrak{c}: A(\Sigma) \rightarrow \mathcal{L}$ is a coloring of the boundary of $\Sigma$ by elements of a set $\mathcal{L}$.

Given an r-surface $\widehat{\Sigma}$ and given $\beta, \beta^{\prime} \in A(\Sigma)$ such that $\mathfrak{c}(\beta)=\mathfrak{c}\left(\beta^{\prime}\right)$ we can define a new rsurface $\widehat{\Sigma}^{\prime} \equiv \sqcup_{\beta \beta^{\prime}} \widehat{\Sigma}=\left(\Sigma^{\prime}, y^{\prime}, \mathfrak{c}^{\prime}\right)$ which is obtained from $\widehat{\Sigma}$ by gluing the boundary components $b_{\beta}$ and $b_{\beta^{\prime}}$.

$\triangleright$ The surface $\Sigma^{\prime} \equiv \sqcup_{\beta \beta^{\prime}} \Sigma$ is defined by identifying all points $p \in b_{\beta}$ with $\left(p_{\beta^{\prime}}^{-1} \circ p_{\beta}^{o}\right)(p) \in b_{\beta^{\prime}}$, where $p_{\beta}^{o}: b_{\beta} \rightarrow S^{1}$ is defined by $p_{\beta}^{o}(p)=-\overline{p_{\beta}(p)}$. There is a corresponding projection $P_{\beta \beta^{\prime}}: \Sigma \rightarrow \Sigma^{\prime} \equiv \sqcup_{\beta \beta^{\prime}} \Sigma$ which maps $b_{\beta}, b_{\beta^{\prime}}$ to the same simple closed curve on $\Sigma^{\prime}$.

$\triangleright$ The Lagrangian subspace $y^{\prime}$ is given by the image of $H_{1}(\Sigma, \mathbb{R})$ under the projection $P_{\beta \beta^{\prime}}$,

$\triangleright$ The coloring $\mathfrak{c}^{\prime}$ is obtained from $\mathfrak{c}$ by putting $\mathfrak{c}^{\prime}\left(\alpha^{\prime}\right)=\mathfrak{c}(\alpha)$ if $P_{\beta \beta^{\prime}}\left(b_{\alpha}\right)=b_{\alpha^{\prime}} \in \partial \Sigma^{\prime}$.

\subsection{Stable unitary modular functors}

Let $\mathcal{L}$ now be a space with a measure $d \nu$. A stable modular functor with central charge is the following collection of data.

$\triangleright$ Assignment $\widehat{\Sigma}=(\Sigma, y, \mathfrak{c}) \rightarrow \mathcal{H}(\Sigma, y, \mathfrak{c})$, where

- $\widehat{\Sigma}$ is an r-surface,

- $\mathcal{H}(\Sigma, y, \mathfrak{c})$ is a separable Hilbert space,

$\triangleright$ Mapping class group: Assignment

$$
[f] \longrightarrow\left(\mathrm{U}_{[f]}: \mathcal{H}(\Sigma, y, \mathfrak{c}) \rightarrow \mathcal{H}\left(\Sigma^{\prime}, y_{f}^{\prime}, \mathfrak{c}_{f}^{\prime}\right)\right)
$$

- $[f]$ is the isotopy class of a homeomorphism $f: \Sigma \rightarrow \Sigma^{\prime}$,

- $\mathrm{U}_{[f]}$ is a unitary operator,

- $y_{f}^{\prime}$ is the Lagrangian subspace of $H_{1}\left(\Sigma^{\prime}, \mathbb{R}\right)$ determined from $y$ via $f$,

$-\mathfrak{c}_{f}^{\prime}: A\left(\Sigma^{\prime}\right) \rightarrow \mathcal{L}:$ the coloring of boundary components of $\Sigma^{\prime}$ induced from $\mathfrak{c}$ via $f$. 
$\triangleright$ Disjoint union: There exist unitary operators

$$
\mathrm{G}_{21}: \mathcal{H}\left(\Sigma_{2} \sqcup \Sigma_{1}, y_{2} \oplus y_{1}, \mathfrak{c}_{2} \sqcup \mathfrak{c}_{1}\right) \stackrel{\sim}{\longrightarrow} \mathcal{H}\left(\Sigma_{2}, y_{2}, \mathfrak{c}_{2}\right) \otimes \mathcal{H}\left(\Sigma_{1}, y_{1}, \mathfrak{c}_{1}\right) .
$$

$\triangleright$ Gluing: Let $\left(\Sigma^{\prime}, y^{\prime}\right)$ be obtained from $(\Sigma, y)$ by gluing of two boundary components $\alpha, \beta$. There then exists a unitary operator

$$
\mathrm{G}_{\alpha \beta}: \int_{\mathcal{L}} d \nu(s) \mathcal{H}\left(\Sigma, y, \mathfrak{c}_{s\lfloor\alpha \beta}\right) \stackrel{\sim}{\longrightarrow} \mathcal{H}\left(\Sigma^{\prime}, y^{\prime}, \mathfrak{c}^{\prime}\right)
$$

where the coloring $\mathfrak{c}_{s\lfloor\alpha \beta}: A(\Sigma) \rightarrow \mathcal{L}$ is defined from $\mathfrak{c}^{\prime}$ via

$$
\begin{aligned}
& \mathfrak{c}_{s\lfloor\alpha \beta}(c)=\mathfrak{c}^{\prime}\left(P_{\alpha \beta}(c)\right) \quad \text { if } \quad c \in A(\Sigma) \backslash\{\alpha, \beta\}, \\
& \mathfrak{c}_{s\lfloor\alpha \beta}(c)=s \quad \text { if } \quad c \in\{\alpha, \beta\} .
\end{aligned}
$$

These data are required to satisfy the following "obvious" consistency and compatibility conditions:

Multiplicativity: For all homeomorphisms $f: \Sigma_{1} \rightarrow \Sigma_{2}, g: \Sigma_{2} \rightarrow \Sigma_{3}$ there exists $\zeta(f, g) \in S^{1}$ such thay we have

$$
\mathrm{U}_{[f]} \mathrm{U}_{[g]}=\zeta(f, g) \mathbf{U}_{[f \circ g]} .
$$

$\zeta(f, g)$ has to satisfy the condition $\zeta(f, g) \zeta(f \circ g, h)=\zeta(g, h) \zeta(f, g \circ h)$.

Functoriality: The gluing isomorphisms and the disjoint union isomorphisms are functorial in $\Sigma .{ }^{7}$

Compatibility: The gluing isomorphisms and the disjoint union isomorphisms are mutually compatible.

Symmetry of Gluing: $G_{\alpha \beta}=G_{\beta \alpha}$.

It would take us several pages to write out all conditions in full detail, we therefore refer to [Tu, BK2] for more details. However, it seems that the following two "naturality" requirements represent a key to the understanding of the notion of the modular functor:

\section{Naturality:}

a) Let $f_{1}: \Sigma_{1} \rightarrow \Sigma_{1}^{\prime}, f_{2}: \Sigma_{2} \rightarrow \Sigma_{2}^{\prime}$ be r-homeomorphisms. We then have

$$
\mathrm{G}_{21} \cdot \mathrm{U}_{\left[f_{2} \sqcup f_{1}\right]} \cdot \mathrm{G}_{21}^{\dagger} \equiv \mathrm{U}_{\left[f_{2}\right]} \otimes \mathrm{U}_{\left[f_{1}\right]} \cdot
$$

b) If the r-homeomorphism $f: \Sigma_{1} \rightarrow \Sigma_{2}$ induces an r-homeomorphism $f^{\prime}: \Sigma_{1}^{\prime} \rightarrow \Sigma_{2}^{\prime}$ of the surfaces $\Sigma_{1}^{\prime}, \Sigma_{2}^{\prime}$ obtained from $\Sigma_{1}, \Sigma_{2}$ by the gluing construction, we have

$$
\mathrm{G}_{\alpha \beta} \cdot\left(\int_{\mathcal{L}} d \nu(s) \mathrm{U}_{[f]}\left(\Sigma, y, \mathfrak{c}_{s\lfloor\alpha \beta}\right)\right) \cdot \mathrm{G}_{\alpha \beta}^{\dagger}=\mathrm{U}_{\left[f^{\prime}\right]}\left(\Sigma^{\prime}, y^{\prime}, \mathfrak{c}^{\prime}\right)
$$

\footnotetext{
${ }^{7}$ One is considering the category with objects r-surfaces, and morphisms isotopy classes of homeomorphisms of r-surfaces, equipped additionally with the gluing and disjoint union operations.
} 
These requirements make clear how the mapping class group representations on the spaces $\mathcal{H}\left(\Sigma^{\prime}, y^{\prime}, \mathfrak{c}^{\prime}\right)$ restrict to and are generated by the representations assigned to the surfaces $\Sigma$ which are obtained from $\Sigma^{\prime}$ by cutting along simple closed curves on $\Sigma^{\prime}$.

Remark 7. The standard definitions of modular functors assume that the Hilbert spaces $\mathcal{H}(\Sigma, y, \mathfrak{c})$ are finite-dimensional. They are therefore not suitable for nonrational conformal field theories. Our definition should be seen as a first step towards the definition of analogs of modular functors which are associated to nonrational conformal field theories in a way similar to the connections between rational conformal field theories and modular functors mentioned in the introduction.

However, there is one important ingredient of the usual definition that does not have an obvious counterpart in our framework. In the more standard definitions of modular functors it is required that there exists a distinguished element $s_{\mathrm{o}}$ in $\mathcal{L}$ which has the property that coloring a boundary component $\beta$ with $s_{\mathrm{o}}$ is equivalent to "closing" this boundary component. More precisely, let $\mathfrak{c}_{s\lfloor\beta}$ be a coloring of the boundary components of an extended surface $\Sigma$ which assigns $s \in \mathcal{L}$ to the component with label $\beta$, and let $\widehat{\Sigma}_{\breve{\beta}}=\left(\Sigma_{\breve{\beta}}, y_{\breve{\beta}}, \mathfrak{c}_{\breve{\beta}}\right)$ be the r-surface obtained from $\widehat{\Sigma}=(\Sigma, y, \mathfrak{c})$ by gluing a disc to $b_{\beta}$. The more standard definitions of modular functors assume or imply existence of an element $s_{\mathrm{o}}$ in $\mathcal{L}$ such that

$$
\mathcal{H}\left(\Sigma, y, \mathfrak{c}_{s_{\mathrm{o}}\lfloor\beta}\right) \simeq \mathcal{H}\left(\Sigma_{\breve{\beta}}, y_{\breve{\beta}}, \mathfrak{c}_{\breve{\beta}}\right), \quad \mathrm{U}_{[f]}\left(\Sigma, y, \mathfrak{c}_{s_{\diamond}\lfloor\beta}\right) \simeq \mathrm{U}_{[f]}\left(\Sigma_{\breve{\beta}}, y_{\breve{\beta}}, \mathfrak{c}_{\breve{\beta}}\right)
$$

This yields additional relations between the mapping class group representations assigned to surfaces with different numbers of boundary components.

In the case of the quantized Teichm uller spaces it ultimately turns out that an analog of the element $s_{\mathfrak{o}}$ in $\mathcal{L}$ exists only if one considers the analytic continuation of $\mathcal{H}(\Sigma, y, \mathfrak{c})$ and $\mathrm{U}_{[f]}(\Sigma, y, \mathfrak{c})$ with respect to the boundary labels $\mathfrak{c}(\beta) \in \mathcal{L}$. This fact, and the corresponding improvement of our definition of a stable modular functor will be elaborated upon elsewhere.

\subsection{Representations of the modular groupoid versus modular functors}

It turns out to be very useful to reformulate the notion of a stable unitary modular functor in terms of generators and relations as follows. Any surface $\Sigma^{\prime}$ can be glued from a surface $\Sigma_{\mathcal{o}}=\coprod_{p \in \sigma_{\mathrm{o}}} T_{p}$ which is a disjoint union of trinions. The different ways of doing this can be parametrized ${ }^{8}$ by markings $\sigma$. The gluing construction determines a canonical Lagrangian subspace $y_{\sigma}^{\prime}$ of $H_{1}\left(\Sigma^{\prime}, \mathbb{R}\right)$ from the tautological Lagrangian subspace $y_{\mathfrak{o}} \equiv H_{1}\left(\Sigma_{\mathfrak{o}}, \mathbb{R}\right)$. By iterating the gluing and disjoint union isomorphisms one defines unitary operators $\mathrm{G}\left(\sigma, \mathfrak{c}^{\prime}\right)$ : $\mathcal{H}\left(\Sigma^{\prime}, y_{\sigma}^{\prime}, \mathfrak{c}^{\prime}\right) \rightarrow \mathcal{H}\left(\sigma, \mathfrak{c}^{\prime}\right)$, where

$$
\mathcal{H}\left(\sigma, \mathfrak{c}^{\prime}\right) \equiv \int_{\mathfrak{L}} d \nu_{\sigma}(\mathrm{S}) \bigotimes_{p \in \sigma_{\mathfrak{o}}} \mathcal{H}\left(T_{p}, \mathfrak{c}_{\sigma, p}^{\mathrm{S}}\right)
$$

We have used the following notation:

\footnotetext{
${ }^{8}$ Note that markings with the same cut system will yield equivalent representations for $\Sigma^{\prime}$. This redundancy will give a useful book-keeping device when the representation of the mapping class group is considered.
} 
- The integration is extended over the set $\mathfrak{L}$ of all colorings $\mathrm{S}: \mathcal{C}_{\sigma} \ni c \rightarrow s_{c} \in \mathcal{L}$ equipped with the canonical product measure $d \nu_{\sigma}(\mathrm{S})$ obtained from $d \nu$ by choosing any numbering of the elements of $\mathcal{C}_{\sigma}$.

- $\mathfrak{c}_{\sigma, p}^{\mathrm{S}}$ is the coloring of the boundary components of $T_{p}$ which is defined by assigning

$$
\mathfrak{c}_{\sigma, p}^{\mathrm{S}}(\beta)=\left\{\begin{array}{l}
\mathfrak{c}^{\prime}\left(\beta^{\prime}\right) \text { if } P_{\sigma, p}(\beta)=\beta^{\prime} \in A\left(\Sigma^{\prime}\right), \\
s_{c} \text { if } P_{\sigma, p}(\beta)=c \in \mathcal{C}_{\sigma} .
\end{array}\right.
$$

$P_{\sigma, p}(\beta)$ is the embedding $T_{p} \hookrightarrow \Sigma^{\prime}$ defined from $\sigma$ by the gluing construction.

Unitary mappings between the different spaces $\mathcal{H}(\sigma, \mathfrak{c})$ arise in two ways: First, one may have different markings $\sigma_{2}, \sigma_{1}$ such that the Lagrangian subspaces defined by the gluing construction coincide, $y_{\sigma_{1}}=y_{\sigma_{2}}$. In this case one gets unitary operators $F_{\sigma_{2} \sigma_{1}}(\mathfrak{c}): \mathcal{H}\left(\sigma_{1}, \mathfrak{c}\right) \rightarrow \mathcal{H}\left(\sigma_{2}, \mathfrak{c}\right)$ from the composition

$$
\mathrm{F}_{\sigma_{2} \sigma_{1}}(\mathfrak{c}) \equiv \mathrm{G}\left(\sigma_{2}, \mathfrak{c}\right) \cdot\left(\mathrm{G}\left(\sigma_{1}, \mathfrak{c}\right)\right)^{\dagger}
$$

Secondly, one has the mappings $\bigcup_{[f]}$ which may map between spaces $\mathcal{H}\left(\Sigma, y_{\sigma}, \mathfrak{c}\right)$ and $\mathcal{H}\left(\Sigma^{\prime},\left(y_{\sigma}\right)_{f}^{\prime}, \mathfrak{c}_{f}^{\prime}\right)$. In the case that $\mu: \Sigma \rightarrow \Sigma$ represents an element of the mapping class group it is natural to define operators $\mathrm{V}_{\sigma \mu}(\mathfrak{c}): \mathcal{H}(\sigma, \mathfrak{c}) \rightarrow \mathcal{H}\left(\sigma_{\mu}^{\prime}, \mathfrak{c}_{\mu}^{\prime}\right)$ by

$$
\mathrm{V}_{\sigma \mu}(\mathfrak{c})=\mathrm{G}\left(\sigma_{\mu}^{\prime}, \mathfrak{c}_{\mu}^{\prime}\right) \cdot \mathrm{U}_{[\mu]} \cdot(\mathrm{G}(\sigma, \mathfrak{c}))^{\dagger},
$$

where $\sigma_{\mu}^{\prime}$ is the image of the marking $\sigma$ under $\mu$.

It turns out - as will be reviewed in the next section - that there exists a set $\mathcal{M}_{1}(\Sigma)$ of elementary moves between the different markings $\sigma$ such that any two markings $\sigma_{2}, \sigma_{1}$ can be connected by paths $\pi$ which are composed out of the elementary moves. There furthermore exists a set $\mathcal{M}_{2}(\Sigma)$ of relations which makes the resulting two-dimensional $\mathrm{CW}$ complex $\mathcal{M}(\Sigma)$ simply connected. The correponding path groupoid is called the modular groupoid and denoted by $\mathrm{M}(\Sigma)$.

Our construction of the operators $\mathrm{F}_{\sigma_{2} \sigma_{1}}(\mathfrak{c}), \mathrm{V}_{\sigma \mu}(\mathfrak{c})$ assigns unitary operators to each of the elementary moves. This yields unitary operators $\mathrm{U}(\pi, \mathfrak{c}): \mathcal{H}\left(\sigma_{1}, \mathfrak{c}\right) \rightarrow \mathcal{H}\left(\sigma_{2}, \mathfrak{c}_{\pi}\right)$ for each path $\pi \in\left[\sigma_{2}, \sigma_{1}\right]$, where $\mathfrak{c}_{\pi}$ is the coloring of boundary components which is obtained from $\mathfrak{c}$ by tracking the relabeling of boundary components defined by $\pi$. Due to the fact that multiplicativity holds only projectively, cf. eqn. (12.1), one will find that there exists $\zeta(\pi) \in S^{1}$ such that $\mathrm{U}(\pi)=\zeta(\pi)$ for all closed paths, starting and ending at the same marking $\sigma$. In other words, a stable unitary modular functor canonically defines projective unitary representations of the stable modular groupoid.

Conversely, the definition of a tower of projective unitary representations of the modular groupoids $\mathrm{M}(\Sigma)$ involves the following data.

$\triangleright$ Assignment $(\sigma, \mathfrak{c}) \rightarrow \mathcal{H}(\sigma, \mathfrak{c})$, where $\mathcal{H}(\sigma, \mathfrak{c})$ is constructed out of spaces $\mathcal{H}\left(S_{3}, \mathfrak{c}_{3}\right)$ assigned to trinions as in (12.2).

$\triangleright$ Assignment

$$
\pi \in\left[\sigma_{2}, \sigma_{1}\right] \longrightarrow\left(\mathrm{U}(\pi, \mathfrak{c}): \mathcal{H}\left(\sigma_{1}, \mathfrak{c}\right) \rightarrow \mathcal{H}\left(\sigma_{2}, \mathfrak{c}_{\pi}\right)\right)
$$


It is required that the operators $\mathrm{U}(\pi, \mathfrak{c})$ generate unitary projective representations of the modular groupoids associated to the surfaces $\Sigma$. In order to formulate the additional requirements which turn this collection of representations of $\mathrm{M}(\Sigma)$ into a tower let us note that markings can be glued in a natural way. We will use the notation $\sqcup_{\alpha \beta} \sigma$ for the marking obtained by gluing boundary components $\alpha$ and $\beta$. Gluing and disjoint union now become realized in a trivial manner,

$$
\begin{aligned}
& \mathcal{H}\left(\sigma_{2} \sqcup \sigma_{1}, \mathfrak{c}_{2} \sqcup \mathfrak{c}_{1}\right)=\mathcal{H}\left(\sigma_{2}, \mathfrak{c}_{2}\right) \otimes \mathcal{H}\left(\sigma_{1}, \mathfrak{c}_{1}\right), \\
& \mathcal{H}\left(\sqcup_{\alpha \beta} \sigma, \mathfrak{c}^{\prime}\right)=\int_{\mathcal{L}} d \nu(s) \mathcal{H}\left(\sigma, \mathfrak{c}_{s\lfloor\alpha \beta}\right) .
\end{aligned}
$$

The data specified above are then required to satisfy the following naturality conditions.

\section{Naturality:}

a) Let $\pi=\pi_{2} \sqcup \pi_{1} \in\left[\sigma_{2}^{\prime}, \sigma_{2}\right] \sqcup\left[\sigma_{1}^{\prime}, \sigma_{1}\right]$ We then have

$$
\mathrm{U}\left(\pi_{2} \sqcup \pi_{1}, \mathfrak{c}_{2} \sqcup \mathfrak{c}_{1}\right) \equiv \mathrm{U}\left(\pi_{2}, \mathfrak{c}_{2}\right) \otimes \mathrm{U}\left(\pi_{1}, \mathfrak{c}_{1}\right) .
$$

b) Given a path $\pi \in\left[\sigma_{2}, \sigma_{1}\right]$ in $\mathcal{M}(\Sigma)$ let $\sqcup_{\alpha \beta} \pi \in\left[\sqcup_{\alpha \beta} \sigma_{2}, \sqcup_{\alpha \beta} \sigma_{1}\right]$ be the corresponding path in $\mathcal{M}\left(\sqcup_{\alpha \beta} \Sigma\right)$ defined by the gluing construction. We then have

$$
\mathrm{U}\left(\sqcup_{\alpha \beta} \pi, \mathfrak{c}^{\prime}\right)=\int_{\mathcal{L}} d \nu(s) \mathrm{U}\left(\pi, \mathfrak{c}_{s\lfloor\alpha \beta}\right)
$$

From a tower of projective unitary representations of the modular groupoids one can reconstruct a stable unitary modular functor as follows. The system of isomorphisms $\mathrm{U}(\pi, \mathfrak{c})$ : $\mathcal{H}\left(\sigma_{1}, \mathfrak{c}\right) \rightarrow \mathcal{H}\left(\sigma_{2}, \mathfrak{c}\right)$ allows us to identify the Hilbert spaces associated to different markings $\sigma$, thereby defining $\mathcal{H}(\Sigma, \mathfrak{c})$. The representation of the modular groupoid defines a representation of the mapping class group on $\mathcal{H}(\Sigma, \mathfrak{c})$ via the construction in subsection 11.1. We refer to [BK2] for more details.

It is important to note (see next section for a detailed discussion) that the elements of the set $\mathcal{M}_{1}(\Sigma)$ of elementary moves only change the markings within subsurfaces of genus zero with three or four holes, or within subsurfaces of genus one with one hole. The corresponding operators will be called Moore-Seiberg data. They characterize a tower of representations of the modular groupoid completely. Let us furthermore note that the faces/relations that one needs to define a two-dimensional $\mathrm{CW}$ complex $\mathcal{M}(\Sigma)$ which has as set of edges $\mathcal{M}_{1}(\Sigma)$ turn out to involve only subsurfaces of genus zero with three to five holes, and subsurfaces of genus one with one or two holes. This means that one only needs to verify a finite number of relations to show that a given set of Moore-Seiberg data defines a stable unitary modular functor.

\section{The modular groupoid}

The modular groupoid is the groupoid generated from the natural transformations relating the different markings of a Riemann surface $\Sigma$ [MS][BK1]. A complete set of generators and relations has been determined in $[\mathrm{MS}, \mathrm{BK} 1, \mathrm{FuG}]$. 
Remark 8. The CW complex $\mathcal{M}(\Sigma)$ defined below will be a subcomplex of the complex denoted $\mathcal{M}^{\max }(\Sigma)$ in [BK1], since that reference allowed for cut systems that yield connected components with less than three boundary components, whereas we will exclusively consider cut systems that yield connected components with exactly three holes. All other deviations from [BK1] are due to slightly different conventions in the definition of the generators.

\subsection{Notations and conventions}

We have a unique curve $c(\sigma, e) \in \mathcal{C}^{\prime}$ associated to each edge $e \in \sigma_{\mathbf{1}}$ of the marking graph $\Gamma_{\sigma}$. The trinions $T_{p} \in \mathcal{P}_{\sigma}$ are in one-to-one correspondence with the vertices $p \in \sigma_{\mathcal{o}}$ of $\Gamma_{\sigma}$.

The choice of a distinguished boundary component $c_{p}$ for each trinion $T_{p}, p \in \sigma_{\mathrm{o}}$ will be called the decoration of the marking graph $\Gamma_{\sigma}$. The distinguished boundary component $c_{p}$ will be called outgoing, the other two boundary components of $T_{p}$ incoming. Two useful graphical representation for the decoration are depicted in Figure 10.
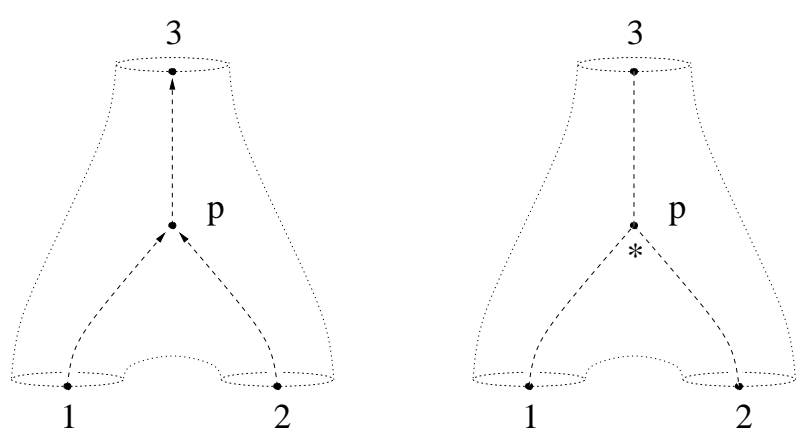

Figure 10: Two representations for the decoration on a marking graph

\subsection{Generators}

The set of edges $\mathcal{M}_{\mathbf{1}}(\Sigma)$ will be given by elementary moves denoted as $(p q), Z_{p}, B_{p}, F_{p q}$ and $S_{p}$. The indices $p, q \in \sigma_{\mathrm{o}}$ specify the relevant trinions within the pants decomposition of $\Sigma$ that is determined by $\sigma$. The move $(p q)$ will simply be the operation in which the labels $p$ and $q$ get exchanged. Graphical representations for the elementary moves $Z_{p}, B_{p}, F_{p q}$ and $S_{p}$ are given in Figures 11-14.

\subsection{Relations}

The relations of $\mathrm{M}(\Sigma)$ correspond to the faces $\varpi \in \mathcal{M}_{2}(\Sigma)$. In the following we will define a set $\mathcal{R}$ of faces which is large enough to make the complex $\mathcal{M}(\Sigma)$ simply connected. A face $\varpi$ in $\mathcal{M}_{2}(\Sigma)$ may be characterized by choosing a chain $\Gamma_{\varpi}=E_{\varpi, n(\varpi)} \circ \cdots \circ E_{\varpi, 1}$, where $E_{\varpi, i} \in \mathcal{M}_{1}(\Sigma)$ for $i=1, \ldots, n(\varpi)$. In order to simplify notation we will generically factorize $\Gamma_{\varpi}$ as $\Gamma_{\varpi}=\Gamma_{\varpi}^{2} \circ \Gamma_{\varpi}^{1}$ and write $\left(\Gamma_{\varpi}^{2}\right)^{-1}=\Gamma_{\varpi}^{1}$ instead of $\Gamma_{\varpi}=\mathrm{id}$.

Locality. Let us introduce the notation $\operatorname{supp}(m)$ by $\operatorname{supp}(m)=\{p\}$ if $m=Z_{p}, B_{p}, S_{p}$, $\operatorname{supp}(m)=\{p, q\}$ if $m=(p q), F_{p q}$ and $\operatorname{supp}\left(m_{\mathbf{2}} \circ m_{\mathbf{1}}\right)=\operatorname{supp}\left(m_{2}\right) \cup \operatorname{supp}\left(m_{1}\right)$. We then 


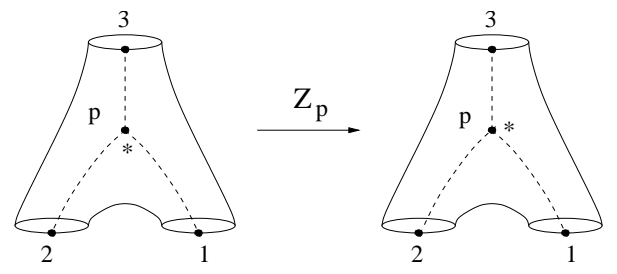

Figure 11: The Z-move

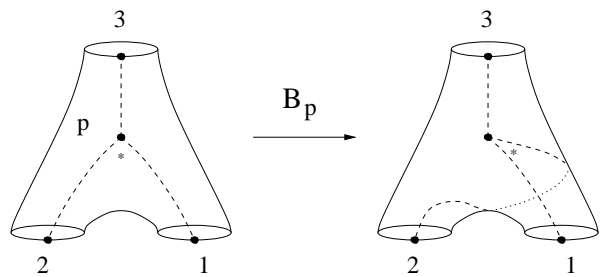

Figure 12: The B-move

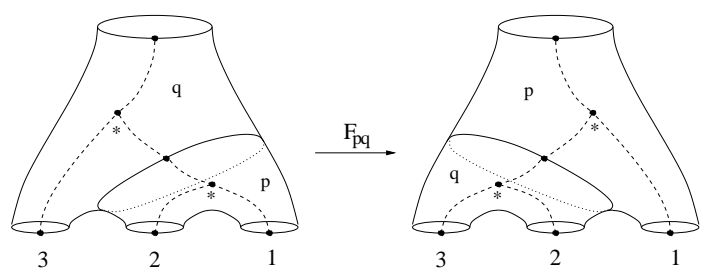

Figure 13: The F-move

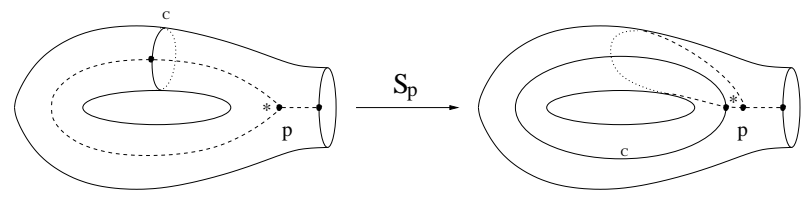

Figure 14: The S-move

have

$$
m_{2} \circ m_{1}=m_{1} \circ m_{2} \quad \text { whenever } \operatorname{supp}\left(m_{1}\right) \cap \operatorname{supp}\left(m_{2}\right)=\emptyset \text {. }
$$

We will list the remaining relations ordered by the topological type of the surfaces on which the relevant graphs can be drawn.

Relations supported on surfaces of genus zero.

$$
\begin{array}{ll}
g=0, \quad s=3: & Z_{p} \circ Z_{p} \circ Z_{p}=\mathrm{id} . \\
& \text { a) } F_{q p} \circ B_{p} \circ F_{p q}=(p q) \circ B_{q} \circ F_{p q} \circ B_{p}, \\
g=0, s=4: & \text { b) } F_{q p} \circ B_{p}^{-1} \circ F_{p q}=(p q) \circ B_{q}^{-1} \circ F_{p q} \circ B_{p}^{-1}, \\
& \text { c) } A_{p q} \circ A_{q p}=(p q) . \\
g=0, s=5: & F_{q r} \circ F_{p r} \circ F_{p q}=F_{p q} \circ F_{q r} .
\end{array}
$$


We have used the abbreviation

$$
A_{p q} \equiv Z_{q}^{-1} \circ F_{p q} \circ Z_{q}^{-1} \circ Z_{p}
$$

In Figures 15 and 16 we have given diagrammatic representations for relations (13.3), b) and (13.4) respectively.

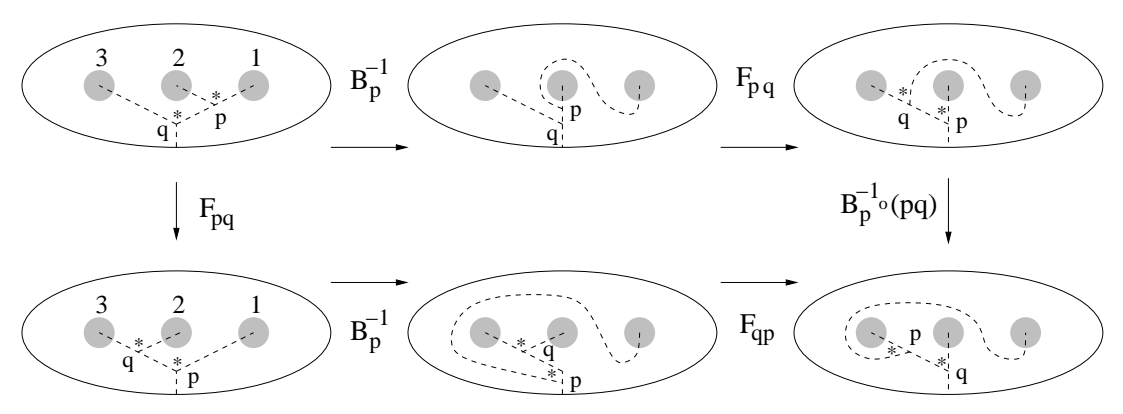

Figure 15: The hexagon relation.

Relations supported on surfaces of genus one. In order to write the relations transparently let us introduce the following composites of the elementary moves.

$$
\begin{array}{ll}
g=0, s=3: & \text { a) } B_{p}^{\prime} \equiv Z_{p}^{-1} \circ B_{p} \circ Z_{p}^{-1}, \\
& \text { b) } T_{p} \equiv Z_{p}^{-1} \circ B_{p} \circ Z_{p} \circ B_{p}, \\
g=0, s=4: & B_{q p} \equiv Z_{q}^{-1} \circ F_{q p}^{-1} \circ B_{q}^{\prime} \circ F_{p q}^{-1} \circ Z_{q}^{-1} \circ(p q), \\
g=1, \quad s=2: & S_{q p} \equiv\left(F_{q p} \circ Z_{q}\right)^{-1} \circ S_{p} \circ\left(F_{q p} \circ Z_{q}\right) .
\end{array}
$$

It is useful to observe that the move $T_{p}$, represents the Dehn twist around the boundary component of the trinion $\mathfrak{t}_{p}$ numbered by $i=1$ in Figure 10. With the help of these definitions we may write the relations supported on surfaces of genus one as follows:

$$
\begin{array}{ll}
g=1, \quad s=1: & \text { a) } S_{p}^{2}=B_{p}^{\prime}, \\
& \text { b) } S_{p} \circ T_{p} \circ S_{p}=T_{p}^{-1} \circ S_{p} \circ T_{p}^{-1} . \\
g=1, \quad s=2: & B_{q p}=S_{q p}^{-1} \circ T_{q}^{-1} T_{p} \circ S_{p q} .
\end{array}
$$

Relation (13.10) is represented diagrammatically in Figure 17.

Theorem 5. The complex $\mathcal{M}(\Sigma)$ is connected and simply connected for any e-surface $\Sigma$.

Proof. The theorem follows easily from [BK1], Theorem 5.1. We noted previously that our complex $\mathcal{M}(\Sigma)$ differs from the complex $\mathcal{M}^{\max }(\Sigma)$ of [BK1] in having a set of vertices which corresponds to decompositions of $\Sigma$ into connected components with exactly three holes. The edges of $\mathcal{M}_{1}^{\max }(\Sigma)$ which correspond to the F-move of [BK1] simply can't appear in $\mathcal{M}_{1}(\Sigma)$. Otherwise the set of edges of $\mathcal{M}(\Sigma)$ coincides with the relevant subset of $\mathcal{M}_{1}^{\max }(\Sigma)$, with the exception that our move $B_{p}$ is a composition of the $B$ - and the $Z$-move of [BK1]. To complete the proof it remains to check that our set of faces is equivalent to the subset of $\mathcal{M}_{2}^{\max }(\Sigma)$ which involves only the vertices $\mathcal{M}_{\mathfrak{o}}(\Sigma)$ of our smaller complex $\mathcal{M}(\Sigma)$. This is a useful exercise. 


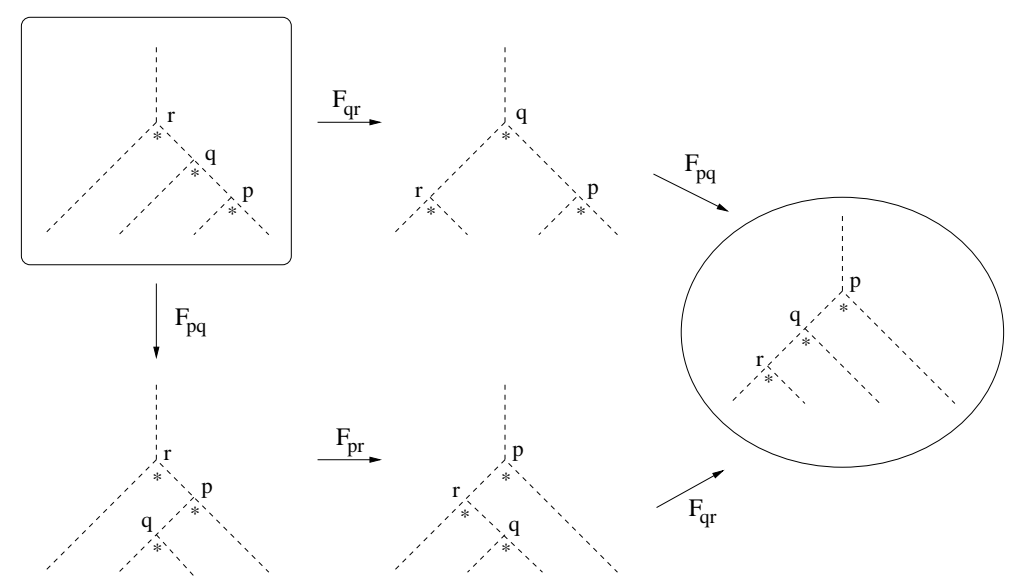

Figure 16: The pentagon identity

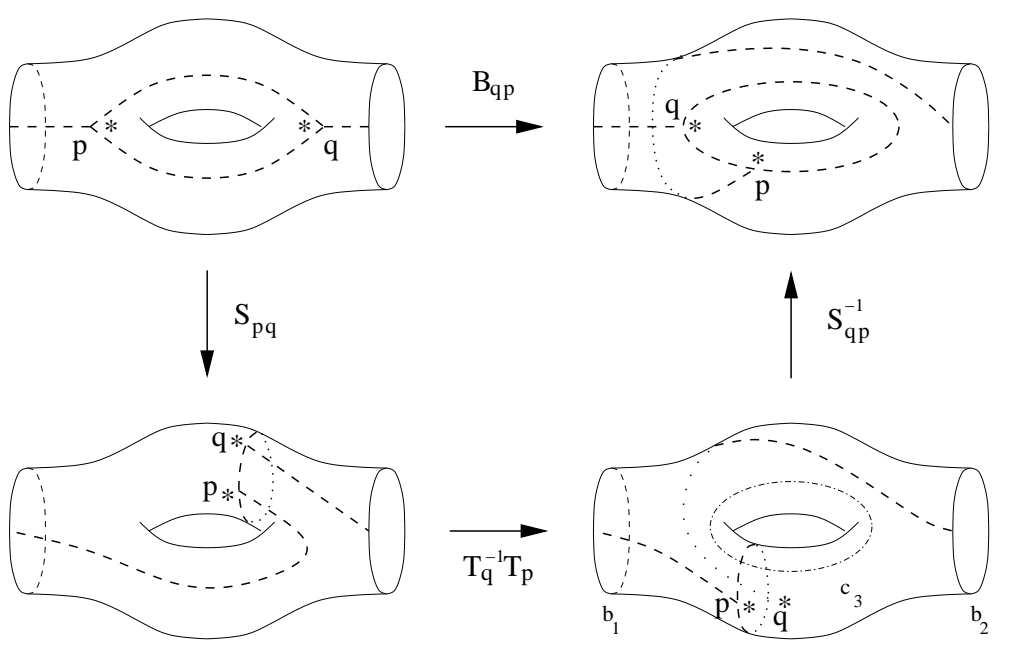

Figure 17: Relation for the two-punctured torus.

Definition 4. Let $\mathcal{M}^{\prime}(\Sigma)$ be the complex which has the same set of vertices as $\mathcal{M}(\Sigma)$, a set of edges given by the moves

$$
(p q), Z_{p}, B_{p}, B_{p}^{\prime}, S_{p}, T_{p}, F_{p q}, A_{p q}, B_{p q}, S_{p q}
$$

defined above, as well as faces given by equations (13.1)-(13.10).

\section{From markings to fat graphs}

A key step in our construction of a stable modular functor from the quantized Teichm uller spaces will be the definition of a distinguished class of fat graphs $\varphi_{\sigma}$ which are associated to the elements $\sigma$ of a certain subset of the set $\mathcal{M}_{\mathcal{o}}(\Sigma)$ of all markings of $\Sigma$.

Definition 5. Let $\mathcal{A}_{\sigma} \subset \mathcal{C}_{\sigma}$ be the set of all curves $c$ which are incoming for both adjacent trinions. We will say that a marking $\sigma$ is admissible iff there is no curve $c \in \mathcal{C}_{\sigma}$ which is outgoing for both adjacent trinions, and if cutting $\Sigma$ along all curves $c \in \mathcal{A}_{\sigma}$ yields connected components all of which have genus zero. The set of all admissible markings will be denoted by $\mathcal{M}_{\mathrm{o}}^{\mathrm{ad}}(\Sigma)$. 


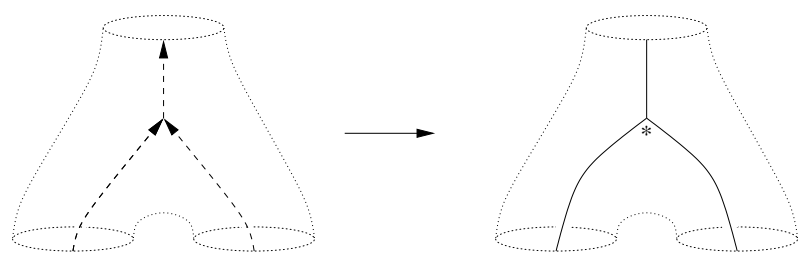

Figure 18: Substitution for a trinion $T$
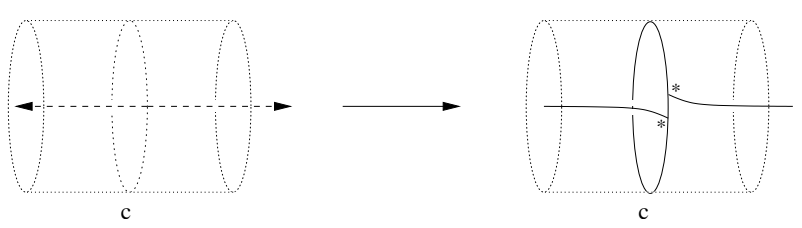

Figure 19: Substitution for an annulus $A$

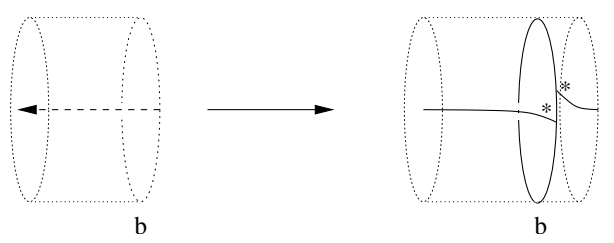

Figure 20: Substitution for a boundary component $B$

To each admissible marking $\sigma$ we may naturally associate a fat graph $\varphi_{\sigma}$ on $\Sigma$ by the following construction. In order to construct the fat graph $\varphi_{\sigma}$, it will be useful to consider a certain refinement of the pants decomposition associated to $\sigma$ which is defined as follows. For each curve $c \in \mathcal{A}_{\sigma}$ let $A_{c}$ be a small annular neighborhood of $c$ which contains $c$ in its interior, and which is bounded by a pair of curves $\left(c^{+}, c^{-}\right)$that are isotopic to $c$. If $c \in A(\Sigma)$ represents a boundary component of $\Sigma$ we may similarly consider an annular subset $B_{c}$ of $\Sigma$ bounded by $c^{+} \equiv c$ and another curve $c^{-}$isotopic to $c$. If $c \in \mathcal{C}_{\sigma}$ belongs to neither of these two classes we will simply set $c^{+} \equiv c \equiv c^{-}$. By cutting along all such curves $c^{ \pm}$we obtain a decomposition of $\Sigma$ into trinions $T_{p}, p \in \sigma_{\mathrm{o}}$ and annuli $A_{c}, c \in \mathcal{A}_{\sigma}$, each equipped with a marking graph. We may then replace the markings on each of these connected components by fat graphs according to Figures 18-20. The re-gluing of trinions $T_{p}, p \in \sigma_{\mathrm{o}}$ and annuli $A_{c}, B_{c}$ to recover the surface $\Sigma$ may then be performed in such a way that the fat graphs on the connected components glue to a fat graph $\varphi_{\sigma}$ on $\Sigma$.

\subsection{The complex $\mathcal{M}^{\text {ad }}(\Sigma)$}

It is natural to consider the complex $\mathcal{M}^{\text {ad }}(\Sigma)$ for which the set of vertices $\mathcal{M}_{\mathrm{o}}^{\text {ad }}(\Sigma)$ is the subset of $\mathcal{M}_{\mathrm{o}}(\Sigma)$ which consists of the admissible markings, and which has a set of edges $\mathcal{M}_{1}^{\text {ad }}(\Sigma)$ given by the subset of $\mathcal{M}_{1}^{\prime}(\Sigma)$ that contains those edges which connect two admissible markings.

Proposition 7. The complex $\mathcal{M}^{\mathrm{ad}}(\Sigma)$ is connected and simply connected. 
Proof. Let us consider two admissible markings $\sigma, \sigma^{\prime} \in \mathcal{M}_{\mathfrak{o}}(\Sigma)$. There exists a path $\varpi$ in $\mathcal{M}(\Sigma)$ which connects $\sigma$ and $\sigma^{\prime}$. This path may be represented as a chain $C_{\varpi}=E_{\varpi, n(\varpi)} \circ \cdots \circ$ $E_{\varpi, 1}, E_{\varpi, i} \in \mathcal{M}_{i}(\Sigma)$ composed out of the moves $Z_{p}, F_{p q}, B_{p}$ and $S_{p}$.

For a given marking $\sigma \in \mathcal{M}_{\mathcal{o}}(\Sigma)$ let $[\sigma]$ be the set of all markings $\sigma^{\prime}$ which differ from $\sigma$ only in the choice of decoration. The moves $Z_{p}$ act transitively on $[\sigma]$. By inserting $Z_{p}$ moves if necessary we may therefore modify $C_{\varpi}$ to a chain $D_{\varpi}$ which takes the form

$$
D_{\varpi}=Z_{\varpi, n(\varpi)} \circ F_{\varpi, n(\varpi)} \circ Z_{\varpi, n(\varpi)-1} \circ \cdots \circ F_{\varpi, 1} \circ Z_{\varpi, 0},
$$

where $Z_{\varpi, i}, i=0, \ldots, n(\varpi)$ are chains composed out of $Z_{p}$-moves only, and the moves $F_{\varpi, n(\varpi)} \in \mathcal{M}_{\mathbf{1}}(\Sigma)$ connect markings $\tilde{\sigma}_{\varpi, i}$ and $\sigma_{\varpi, i}, i=1, \ldots, n(\varpi)$ which are admissible. We clearly must have $\left[\sigma_{\varpi, i+1}\right]=\left[\tilde{\sigma}_{\varpi, i}\right], i=1, \ldots, n(\varpi)-1$ and $\left[\sigma_{\varpi, 1}\right]=[\sigma],\left[\sigma_{\varpi, n(\varpi)}\right]=\left[\sigma^{\prime}\right]$. Connectedness of $\mathcal{M}^{\text {ad }}(\Sigma)$ would follow if the chains $Z_{\varpi, i}, i=0, \ldots, n(\varpi)$ are homotopic to chains $Y_{\varpi, i}$ which represent paths in $\mathcal{M}^{\text {ad }}(\Sigma)$. That this indeed the case follows from the following lemma.

Lemma 8. Assume that $\sigma_{a}, \sigma_{b} \in \mathcal{M}_{\mathrm{o}}^{\mathrm{ad}}(\Sigma)$ satisfy $\sigma_{b} \in\left[\sigma_{a}\right]$. There then exists a path $\varpi_{a b}$ in $\mathcal{M}^{\text {ad }}(\Sigma)$ which connects $\sigma_{a}$ and $\sigma_{b}$.

Sketch of proof. - Let us call a marking $\sigma$ irreducible if it is admissible and if there are no edges $e$ in $\mathcal{A}_{\sigma}$ such that cutting $\Sigma$ along $c(e, \sigma)$ yields two disconnected components. A marking $\sigma$ which is irreducible has only one outgoing external edge. With the help of Lemma 17 in Appendix D it is easy to show that for an admissible graph $\sigma$ there always exists a sequence of $Z_{p}$-moves in $\mathcal{M}_{1}^{\text {ad }}(\Sigma)$ which transforms $\sigma$ to a graph $\sigma^{\prime}$ that is irreducible.

We may and will therefore assume that $\sigma_{a}$ and $\sigma_{b}$ are both irreducible. Using Lemma 17 again allows us to transform $\sigma_{b}$ to a marking $\sigma_{c}$ which is such that the outgoing external edges of $\sigma_{a}$ and $\sigma_{c}$ correspond to the same outgoing boundary component of $\Sigma$.

The graphs $\sigma_{a}$ and $\sigma_{c}$ can finally be connected by a chain which is composed out of moves $B_{p}$ and $F_{q p}$ it only. This follows from the connectedness of $\mathcal{M}\left(\Sigma_{\mathfrak{o}}\right)$ in the case of a surface $\Sigma_{\mathcal{o}}$ of genus zero [MS]. In this way one constructs a sequence of moves that connects $\sigma_{a}$ to $\sigma_{b}$

It remains to prove that $\mathcal{M}^{\text {ad }}(\Sigma)$ is simply connected as well. Let us consider any closed path $\varpi$ in $\mathcal{M}^{\text {ad }}(\Sigma)$. The path $\varpi$ is contractible in $\mathcal{M}^{\prime}(\Sigma)$. The deformation of $\varpi$ to a trivial path may be performed recursively, face by face. The crucial observation to be made is the following one.

Lemma 9. The paths $\varpi$ which represent the boundaries of the faces of $\mathcal{M}_{2}^{\prime}(\Sigma)$ are paths in $\mathcal{M}^{\text {ad }}(\Sigma)$.

Proof. By direct inspection of the relations (13.2)-(13.10).

The Lemma 9 implies that deforming a path $\varpi \in \mathcal{M}^{\text {ad }}(\Sigma)$ by contracting a face in $\mathcal{M}_{2}^{\prime}(\Sigma)$ will produce a path $\varpi^{\prime}$ which still represents a path in $\mathcal{M}^{\text {ad }}(\Sigma)$. It follows that $\varpi$ is contractible in $\mathcal{M}^{\text {ad }}(\Sigma)$. 


\subsection{Separated variables}

We had observed in Section 9 that the Fock variables $z_{e}, e \in \varphi_{1}^{\prime}$ form a set of coordinates for the Teichm üller spaces of surfaces with holes. When considering fat graphs $\varphi_{\sigma}$ associated to a marking $\sigma$ it will be useful to replace the Fock variables $z_{e}, e \in \varphi_{1}^{\prime}$ by an alternative set of coordinates $\left(q_{c}, p_{c}\right), c \in \mathcal{C}_{\sigma}$ for $T_{\varphi}$ which satisfy

$$
\begin{aligned}
& \Omega_{\varphi}\left(p_{c_{1}}, p_{c_{2}}\right)=0=\Omega_{\varphi}\left(q_{c_{1}}, q_{c_{2}}\right), \quad c_{1}, c_{2} \in \mathcal{C}_{\sigma} . \\
& \Omega_{\varphi}\left(p_{c_{1}}, q_{c_{2}}\right)=\delta_{c_{1}, c_{2}}
\end{aligned}
$$

These coordinates are constructed as follows.

For $c \in \mathcal{A}_{\sigma}$ let $A_{c}$ be an annular neighborhood of $c$ such that the part of $\varphi_{\sigma}$ which is contained in $A_{c}$ is isotopic to the model depicted in Figure 21. Let $e_{1}$ and $e_{2}$ be the two edges that are

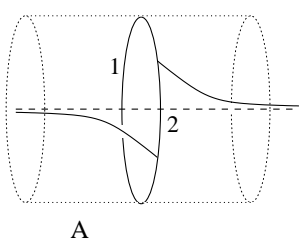

Figure 21: Annulus $A_{c}$ and fat graph $\varphi$ on $A$.

entirely contained in $A_{c}$ with labelling defined by Figure 21 . Out of $z_{e_{1}}$ and $z_{e_{2}}$ we may then define

$$
q_{c} \equiv \frac{1}{2}\left(z_{e_{1}}-z_{e_{2}}\right), \quad p_{c} \equiv-\frac{1}{2}\left(z_{e_{1}}+z_{e_{2}}\right) .
$$

In the case $c \in \mathcal{C}_{\sigma} \backslash \mathcal{A}_{\sigma}$ let us note that there is a unique trinion $T_{c}$ for which the curve $c$ is the outgoing boundary component. The part of $\varphi_{\sigma}$ contained in $T_{c}$ is depicted in Figure 22.

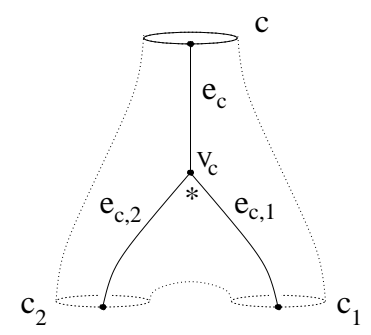

Figure 22: Part of $\varphi_{\sigma}$ contained in $T_{c}$

Let $e_{c, 2}$ and $e_{c, 1}$ be the edges of $\varphi_{\sigma}$ as indicated in Figure 22, and let $c_{\epsilon}, \epsilon=1,2$ be the curves which represent the corresponding boundary components of $T_{p}$. We shall then define

$$
q_{c} \equiv y_{c_{2}}, \quad p_{c} \equiv-y_{c_{1}},
$$

where

$$
y_{c_{\epsilon}} \equiv z_{e_{c, \epsilon}}+\frac{1}{2} f_{c_{\epsilon}}, \quad \epsilon=1,2 .
$$

The same construction yields linear combinations $\left(\hat{p}_{c}, \hat{q}_{c}\right), c \in \mathcal{C}_{\sigma}$ of the Kashaev variables which represent the coordinates $\left(p_{c}, q_{c}\right)$ within $W_{\varphi_{\sigma}}$. Note that our construction of the fat graph $\varphi_{\sigma}$ implies that $T_{c}$ contains a unique vertex $v_{c} \in \varphi_{\sigma, o}$. It turns out that the variables $\hat{q}_{c}, \hat{p}_{c}$ have a simple relation to the Kashaev variables $q_{v_{c}}, p_{v_{c}}$. 
Lemma 10.

$$
\begin{aligned}
& \text { (i) } \hat{q}_{c}=q_{v_{c}}+h_{c_{2}}, \quad \hat{p}_{c}=p_{v_{c}}-h_{c_{1}} . \\
& \text { (ii) } \Omega_{\varphi}\left(p_{c}, q_{c^{\prime}}\right)=\delta_{c, c^{\prime}}, \quad c, c^{\prime} \in \mathcal{C}_{\sigma} .
\end{aligned}
$$

Proof. In order to prove part (i) let us first look at the vertices $v \in \varphi_{\sigma, \mathcal{o}}$ which appear on the graph geodesic homotopic to $c$. The contribution to $y_{c_{\epsilon}}$ of the edges that are incident to $v$ is $\hat{z}_{e_{1}^{\prime}}+\hat{z}_{e_{2}^{\prime}}+\hat{z}_{e_{3}^{\prime}}$ if $e_{i}^{\prime}, i=1,2,3$ are the three edges incident to $v$. It then follows from (7.6) that $y_{c}$ does not depend on both $p_{v}$ and $q_{v}$. What remains are contributions from the vertex $v_{c}$, as well as contributions from the vertices contained in annuli $A_{c^{\prime}}$ which are determined as follows. Note that the homology class $[c]$ can be decomposed as a linear combination of classes $\left[c^{\prime}\right]$ with $c^{\prime} \in \mathcal{A}_{\sigma}$. It is straightforward to check that each $c^{\prime} \in \mathcal{A}_{\sigma}$ which appears in this decomposition yields a contribution $-h_{c^{\prime}}$ to $\hat{f}_{c}$. These contributions sum up to give $-h_{c}$. What remains is the contribution from the vertex $v_{c}$. Part (i) of the lemma now follows easily by recalling the definition 7.6.

Part (ii) of the lemma is a trivial consequence of part (i).

\subsection{Quantized Teichmüller spaces for surfaces with holes}

The coordinates introduced in the previous subsection make it straightforward to modify the discussion of the quantization of Teichm uller spaces from the case of punctured Riemann surfaces (Section 10) to the case of Riemann surfaces with holes.

Bearing in mind that the Fock variables $z_{e}, e \in \varphi_{1}^{\prime}$ are unconstrained in the present case (see Lemma 5) leads us to identify the algebra of functions on the Teichm üller spaces with the algebras of function of the variables $z_{e}, e \in \varphi_{1}^{\prime}$. A convenient set of coordinates is given by the coordinate functions $\left(p_{c}, q_{c}\right), c \in \mathrm{C}_{\sigma}$. Canonical quantization of the Teichm uller spaces is therefore straightforward, and leads to an algebra of operators with generators $\left(\mathrm{p}_{c}, \mathrm{q}_{c}\right), c \in \mathcal{C}_{\sigma}$, which is irreducibly represented (in the sense of Remark 3) on the Hilbert space $\mathcal{H}_{z}(\sigma) \simeq$ $L^{2}\left(\mathbb{R}^{3 g-3+2 s}\right)$. We will normalize the operators $\left(\mathrm{p}_{c}, \mathbf{q}_{c}\right), c \in \mathcal{C}_{\sigma}$, such that

$$
\begin{aligned}
\text { (i) }\left[\mathrm{p}_{c}, \mathrm{q}_{c^{\prime}}\right] & =(2 \pi i)^{-1} \delta_{c c^{\prime}}, \quad c, c^{\prime} \in \mathcal{C}_{\sigma}, \\
\text { (ii) }\left[\mathrm{p}_{c}, \mathrm{p}_{c^{\prime}}\right] & =0=\left[\mathrm{q}_{c}, \mathrm{q}_{c^{\prime}}\right] .
\end{aligned}
$$

Quantization of the Kashaev space $W_{\varphi_{\sigma}}$ produces a reducible representation of the algebra (14.5) which is generated by operators $\left(\hat{\mathrm{q}}_{c}, \hat{\mathbf{p}}_{c}\right)$ associated to the pairs of variables $\left(\hat{q}_{c}, \hat{p}_{c}\right)$. It also yields operators $\mathrm{h}_{c}, c \in H_{1}(\Sigma, \mathbb{R})$ which represent the quantization of the Poisson vector space $H_{\varphi_{\sigma}}$ with basis $h_{c}, c \in H_{1}(\Sigma, \mathbb{R})$. The algebra generated by the $\mathrm{h}_{c}, c \in H_{1}(\Sigma, \mathbb{R})$ has a center generated by the $\mathrm{h}_{c}, c \in B(\Sigma)$. Following the discussion at the beginning of Subsection 10.3 one constructs a representation of this algebra on the space

$$
\mathcal{H}_{h}(\sigma) \equiv \int_{B^{\prime}(\Sigma)} d \mathfrak{f} \mathcal{H}_{h, \mathfrak{f}}(\sigma)
$$

where $\mathcal{H}_{h, f}(\sigma) \simeq L^{2}\left(\mathbb{R}^{g}\right)$ is an irreducible representation of the algebra $i\left[h_{c_{2}}, h_{c_{1}}\right]=b^{2} \mathrm{I}\left(c_{2}, c_{1}\right)$ for $c_{2}, c_{\mathbf{1}} \in H_{1}\left(\Sigma_{\mathrm{cl}}, \mathbb{R}\right)$.

The following Proposition 8 describes how the quantized Teichm uller spaces are related to the quantized Kashaev space. 
Proposition 8. There exists a unitary operator $\mathrm{I}_{\sigma}$,

$$
\mathrm{I}_{\sigma}: \mathcal{K}\left(\varphi_{\sigma}\right) \rightarrow \mathcal{H}_{z}(\sigma) \otimes \mathcal{H}_{h}(\sigma)
$$

such that

$$
\begin{aligned}
& \mathrm{I}_{\sigma} \cdot \hat{\mathrm{p}}_{c} \cdot \mathrm{I}_{\sigma}^{-1}=\mathrm{p}_{c} \otimes 1, \\
& \mathrm{I}_{\sigma} \cdot \hat{\mathrm{q}}_{c} \cdot \mathrm{I}_{\sigma}^{-1}=\mathrm{q}_{c} \otimes 1,
\end{aligned} \quad \text { and } \quad \mathrm{I}_{\sigma} \cdot \mathrm{h}_{c} \cdot \mathrm{I}_{\sigma}^{-1}=1 \otimes \mathrm{h}_{c}^{\prime},
$$

for any $e \in \sigma_{1}$ and $c \in H_{1}(\Sigma, \mathbb{R})$, respectively.

Let us recall that a move $m \in\left[\tau_{m}, \sigma_{m}\right], m \in \mathcal{M}_{\mathbf{1}}(\Sigma)$ is admissible if both $\tau_{m}$ and $\sigma_{m}$ are admissible. Given an element $m \in \mathcal{M}_{1}^{\text {ad }}(\Sigma)$ it is natural to consider the corresponding fat graphs $\theta_{m} \equiv \varphi_{\tau_{m}}, \varphi_{m} \equiv \varphi_{\sigma_{m}}$ on $\Sigma$, to pick a path $\pi_{m} \in\left[\theta_{m}, \varphi_{m}\right]$ and consider the operator $\mathrm{u}(m) \equiv \mathrm{u}\left(\pi_{m}\right)$. The reduction to the quantized Teichm üller spaces proceeds as in Subsection 11.4. By multiplying the operators $\mathrm{I}_{\sigma} \cdot \mathrm{u}(m) \cdot \mathrm{I}_{\sigma}^{-1}$ with suitably chosen operators $\mathrm{H}(m)$ one gets operators $\mathrm{v}(m)$ which factorize as $\mathrm{v}(m)=\mathrm{w}(m) \otimes 1$. The resulting operators $\mathrm{w}(m)$ : $\mathcal{H}_{z}\left(\varphi_{m}\right) \rightarrow \mathcal{H}_{z}\left(\theta_{m}\right)$ will then generate a unitary projective representation of the path groupoid of $\mathcal{M}^{\text {ad }}(\Sigma)$.

\section{Geodesic length operators}

Of fundamental importance for us will be to define and study quantum analogs of the geodesic length functions on the Teichm uller spaces, the geodesic length operators.

\subsection{Overview}

When trying to define operators which represent the geodesic length functions one has to face the following difficulty: The classical expression for $L_{\varphi, c} \equiv 2 \cosh \frac{1}{2} l_{c}$ as given by formula 8.6 is a linear combination of monomials in the variables $e^{ \pm \frac{z_{e}}{2}}$ of a very particular form,

$$
L_{\varphi, c}=\sum_{\tau \in \mathcal{F}} C_{\varphi, c}(\tau) e^{x(\tau)}, \quad x(\tau) \equiv \sum_{e \in \varphi_{1}} \tau(e) z_{e}
$$

where the summation is taken over the space $\mathcal{F}$ of all maps $\varphi_{1} \ni e \rightarrow \tau(e) \in \frac{1}{2} \mathbb{Z}$. The coefficients $C_{\varphi, c}(\tau)$ are positive integers, and non-vanishing for a finite number of $\tau \in \mathcal{F}$ only.

In the quantum case one is interested in the definition of length operators $\mathrm{L}_{\varphi, c}$ which should be representable by expressions similar to (15.1),

$$
\mathrm{L}_{\varphi, c}=\sum_{\tau \in \mathcal{F}} C_{\varphi, c}^{b}(\tau) e^{\times(\tau)}, \quad \mathrm{x}(\tau) \equiv \sum_{e \in \varphi_{1}} \tau(e) \mathrm{z}_{e}
$$

The following properties seem to be indispensable if one wants to interpret an operator of the general form (15.2) as the quantum counterpart of the functions $L_{\varphi, c}=2 \cosh \frac{1}{2} l_{c}$ :

(a) Spectrum: $\mathrm{L}_{\varphi, c}$ is self-adjoint. The spectrum of $\mathrm{L}_{\varphi, c}$ is simple and equal to $[2, \infty)$. This is necessary and sufficient for the existence of an operator $\mathrm{I}_{\varphi, c}$ - the geodesic length operator - such that $\mathrm{L}_{\varphi, c}=2 \cosh \frac{1}{2} \mathrm{I}_{c}$. 
(b) Commutativity:

$$
\left[\mathrm{L}_{\varphi, c}, \mathrm{~L}_{\varphi, c^{\prime}}\right]=0 \text { if } c \cap c^{\prime}=\emptyset .
$$

(c) Mapping class group invariance:

$$
\mathrm{a}_{\mu}\left(\mathrm{L}_{\varphi, c}\right)=\mathrm{L}_{\mu . \varphi, c}, \quad \mathrm{a}_{\mu} \equiv \mathrm{a}_{[\mu . \varphi, \varphi]}, \quad \text { for all } \mu \in \operatorname{MC}(\Sigma) .
$$

(d) Classical limit: The coefficients $C_{\varphi, c}^{b}(\tau)$ which appear in (15.2) should be deformations of the classical coefficients $C_{\varphi, c}(\tau)$ in the sense that

$$
\lim _{b \rightarrow 0} C_{\varphi, c}^{b}(\tau)=C_{\varphi, c}(\tau)
$$

Length operators were first defined and studied in the pioneering work [CF2]. It was observed in [CF2] that the necessary deformation of the coefficients $C_{\varphi, c}^{b}(\tau)$ is indeed nontrivial in general. However, a full proof that the length operators introduced in [CF2] fulfil the requirements (a) and (c) does not seem to be available yet. We will therefore present an alternative approach to this problem, which will allow us to define length operators that satisfy (a)-(d) in full generality.

\subsection{Construction of the length operators}

Our construction of the length operators will proceed in two steps. First, we will construct length operators $\mathrm{L}_{\sigma, c}$ in the case that the fat graph $\varphi$ under consideration equals $\varphi_{\sigma}$. This will facilitate the verification of the properties (a)-(d) formulated above. In order to define the length operators $\mathrm{L}_{\varphi, c}$ in the general case we shall then simply pick any marking $\sigma$ such that the given curve $c$ is contained in the cut system $\mathcal{C}_{\sigma}$, and define

$$
\mathrm{L}_{\varphi, c} \equiv \mathrm{a}_{\left[\varphi, \varphi_{\sigma}\right]}\left(\mathrm{L}_{\sigma, c}\right) \text {. }
$$

Independence of this construction from the choice of $\sigma$ will follow from Theorem 6 below. Definition (15.3) reduces the proof of properties (a), (b) to the proof of the corresponding statements for the length operators $\mathrm{L}_{\sigma, c}$ which will be given below. Property (c) follows from $\mathrm{a}_{[\mu . \varphi, \varphi]} \circ \mathrm{a}_{\left[\varphi, \varphi_{\sigma}\right]}=\mathrm{a}_{\left[\mu . \varphi, \varphi_{\sigma}\right]}$.

In order to prepare for our construction of length operators it is useful us recall the construction of the fat graph $\varphi_{\sigma}$ in section 14 .

\section{Definition 6.}

Case $c \in \mathcal{A}_{\sigma}:$ Let $A$ be an annular neighborhood of $c$ such that the part of $\varphi_{\sigma}$ which is contained in $A$ is isotopic to the model depicted in Figure 21. We will then define

$$
\mathrm{L}_{\varphi, c} \equiv e^{-2 \pi b \mathbf{q}_{c}}+2 \cosh 2 \pi b \mathbf{p}_{c} .
$$

Case $c \notin \mathcal{A}_{\sigma}$ : If a curve $c$ is not contained in $\mathcal{A}_{\sigma}$, it is necessarily the outgoing boundary component of a trinion $T_{p}$ (cf. Figure 18). Let $c_{\epsilon}, \epsilon=1,2$ be the curves which represent the incoming boundary components of $T_{p}$ as indicated in Figure 22. Given that $\mathrm{L}_{\sigma ; c_{i}}, i=1,2$ are already defined we will define $\mathrm{L}_{\sigma ; c}$ by

$$
\mathrm{L}_{\sigma, c}=2 \cosh \left(\mathrm{y}_{c_{2}}+\mathrm{y}_{c_{1}}\right)+e^{-\mathrm{y}_{c_{2}}} \mathrm{~L}_{\sigma, c_{1}}+e^{\mathrm{y}_{c_{1}}} \mathrm{~L}_{\sigma, c_{2}}+e^{\mathrm{y}_{c_{1}}-\mathrm{y}_{c_{2}}}
$$

where $\mathrm{y}_{c_{\epsilon}}, \epsilon=1,2$ are defined as $\mathrm{y}_{c_{2}}=2 \pi b \mathrm{q}_{c}, \mathrm{y}_{c_{1}}=-2 \pi b \mathrm{p}_{c}$. 
It is easy to see that this recursively defines length operators for all remaining $c \in \mathcal{C}_{\sigma}$.

Remark 9. Let us note that (14.5) implies that $\left[\mathrm{y}_{c_{\epsilon}}, \mathrm{L}_{\sigma, c_{\epsilon^{\prime}}}\right]=0$ for $\epsilon, \epsilon^{\prime} \in\{1,2\}$. We therefore do not have an issue of operator ordering in (15.5).

Proposition 9. The length operators $\mathrm{L}_{\sigma, c}, \mathrm{~L}_{\sigma, c^{\prime}}$ associated to different curves $c, c^{\prime} \in \mathcal{C}_{\sigma}$ commute with each other.

Proof. Let us recall that cutting the surface $\Sigma$ along all of the curves $c \in \mathcal{A}_{\sigma}$, yields a set of connected components which all have genus zero. For a given curve $c \in \mathcal{C}_{\sigma} \backslash \mathcal{A}_{\sigma}$ let $\Sigma_{c}$ be the connected component which contains $c$. Cutting $\Sigma_{c}$ along $c$ produces two connected components. The component which has $c$ as its outgoing boundary component will be denoted $\Sigma_{c}^{\prime}$. It follows from Definition 6 that $\mathrm{L}_{\sigma, c}$ is an operator function of the operators $\mathrm{p}_{d}$ and $\mathrm{q}_{d}$, where $d \in \mathcal{C}_{\sigma}$ is contained in $\Sigma_{c}^{\prime}$. The claim therefore follows immediately from (14.5) if $\Sigma_{c}^{\prime}$ and $\Sigma_{c^{\prime}}^{\prime}$ are disjoint.

Otherwise we have the situation that one of $\Sigma_{c}^{\prime}, \Sigma_{c^{\prime}}^{\prime}$, say $\Sigma_{c^{\prime}}^{\prime}$ is a subsurface of the other. The crucial point to observe is that the resulting expression for $\mathrm{L}_{\sigma, c}$ depends on the variables $\mathrm{p}_{d}$ and $\mathrm{q}_{d}$ associated to the subsurface $\Sigma_{c^{\prime}}^{\prime}$ exclusively via $\mathrm{L}_{\sigma, c^{\prime}}$. The claim therefore again follows from (14.5).

The following theorem expresses the consistency of our definition with the automorphisms induced by a change of the marking $\sigma$.

Theorem 6. For a given curve $c$ let $\sigma_{i}, i=1,2$ be markings such that $c$ is contained in both cut systems $\mathcal{C}_{\sigma_{1}}$ and $\mathcal{C}_{\sigma_{2}}$. We then have

$$
\mathrm{a}_{\left[\varphi_{\sigma_{2}}, \varphi_{\sigma_{1}}\right]}\left(\mathrm{L}_{\sigma_{1}, c}\right)=\mathrm{L}_{\sigma_{2}, c}
$$

On the proof of Theorem 6: The description of the modular groupoid in terms of generators and relations given in section 13 reduces the proof of Theorem 6 to the case that $\sigma_{2}$ and $\sigma_{1}$ are connected by one of the elementary moves $m$ defined in section 13.2. In order to reduce the proof of Theorem 6 to a finite number of verifications one would need to have simple standard choices for the paths $\pi_{m} \in\left[\varphi_{\sigma_{2}}, \varphi_{\sigma_{1}}\right]$ for all elementary moves $m$. Existence of such standard paths $\pi_{m}$ turns out to be nontrivial, though. The task to find suitable paths $\pi_{m}$ is particularly simple for a subclass of moves $m$ which is defined as follows.

\section{Definition 7.}

(i) Let $\pi \in\left[\varphi^{\prime}, \varphi\right]$ be a path in the complex $\mathcal{P} t(\Sigma)$ which is described by a sequence $S_{\pi} \equiv$ $\left(\varphi^{\prime} \equiv \varphi_{n}, \ldots, \varphi_{1} \equiv \varphi\right)$ of fat graphs such that consecutive elements of $S_{\pi}$ are connected by edges in $\mathcal{P} t_{1}(\Sigma)$. We will say that $\pi \in\left[\varphi^{\prime}, \varphi\right]$ is realized locally in a subsurface $\Sigma^{\prime} \hookrightarrow \Sigma$ if the restrictions of $\varphi_{i}$ to $\Sigma \backslash \Sigma^{\prime}$ coincide for all $i=1, \ldots, n$.

(ii) We will say that the move $m=\left[\tau_{m}, \sigma_{m}\right] \in \mathcal{M}_{1}^{\prime}(\Sigma)$ can be realized locally if there exists a path $\pi \in\left[\theta_{m}, \varphi_{m}\right]$ in the complex $\mathcal{P} t(\Sigma)$ that is realized locally in $\Sigma_{m}$ in the sense of $(i)$.

For moves $m=\left[\tau_{m}, \sigma_{m}\right] \in \mathcal{M}_{\mathbf{1}}(\Sigma)^{\prime}$ that can be realized locally we may choose essentially the same path $\pi_{m} \in\left[\theta_{m}, \varphi_{m}\right]$ for all surfaces $\Sigma$ into which $\Sigma_{m}$ can be embedded. It is then crucial to observe the following fact 
Proposition 10. If $m \in \mathcal{M}_{1}^{\prime}(\Sigma)$ is admissible, but can not be realized locally, there always exists a path $\varpi_{m}$ which is $(i)$ homotopic to $m$ within $\mathcal{M}^{\text {ad }}(\Sigma)$, and (ii) takes the form

$$
\varpi_{m}=Y_{m} \circ m \circ Y_{m}^{-1}
$$

where $Y_{m}$ is a chain composed out of $Z_{p}$-moves and $F_{p q}$-moves which can all be realized locally.

The proof of Proposition 10 is given in Appendix D. It therefore suffices to prove Theorem 6 in the case that $\sigma_{2}$ and $\sigma_{1}$ are connected by any elementary move $m$ that can be realized locally. This amounts to a finite number of verifications which can be carried out by straightforward, but tedious calculations. Some details are given in the Appendix E.

\subsection{Spectrum}

Theorem 7. The spectrum of $\mathrm{L}_{\sigma, c}$ is simple and equal to $[2, \infty)$.

Proof. To begin with, let us consider the following simple model for the length operators:

$$
\mathbf{L} \equiv 2 \cosh 2 \pi b \mathrm{p}+e^{-2 \pi b q}
$$

where $\mathrm{p}, \mathrm{q}$ are operators on $L^{2}(\mathbb{R})$ which satisfy the commutation relations $[\mathrm{p}, \mathrm{q}]=(2 \pi i)^{-1}$.

A basic fact is that $L$ is self-adjoint. Indeed, being a sum of two positive self-adjoint operators $L$ is self-adjoint on the intersection of the domains of the summands. The main spectral properties of this operator are summarized in the following proposition.

Proposition 11. - $[\mathrm{Ka} 4]-$

(i) We have $\operatorname{Spec}(\mathrm{L})=(2, \infty)$.

(ii) The spectrum of $\mathrm{L}$ in $L^{2}(\mathbb{R})$ is simple.

Validity of Theorem 7 in the case $c \in \mathcal{A}_{\sigma}$ is a direct consequence of Proposition 11. It remains to treat the case $c \notin \mathcal{A}_{\sigma}$. We will keep the notations introduced in Definition 6 . The main ingredient will be an operator $\mathrm{C}_{\sigma, c}: \mathcal{H}(\sigma) \rightarrow \mathcal{H}(\sigma)$ which maps all length operators $\mathrm{L}_{\sigma, c}$ to the simple standard form $\mathrm{L}_{\sigma, c}^{\mathrm{st}}$,

$$
\mathrm{L}_{\sigma, c}^{\mathrm{st}}=2 \cosh 2 \pi b \mathrm{p}_{c}+e^{-2 \pi b \mathbf{q}_{c}}
$$

in the sense that the following commutation relations are satisfied:

$$
\mathrm{C}_{\sigma, c} \cdot \mathrm{L}_{\sigma, c}=\mathrm{L}_{\sigma, c}^{\mathrm{st}} \cdot \mathrm{C}_{\sigma, c} .
$$

Definition 8. Let the unitary operator $\mathrm{C}_{\sigma, c}$ be defined by

$$
\mathrm{C}_{\sigma, c}^{-1}=e_{b}\left(\mathrm{q}_{c}-\mathrm{s}_{2}\right) \frac{s_{b}\left(\mathrm{~s}_{1}-\mathrm{p}_{c}\right)}{s_{b}\left(\mathrm{~s}_{1}+\mathrm{p}_{c}\right)} e^{2 \pi i \mathrm{~s}_{2} \mathrm{q}_{c}},
$$

where

$$
\mathrm{s}_{\epsilon}=(2 \pi b)^{-1} \operatorname{arcosh} \frac{1}{2} \mathrm{~L}_{\sigma, c_{\epsilon}} \quad \epsilon=1,2,
$$

and the special function $s_{b}(x)$ is a close relative of $e_{b}(x)$ defined in the Appendix $A$. 
Lemma 11. The unitary operator $\mathrm{C}_{\sigma, p}$ satisfies (15.10).

Proof. Proposition 11 is proven by means of a direct calculation using the explicit form of $\mathrm{L}_{\sigma, p}$ given in (15.5) and the functional equations (A.3) and (A.9).

The proof of Theorem 7 is thereby reduced to Proposition 11.

\subsection{Relation with the Dehn twist generator}

To round off the picture we shall now discuss, following [Ka3, Ka4], the relation between the length operators $\mathrm{L}_{\varphi, c}$ and the operator $\mathrm{D}_{\varphi, c}$ which represents the Dehn twist $D_{c}$ along $c$.

A closed curve $c$ will be called a curve of simple type if the the connected components of $\Sigma \backslash c$ all have more than one boundary component. It is not hard to see (using the construction in Section 14, for example) that for curves of simple type there always exists a fat graph $\varphi$ and an annular neighborhood $A_{c}$ of $c$ in which $\varphi$ takes the form depicted in Figure 23.

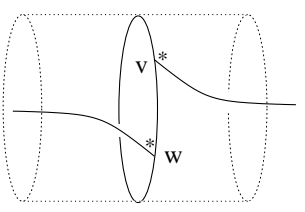

Figure 23: Annulus $A_{c}$ and fat graph $\varphi$ on $A$.

Lemma 12. Let $\Sigma$ be a surface with genus $g$ and s holes. The pure mapping class group $\mathrm{MC}(\Sigma)$ is generated by the Dehn twists along geodesics of simple type.

Proof. For $g \geq 2$ it is known that the Dehn twists along non-separating closed curves suffice to generate the mapping class group $\mathrm{MC}(\Sigma)[\mathrm{Ge}]$. Closed curves which are non-separating are always of simple type.

For $g=0$ any closed curve is of simple type. In the remaining case $g=1$ one may note that the only closed curve $c$ which is not of simple type is the one which separates a one-holed torus from the rest of $\Sigma$. It is then well-known that the Dehn twists along $a$ - and $b$-cycles of the one-holed torus generate the Dehn-twist along $c$. If we supplement these generators by the Dehn twists along the remaining closed curves (which are all of simple type), we get a complete system of generators for $\mathrm{MC}(\Sigma)[\mathrm{Ge}]$.

We may therefore use a fat graph which in an annular neighborhood of $c$ takes the form depicted in Figure 23. It is easy to see that the action of the Dehn twist $D_{c}$ on the fat graph $\varphi$ can be undone by a single flip $\omega_{v w}$. As the representative $\mathrm{F}_{\varphi, c}$ for the Dehn twist $D_{c}$ on $\mathcal{K}(\varphi)$ we may therefore choose $\mathrm{F}_{\varphi, c}=\mathrm{T}_{v w}$.

The operator $\mathrm{h}_{\varphi, c}$ associated to the homology cycle $c$ is $\mathrm{h}_{\varphi, c} \equiv \frac{1}{2}\left(\mathrm{p}_{v}+\mathrm{q}_{w}\right)$. It is not very difficult to verify that the operator

$$
\mathrm{D}_{\varphi, c}=\zeta^{-6} \exp \left(2 \pi i \mathrm{~h}_{\varphi, c}^{2}\right) \mathrm{F}_{\varphi, c} .
$$

commutes with all $\mathrm{h}_{c}, c \in H_{1}(\Sigma, \mathbb{R})$. The prefactor $\zeta^{-6}, \zeta=e^{\pi i b^{2} / 3}$ was inserted to define a convenient normalization. We then have the following result. 
Proposition 12. [Ka4] $-\mathrm{D}_{c}$ coincides with the following function of the length operator $\mathrm{I}_{c}$ :

$$
\mathrm{D}_{c}=\zeta^{-6} \exp \left(i \frac{\mathrm{I}_{c}^{2}}{8 \pi b^{2}}\right) .
$$

This should be compared with the classical result that the geodesic length functions are the Hamiltonian generators of the Fenchel-Nielsen twist flow, which reproduces the Dehn twist for a twist angle of $2 \pi$.

\section{Passage to the length representation}

\subsection{The length representation}

Our aim is to define a representation for $\mathcal{H}(\Sigma)$ which is such that the length operators associated to a cut system are all realized as multiplication operators. For a surface $\Sigma$ and a marking $\sigma$ on $\Sigma$ let

$$
\mathcal{H}_{\mathrm{L}}(\sigma) \equiv \mathcal{K}_{\mathrm{sp}}^{\otimes \sigma_{1}}
$$

where $\mathcal{K}_{\mathrm{sp}} \simeq L^{2}\left(\mathbb{R}_{+}, \eta\right)$, and $\eta$ is the spectral measure of the operator $\mathrm{I}_{\mathrm{A}}=2 \operatorname{arcosh} \frac{1}{2} \mathrm{~L}_{\mathrm{A}}$. The numbering of the edges associated to $\sigma$ defines canonical isomorphisms

$$
\mathcal{H}_{\mathrm{L}}(\sigma) \simeq \mathcal{H}_{\mathrm{L}}(\Sigma) \equiv L^{2}\left(\mathfrak{L}, d \eta_{\mathfrak{L}}\right)
$$

where $\mathfrak{L} \simeq \mathbb{R}_{+}^{3 g-3+2 s}$ and $d \eta_{\mathfrak{L}}=d \eta\left(l_{1}\right) \wedge \cdots \wedge d \eta\left(l_{3 g-3+2 s}\right)$ is the corresponding product measure. For $e \in \sigma_{\mathrm{I}}$ and $f: \mathbb{R}^{+} \rightarrow \mathbb{C}$ let us define the multiplication operator $\mathrm{m}_{e}[f]$ by

$$
\left(\mathrm{m}_{e}[f] \Psi\right)\left(\lambda_{\sigma}\right) \equiv f\left(l_{e}\right) \Psi\left(\lambda_{\sigma}\right)
$$

The operator $\mathrm{m}_{e}[f]$ will represent a bounded operator $\mathrm{m}_{e}[f]: \mathcal{H}_{\mathrm{L}}(\sigma) \rightarrow \mathcal{H}_{\mathrm{L}}(\sigma)$ iff $f \in$ $L^{\infty}\left(\mathbb{R}^{+}, d \eta\right)$.

For the rest of this section let us consider an admissible marking $\sigma$ and the associated fat graph $\varphi_{\sigma}$. The Definition 6 yields a family of mutually commuting length operators $\mathrm{L}_{\sigma, c}$ associated to the curves $c$ which constitute the cut sytem $\mathcal{C}_{\sigma}$. It follows from the spectral theorem ${ }^{9}$ for the family $\left\{\mathrm{L}_{e} ; e \in \sigma_{1}\right\}$ of self-adjoint operators that there exists a unitary operator

$$
\mathrm{D}(\sigma): \mathcal{H}_{z}(\sigma) \longrightarrow \mathcal{H}_{\mathrm{L}}(\sigma)
$$

that diagonalizes the set of length operators $\mathrm{L}_{\sigma, e}, e \in \sigma_{1}$ in the sense that

$$
\mathrm{D}(\sigma) \cdot \mathrm{L}_{\sigma, e}=\mathrm{m}_{e}\left[2 \cosh \frac{1}{2} l\right] \cdot \mathrm{D}(\sigma)
$$

Our aim for the rest of this section will be to give a recursive construction for such an operator $\mathrm{D}(\sigma)$ in terms of operators $\mathrm{C}_{p}$ which are associated to the vertices $p \in \sigma_{\mathrm{o}}$ of $\sigma$.

\footnotetext{
${ }^{9}$ See Appendix B for the precise statement.
} 


\subsection{Construction of the operator $\mathrm{D}(\sigma)$}

The main ingredient will be an operator $\mathrm{C}_{\sigma}: \mathcal{H}(\sigma) \rightarrow \mathcal{H}(\sigma)$ which maps all length operators $\mathrm{L}_{\sigma, c}$ to the simple standard form $\mathrm{L}_{c}^{\text {st }}$,

$$
\mathrm{L}_{\sigma, c}^{\mathrm{st}}=e^{-2 \pi b \mathbf{q}_{c}}+2 \cosh 2 \pi b \mathbf{p}_{c} .
$$

The operators $C_{\sigma, c}$ that were defined in Definition 8 solve this task locally for every curve $c \in \mathcal{C}_{\sigma} \backslash \mathcal{A}_{\sigma}$. If the trinion $T_{p}$ has $c_{p}$ as the outgoing boundary component we will define

$$
\mathrm{C}_{\sigma, p} \equiv \mathrm{C}_{\sigma, c_{p}}
$$

We are now ready to define

$$
\mathrm{C}_{\sigma} \equiv \prod_{p \in \sigma_{\circ}} \mathrm{C}_{\sigma, p}
$$

Let us note that we do not have to specify the order in which the operators $C_{\sigma, p}$ appear thanks to the following lemma.

Lemma 13. We have $\mathrm{C}_{\sigma, p} \mathrm{C}_{\sigma, q}=\mathrm{C}_{\sigma, q} \mathrm{C}_{\sigma, p}$.

Proof. This follows from Proposition 9 and Definition 6, keeping in mind (14.5).

In order to construct the sought-for operator $\mathrm{D}(\sigma)$ it now remains to map the length operators $\mathrm{L}_{\sigma, e}^{\text {st }}$ to multiplication operators. Proposition 11 ensures existence of an operator

$$
\mathrm{d}_{\sigma, e}: L^{2}(\mathbb{R}) \rightarrow \mathcal{K}_{\mathrm{sp}} \quad \text { such that } \quad \mathrm{d}_{\sigma, e} \cdot \mathrm{L}_{e}^{\mathrm{st}}=\mathrm{m}_{e}\left[2 \cosh \frac{1}{2} l\right] \cdot \mathrm{d}_{\sigma, e} .
$$

Out of $\mathrm{E}_{\sigma}$ we may finally define the operator $\mathrm{D}(\sigma)$ as

$$
\mathrm{D}(\sigma) \equiv \mathrm{d}_{\sigma} \cdot \mathrm{C}_{\sigma}, \quad \mathrm{d}_{\sigma} \equiv \bigotimes_{e \in \sigma_{1}} \mathrm{~d}_{\sigma, e},
$$

where $\mathrm{I}_{\sigma}$ is the operator introduced in Proposition 4. It is straightforward to verify that the operator $\mathrm{D}(\sigma)$ indeed satisfies the desired property (16.4).

\section{Realization of $\mathrm{M}(\Sigma)$}

\subsection{Two constructions for the generators}

Our aim is to define operators $\mathrm{U}(m)$ associated to the edges $m \in \mathcal{M}_{\mathbf{1}}(\Sigma)$. We will give two constructions for these operators, each of which makes certain properties manifest. The proof of the equivalence of these two constructions will be the main difficulty that we will have to deal with.

The first construction. Let us recall that a move $m \in\left[\tau_{m}, \sigma_{m}\right], m \in \mathcal{M}_{\mathbf{1}}(\Sigma)$ is admissible if both $\tau_{m}$ and $\sigma_{m}$ are admissible. Given an element $m \in \mathcal{M}_{1}^{\text {ad }}(\Sigma)$ it is natural to consider the corresponding fat graphs $\theta_{m} \equiv \varphi_{\tau_{m}}, \varphi_{m} \equiv \varphi_{\sigma_{m}}$ on $\Sigma$, to pick a path $\pi_{m} \in\left[\theta_{m}, \varphi_{m}\right]$ and define operators $\widetilde{\mathrm{U}}(m): \mathcal{H}_{\mathrm{L}}\left(\sigma_{m}\right) \rightarrow \mathcal{H}_{\mathrm{L}}\left(\tau_{m}\right)$ as

$$
\widetilde{\mathrm{U}}(m) \equiv \mathrm{D}\left(\tau_{m}\right) \cdot \mathrm{u}\left(\pi_{m}\right) \cdot \mathrm{D}\left(\sigma_{m}\right)^{\dagger} \text {. }
$$


The second construction. We note that for all $m \in \mathcal{M}_{1}(\Sigma)$ the markings $\tau_{m}$ and $\sigma_{m}$ will coincide outside of a subsurface $\Sigma_{m} \hookrightarrow \Sigma$. Let us therefore consider the restrictions $\tau_{m}^{\prime}$ and $\sigma_{m}^{\prime}$ of $\tau_{m}$ and $\sigma_{m}$ to $\Sigma_{m}$ respectively. Admissibility of $\tau_{m}^{\prime}$ and $\sigma_{m}^{\prime}$ is obvious for $m \in \mathcal{M}_{\mathbf{1}}(\Sigma)$, allowing us to use the first construction in order to define an operator

$$
\mathrm{U}^{\prime}(m): \mathcal{H}_{\mathrm{L}}\left(\sigma_{m}^{\prime}\right) \rightarrow \mathcal{H}_{\mathrm{L}}\left(\tau_{m}^{\prime}\right)
$$

Out of $\mathbf{U}^{\prime}(m)$ we may then construct the sought-for operator $\mathbf{U}(m)$ by acting with $\mathbf{U}^{\prime}(m)$ nontrivially only on those tensor factors of $\mathcal{H}_{\mathrm{L}}(\sigma)=\mathcal{K}_{\mathrm{sp}}^{\otimes \sigma_{1}}$ which correspond to the subsurface $\Sigma_{m} \hookrightarrow \Sigma$. More precisely, let $\mathbb{E}_{m} \subset \sigma_{\mathrm{I}}$ be the set of edges in $\sigma_{1}$ which have nontrivial intersection with $\Sigma_{m}$. Out of $\mathrm{U}^{\prime}(m)$ let us then define the operator $\mathrm{U}(m): \mathcal{K}\left(\sigma_{m}\right) \rightarrow \mathcal{K}\left(\tau_{m}\right)$ by applying definition (B.5) to the case $\mathrm{O} \equiv \mathrm{U}^{\prime}(m)$ and $\mathbb{J} \equiv \mathbb{E}_{m}$.

Comparison. The crucial difference between $\widetilde{U}(m)$ and $\mathrm{U}(m)$ is that the latter is manifestly acting locally in $\mathcal{H}_{\mathrm{L}}(\sigma)$, in the sense that it acts only on the tensor factor of $\mathcal{H}_{\mathrm{L}}(\sigma)$ which corresponds to the subsurface $\Sigma_{m}$. This is not obvious in the case of $\widetilde{U}(m)$.

The length operators $\mathrm{L}_{\sigma, c}$ associated to the boundary components $c \in A(\Sigma)$ form a commutative family of operators. The joint spectral decomposition for this family of operators leads us to represent $\mathcal{H}_{\mathrm{L}}(\Sigma)$ as

$$
\mathcal{H}_{\mathrm{L}}(\Sigma) \simeq \int_{\mathfrak{L}} d \eta_{\Sigma}(\mathfrak{c}) \mathcal{H}_{\mathrm{L}}(\Sigma, \mathfrak{c})
$$

where the integration is extended over the set $\mathfrak{L} \simeq \mathbb{R}_{+}^{s}$ of all colorings $\mathfrak{c}$ of the boundary by elements of $\mathbb{R}_{+}$. It follows from Theorem 6 that the operators $\mathbf{U}(m), \widetilde{U}(m)$ commute (up to permutations of the boundary components) with the length operators $\mathrm{L}_{\sigma, c}, c \in A(\Sigma)$. Within the representation (17.2) one may therefore ${ }^{10}$ represent $\mathrm{U}(m)$ and $\widetilde{\mathrm{U}}(m)$ by families of operators $(\mathrm{U}(m, \mathfrak{c}))_{\mathfrak{c} \in \mathfrak{L}}$ and $(\widetilde{\mathrm{U}}(m, \mathfrak{c}))_{\mathfrak{c} \in \mathfrak{L}}$, respectively.

\subsection{The main result}

The following theorem is the main result of this paper.

Theorem 8. The operators $\mathrm{U}(m, \mathfrak{c}), \mathfrak{c} \in \mathfrak{L}$, generate a tower of projective unitary representations of the modular groupoids $\mathrm{M}(\Sigma)$.

The proof of Theorem 8 will take up the rest of this subsection.

To begin with, let us note that the necessary structure (12.2) of the Hilbert spaces follows trivially from our definition of the length representation in Section 16, where in the present case we simply have $\mathcal{H}\left(S_{3}, \mathfrak{c}_{3}\right) \simeq \mathbb{C}$. It is furthermore clear that the operators $\widetilde{\mathrm{U}}(m, \mathfrak{c}), \mathfrak{c} \in \mathfrak{L}$ generate a unitary projective representation of the modular groupoid $\mathrm{M}(\Sigma)$ for each surface $\Sigma$ within the considered class. Let us finally note that the naturality properties formulated in Subsection 12.3 clearly hold for the operators $\mathrm{U}(m, \mathfrak{c}), \mathfrak{c} \in \mathfrak{L}$. Our main task is therefore to show that $\widetilde{\mathbf{U}}(m, \mathfrak{c})=\mathbf{U}(m, \mathfrak{c})$, as will be established in Proposition 13 below.

\footnotetext{
${ }^{10}$ According to Proposition 14 in Appendix B
} 
Proposition 13. For all $m \in \mathcal{M}_{1}^{\mathrm{ad}}(\Sigma)$ there exists a path $\pi_{m} \in\left[\theta_{m}, \varphi_{m}\right]$ such that we have

$$
\widetilde{\mathrm{U}}(m, \mathfrak{c})=\mathrm{U}(m, \mathfrak{c})
$$

Proof. When we compare the respective definitions of $\mathrm{U}(m)$ and $\widetilde{\mathrm{U}}(m)$, we observe that there are two main discrepancies that we need to deal with. First, it is not always true that the path $\pi_{m}$ can be realized locally in the sense of Definition 7. It may therefore not be clear a priori why there should exist a simple relation between $\mathrm{U}(m)$ and $\widetilde{\mathrm{U}}(m)$.

Second, we may observe that the definition of the operators $\mathrm{U}(m)$ and $\widetilde{\mathrm{U}}(m)$ involves products of operators $\mathrm{C}_{\sigma, p}$, where the set of vertices $p$ that the product is extended over is generically much smaller in the case of $\mathrm{U}(m)$. This means that most of the factors $C_{\sigma, p}$ must ultimately cancel each other in the expression for $\widetilde{\mathrm{U}}(m)$. The first step will be to prove Proposition 13 in the case that $m$ can be realized locally.

Lemma 14. We have

$$
\widetilde{\mathrm{U}}(m)=\mathrm{U}(m)
$$

whenever $m=\left[\tau_{m}, \sigma_{m}\right] \in \mathcal{M}_{\mathbf{1}}^{\prime}(\Sigma)$ can be realized locally.

Proof. It follows from (15.11) and (14.5) that $C_{\sigma, p}$ can be represented as a function of the following operators

$$
\mathrm{C}_{\sigma, p}=\mathrm{C}_{\sigma, p}\left(\mathrm{p}_{v_{p}}, \mathrm{q}_{v_{p}} ; \mathrm{L}_{c_{2}}, \mathrm{~L}_{c_{1}}\right),
$$

where $v_{p}$ is the vertex of $\varphi_{\sigma}$ contained in the trinion $T_{p}$ and $c_{\epsilon} \equiv c_{\epsilon}(p), \epsilon=1,2$ are the curves which represent the outgoing and incoming boundary components of $T_{p}$ respectively. Let us recall that the length operators $\mathrm{L}_{\tau_{m}, c_{\epsilon}}$ and $\mathrm{L}_{\sigma_{m}, c_{\epsilon}} \epsilon=1,2$ satisfy (15.6). These observations imply that

$$
\mathrm{u}\left(\pi_{m}\right) \cdot \mathrm{C}_{\sigma_{m}, p}=\mathrm{C}_{\tau_{m}, p} \cdot \mathrm{u}\left(\pi_{m}\right),
$$

whenever the operators $\mathrm{p}_{\sigma, p}$ and $\mathrm{q}_{\sigma, p}$ commute with $\mathrm{u}\left(\pi_{m}\right)$. Our task is therefore to determine the set of all $p \in \sigma_{\mathrm{o}}$ for which this is the case. The condition that $m$ can be realized locally implies that (17.4) will hold unless $p$ is located within $\Sigma_{m}$. The claim now follows straightforwardly from these observations.

In order to treat the general case let us recall that Proposition 10 implies that $\pi_{m}$ may be chosen as $\pi_{m}=y_{m} \circ \hat{\pi}_{m} \circ y_{m}^{-1}$, where $y_{m}$ is uniquely defined by the factorization of $Y_{m}$ into elementary moves that can be realized locally, and $\hat{\pi}_{m}$ is the (fixed) path which was chosen to represent $m$ in the case that $m$ can be realized locally. This leads to the following representation for $\widetilde{\mathrm{U}}(m)$ :

$$
\begin{aligned}
\widetilde{\mathrm{U}}(m) & =\mathrm{D}\left(\tau_{m}\right) \cdot \mathrm{u}\left(\pi_{m}\right) \cdot \mathrm{D}\left(\sigma_{m}\right)^{\dagger} \\
& =\mathrm{D}\left(\tau_{m}\right) \cdot \mathrm{u}\left(y_{m}\right) \cdot \mathrm{u}\left(\hat{\pi}_{m}\right) \cdot \mathrm{u}\left(y_{m}^{-1}\right) \cdot \mathrm{D}\left(\sigma_{m}\right)^{\dagger} .
\end{aligned}
$$

By using Lemma 14 one may deduce from (17.5) that the following holds:

$$
\begin{aligned}
\widetilde{\mathbf{U}}(m) & =\widetilde{\mathbf{U}}\left(Y_{m}\right) \cdot \mathbf{U}(m) \cdot \widetilde{\mathbf{U}}\left(Y_{m}^{-1}\right) \\
& =\mathbf{U}\left(Y_{m}\right) \cdot \mathbf{U}(m) \cdot \mathbf{U}\left(Y_{m}\right)^{\dagger} .
\end{aligned}
$$

It remains to observe that 
Lemma 15. We have $\mathrm{U}\left(m_{1}\right) \cdot \mathrm{U}\left(m_{2}\right) \cdot \mathrm{U}\left(m_{1}\right)^{\dagger}=\mathrm{U}\left(m_{2}\right)$ whenever $\operatorname{supp}\left(m_{1}\right) \cap \operatorname{supp}\left(m_{2}\right)=\emptyset$.

Recall that we had defined the notation $\operatorname{supp}(m)$ by $\operatorname{supp}(m)=\{p\}$ if $m=Z_{p}, B_{p}, S_{p}$ and $\operatorname{supp}(m)=\{p, q\}$ if $m=(p q), F_{p q}$.

Proof. Let us factorize $\mathcal{H}_{\mathrm{L}}(\Sigma)$ as $\mathcal{H}_{\mathrm{L}}(\Sigma)=\mathcal{K}_{\mathrm{sp}}^{\otimes \beta_{1}} \otimes \mathcal{K}_{\mathrm{sp}}^{\otimes \sigma_{1} \backslash \beta_{1}}$, where $\beta_{1} \subset \sigma_{1}$ is the set of all edges which end in boundary components of $\Sigma$. The representation 17.2 may be rewritten as

$$
\mathcal{H}_{\mathrm{L}}(\Sigma) \simeq \int_{\mathbb{R}_{+}^{s}}^{\oplus} d \eta_{s}(\mathbf{c}) \mathcal{H}_{\mathrm{L}}(\Sigma, \mathbf{c})
$$

where $\mathcal{H}_{\mathrm{L}}(\Sigma, \mathbf{c}) \simeq \mathcal{K}_{\mathrm{sp}}^{\otimes \sigma_{\perp} \backslash \beta_{1}}$ for all $\mathbf{c} \in \mathbb{R}_{+}^{s}$. Let $\mathrm{U}(m, \mathbf{c}), \mathbf{c} \in \mathbb{R}_{+}^{s(m)}$ be the unitary operators on $\mathcal{H}_{\mathrm{L}}\left(\Sigma_{m}, \mathbf{c}\right)$ which represent the operators $\mathrm{U}(m)$ in the representation (17.7). Within this representation it becomes almost trivial to complete the proof of Proposition 13. Let $\beta_{21}=$ $\beta_{2} \cup \beta_{1}$, where $\beta_{\jmath} \subset \sigma_{1}, \jmath=1,2$ are the sets of edges which correspond to boundary components of $\Sigma_{m_{\jmath}}$. Let $\delta_{\jmath} \subset \sigma_{1}, \jmath=1,2$ be the sets of edges that are fully contained in the interior of $\Sigma_{m_{\jmath}}$ respectively. Let finally $\sigma_{1}^{\prime}=\sigma_{1} \backslash\left(\beta_{21} \cup \delta_{2} \cup \delta_{1}\right)$ We may then factorize $\mathcal{H}_{\mathrm{L}}(\Sigma)$ in the following way:

$$
\mathcal{H}_{\mathrm{L}}(\Sigma)=\mathcal{K}_{\mathrm{sp}}^{\otimes \beta_{21}} \otimes \mathcal{K}_{\mathrm{sp}}^{\otimes \delta_{2}} \otimes \mathcal{K}_{\mathrm{sp}}^{\otimes \delta_{1}} \otimes \mathcal{K}_{\mathrm{sp}}^{\otimes \sigma_{1}^{\prime}}
$$

where we define $\mathcal{K}_{\mathrm{sp}}^{\otimes \delta}=\mathbb{C}$ if $\delta=\emptyset$. This may be rewritten as

$$
\mathcal{H}_{\mathrm{L}}(\Sigma) \simeq \int_{\mathbb{R}_{+}^{s_{21}}} d \mu\left(\mathbf{c}_{21}\right) \mathcal{H}_{\mathrm{L}}\left(\Sigma, \mathbf{c}_{21}\right)
$$

where $\mathcal{H}_{\mathrm{L}}\left(\Sigma, \mathbf{c}_{21}\right) \simeq \mathcal{K}_{\mathrm{sp}}^{\otimes \delta_{2}} \otimes \mathcal{K}_{\mathrm{sp}}^{\otimes \delta_{1}} \otimes \mathcal{K}_{\mathrm{sp}}^{\otimes \sigma_{1}^{\prime}}$ for all $\mathbf{c}_{21} \in \mathbb{R}_{+}^{s_{21}}, s_{21}=\operatorname{card}\left(\beta_{21}\right)$. In the representation (17.9) we may represent $\mathrm{U}\left(m_{\jmath}\right), \jmath=1,2$ by families of operators $\mathrm{U}\left(m_{\jmath}, \mathbf{c}_{21}\right)$ which take the form $\mathbf{U}\left(m_{2}, \mathbf{c}_{21}\right) \simeq \mathbf{U}_{2}\left(m_{2}, \mathbf{c}_{21}\right) \otimes \mathrm{id} \otimes \mathrm{id}$ and $\mathbf{U}\left(m_{1}, \mathbf{c}_{21}\right) \simeq \mathrm{id} \otimes \mathbf{U}_{1}\left(m_{1}, \mathbf{c}_{21}\right) \otimes \mathrm{id}$ respectively. The Lemma follows easily from these observations.

It follows from the Lemma that $\mathrm{U}\left(Y_{m}\right) \cdot \mathrm{U}(m) \cdot \mathrm{U}\left(Y_{m}\right)^{\dagger}=\mathrm{U}(m)$, which completes the proof of Proposition 13.

Taken together our results show that the quantization of Teichm üler spaces yields a tower of unitary projective representations of the modular groupoid in the sense of $\S 12.3$.

\subsection{Concluding remarks}

Let us recall that the mapping class group is a group of symmetries for both complexes $\mathcal{P} t(\Sigma)$ and $\mathcal{M}(\Sigma)$. Having projective unitary representations of the associated groupoids $\operatorname{Pt}(\Sigma)$ and $\mathrm{MC}(\Sigma)$ will therefore induce corresponding representations of the mapping class group by means of the construction in $\S 11.2$. It is not hard to see It follows quite easily from Proposition 7 that these two representations are equivalent.

Finally it is clearly of interest to calculate the phases in the relations $u\left(\pi_{\varpi}\right)=\zeta^{\nu(\varpi)}$.

Conjecture 1. There exists a definition for the operators $\mathrm{U}(m), m \in \mathcal{M}_{1}(\Sigma)$ such that the phases $\zeta^{\hat{\nu}(\varpi)}$ which appear in the relations $\mathrm{u}\left(\pi_{\varpi}\right)=\zeta^{\nu(\varpi)}$ are trivial for all but one $\varpi \in$ 
$\mathcal{M}_{2}(\Sigma)$, which can be chosen as the relation (13.9),a). The phase which appears in the relation (13.9), a) is given as

$$
\zeta^{\nu(\varpi)}=e^{\frac{\pi i}{2} c_{\mathrm{L}}}, \quad c_{\mathrm{L}} \equiv 1+6\left(b+b^{-1}\right)^{2} .
$$

We now believe to have a proof of this conjecture. Details will appear elsewhere. Our conjecture is also strongly supported by the calculation in [Ka2] which establishes a similar result for the realization of the pure mapping class group on the quantized Teichm uller spaces. 


\section{A. The special functions $e_{b}(x)$ and $s_{b}(x)$}

The function $s_{b}(x)$ may be defined with the help of the following integral representation.

$$
\log s_{b}(x)=\frac{1}{i} \int_{0}^{\infty} \frac{d t}{t}\left(\frac{\sin 2 x t}{2 \sinh b t \sinh b^{-1} t}-\frac{x}{t}\right) .
$$

This function, or close relatives of it like

$$
e_{b}(x)=e^{\frac{\pi i}{2} x^{2}} e^{-\frac{\pi i}{24}\left(2-Q^{2}\right)} s_{b}(x),
$$

have appeared in the literature under various names like "Quantum Dilogarithm" [FK], "Hyperbolic G-function" [Ru], "Quantum Exponential Function" [Wor] and "Double Sine Function", we refer to the appendix of [KLS] for a useful collection of properties of $s_{b}(x)$ and further references. The most important properties for our purposes are

(i) Functional equation: $s_{b}\left(x-i \frac{b}{2}\right)=2 \cosh \pi b x s_{b}\left(x+i \frac{b}{2}\right)$.

(ii) Analyticity: $s_{b}(x)$ is meromorphic,

$$
\begin{aligned}
& \text { poles: } x=c_{b}+i\left(n b-m b^{-1}\right), n, m \in \mathbb{Z}^{\geq 0} . \\
& \text { zeros: } x=-c_{b}-i\left(n b-m b^{-1}\right), n, m \in \mathbb{Z}^{\geq 0} .
\end{aligned}
$$

(iii) Self-duality: $\quad s_{b}(x)=s_{1 / b}(x)$.

(iv) Inversion relation: $s_{b}(x) s_{b}(-x)=1$.

(v) Unitarity: $\overline{s_{b}(x)}=1 / s_{b}(\bar{x})$.

(vi) Residue: $\operatorname{res}_{x=c_{b}} s_{b}(x)=e^{-\frac{\pi i}{12}\left(1-4 c_{b}^{2}\right)}(2 \pi i)^{-1}$.

The function $e_{b}(x)$ clearly has very similar properties as $s_{b}(x)$. We list the properties that get modified compared to $s_{b}(x)$ below.

(i)' Functional equation: $e_{b}\left(x-i \frac{b}{2}\right)=\left(1+e^{2 \pi b x}\right) e_{b}\left(x+i \frac{b}{2}\right)$.

(iv)' Inversion relation: $e_{b}(x) e_{b}(-x)=e^{\pi i x^{2}} e^{-\frac{\pi i}{6}\left(1+2 c_{b}^{2}\right)}$.

(vi)' Residue: $\operatorname{res}_{x=c_{b}} s_{b}(x)=(2 \pi i)^{-1}$.

Among the most remarkable properties satisfied by the function $e_{b}(x)$ is the so-called pentagonal relation (A.12) which underlies the validity of the pentagonal relation (11.34),

$$
e_{b}(\mathbf{p}) \cdot e_{b}(\mathbf{q})=e_{b}(\mathbf{q}) \cdot e_{b}(\mathbf{p}+\mathbf{q}) \cdot e_{b}(\mathbf{p})
$$

Relation (A.12) is valid if $\mathrm{q}$ and p represent $[\mathrm{p}, \mathrm{q}]=(2 \pi i)^{-1}$ on $L^{2}(\mathbb{R})$. Equation (A.12) in turn is equivalent to the following property of the function $E_{b}(x) \equiv e_{b}\left(-\frac{x}{2 \pi b}\right)$ :

$$
E_{b}(\mathrm{U}) \cdot E_{b}(\mathrm{~V})=E_{b}(\mathrm{U}+\mathrm{V})
$$

where $\mathrm{U}=e^{2 \pi b \mathrm{q}}, \mathrm{V}=e^{2 \pi b \mathrm{p}}$. Proofs of (A.12) and (A.13) can be found in [Wor, FKV, BT, Vo]. 


\section{B. Operator-theoretical background}

Let $\mathcal{H}$ be a separable Hilbert space. The algebra of bounded operators in $\mathcal{H}$ will be denoted by $\mathbf{B}(\mathcal{H})$. We will only need some of the most basic notions and results from functional analysis and the theory of operator algebras as summarized e.g. in [Wa, Chapter 14]. For the reader's convenience and to fix some notations we shall formulate the results that we need below.

Theorem 9. - [Wa, Theorem 14.8.14] -

Let $\mathbf{C}$ be a commutative von Neumann subalgebra of $\mathbf{B}(\mathcal{H})$, where $\mathcal{H}$ is a separable Hilbert space. Then there exists a compact, separable Hausdorff space $X$, a Radon measure $\mu$ on $X, a$ measurable family of Hilbert spaces $\left\{\mathcal{H}_{x}\right\}_{x \in X}$, and a unitary bijection

$$
\mathrm{U}: \mathcal{H} \rightarrow \mathcal{H}_{\mathbf{C}} \equiv \int_{X} d \mu(s) \mathcal{H}_{s}
$$

such that

$$
\mathrm{U} \cdot \mathbf{C} \cdot \mathbf{U}^{\dagger}=\left\{\mathrm{m}_{f} ; f \in L^{\infty}(x, d \mu)\right\}
$$

where $\mathrm{m}_{f}$ is the multiplication operator defined by

$$
\mathrm{m}_{f}: \mathcal{H}_{\mathbf{C}} \ni\left\{\Psi_{x}\right\}_{x \in X} \rightarrow\left\{f(x) \Psi_{x}\right\}_{x \in X} \in \mathcal{H}_{\mathbf{C}}
$$

If $\mathbf{C}$ is a commutative von Neumann subalgebra of $\mathbf{B}(\mathcal{H})$ we will call an operator $\mathrm{O}$ on $\mathcal{H}$ C-decomposable if there exists a family of bounded operators $\mathrm{O}_{x}$, defined on $\mathcal{H}_{x}$ for $\mu$-almost every $x \in X$, such that

$$
\mathrm{U} \cdot \mathrm{O} \cdot \mathrm{U}^{\dagger}=\int_{X} d \mu(s) \mathrm{O}_{x}
$$

Let $\mathbf{D}_{\mathbf{C}}$ be the algebra of all $\mathbf{C}$-decomposable operators.

Proposition 14. - [Wa, Proposition 14.8.8] - $\mathbf{D}_{\mathbf{C}}$ is the commutant of $\mathbf{C}$ within $\mathbf{B}(\mathcal{H})$.

The unbounded operators that we will have to deal with will all be self-adjoint. We will freely use standard functional calculus for self-adjoint operators. When we say that two unbounded self-adjoint operators commute,

$$
[\mathrm{A}, \mathrm{B}]=0 \text {, }
$$

we will always mean commutativity of the spectral projections. Let $\mathcal{F}=\left\{\mathrm{A}_{\imath} ; \imath \in \mathcal{I}\right\}$ be a family of commuting self-adjoint operators defined on dense domains in a separable Hilbert space $\mathcal{H}$. Standard functional calculus for commuting self-adjoint operators associates to $\mathcal{F}$ a commutative von Neumann subalgebra $\mathbf{C}_{\mathcal{F}}$ of $\mathbf{B}(\mathcal{H})$. Theorem 9 applied to $\mathbf{C}_{\mathcal{F}}$ yields the existence of a common spectral decomposition for the family $\mathcal{F}$, where $X$ represents the onepoint compactification of the spectrum of $\mathcal{F}$.

\section{Tensor product notation}

For a given finite set $I$ we will often use the notation $\mathcal{H}^{\otimes \mathbb{I}}$ instead of $\mathcal{H}^{\otimes \operatorname{card}(\mathbb{I})}$, where card(II) is the number of elements in $\mathbb{I}$. In order to avoid fixing a numbering of the elements of $\mathbb{I}$ we will 
find it useful to employ the following "leg-numbering" notation. To a given a subset $\mathbb{I} \subset \mathbb{I}$ we may associate the canonical permutation operators

$$
\mathrm{P}_{\mathbb{J}}: \mathcal{H}^{\otimes \mathbb{I}} \rightarrow \mathcal{H}^{\otimes \mathbb{I}} \otimes \mathcal{H}^{\otimes \mathbb{I} \backslash \mathbb{I}}
$$

To an operator $\mathrm{O} \in \mathbf{B}\left(\mathcal{H}^{\otimes \mathbb{I}}\right)$ we may then associate an operator $\mathrm{O}_{\mathbb{J}} \in \mathbf{B}\left(\mathcal{H}^{\otimes I}\right)$ via

$$
\mathrm{O}_{\mathbb{J}} \equiv \mathrm{P}_{\mathbb{J}}^{-1} \cdot(\mathrm{O} \otimes \mathrm{id}) \cdot \mathrm{P}_{\mathbb{J}}
$$

If $\mathbb{J}=\left\{i_{1}, i_{2}, \ldots\right\}$ we will sometimes write $\mathrm{O}_{i_{1} i_{2} \ldots}$ instead of $\mathrm{O}_{\mathbb{J}}$. We will also abbreviate $\mathrm{O}_{\mathbb{J}_{1} \cup \mathbb{J}_{2} \cup \ldots}$ to $\mathrm{O}_{\mathbb{J}_{1} \mathbb{J}_{2} \ldots}$.

\section{On the proof of Theorem 2}

A similar statement is known [CF1] for the closely related groupoid $\widetilde{\mathrm{Pt}}(\Sigma)$ whose elements are the moves $\left[\tilde{\varphi}_{2}, \tilde{\varphi}_{1}\right]$ between fat graphs $\tilde{\varphi}_{2}, \tilde{\varphi}_{1}$ which do not have the decoration introduced in Subsection 5.1, but which have a numbering of the edges instead.

Theorem 2'. The groupoid $\widetilde{\mathrm{Pt}}(\Sigma)$ is the path groupoid of the complex $\widetilde{\mathcal{P} t}(\Sigma)$ which has vertices $\tilde{\varphi}$ and the following generators and relations:

\section{Generators:}

(i) Flips $F_{e}$ along the edges $e \in \tilde{\varphi}_{1}$ (see Figure (7)).

(ii) The exchanges (ef) of the numbers assigned to edges $e$ and $f$.

\section{Relations:}

(a) There is no vertex that both edges e and $f$ are incident to: $F_{f} \circ F_{e}=F_{e} \circ F_{f}$.

(b) The edges $e$ and $f$ are incident to the same vertex: $F_{e} \circ F_{f} \circ F_{e} \circ F_{f} \circ F_{e}=(e f)$.

(c) $(e f) \circ F_{e}=F_{f} \circ(e f)$

(d) $F_{e} \circ F_{e}=$ id.

It was observed in $[\mathrm{P} 3, \mathrm{CF} 1, \mathrm{CP}]$ that Theorem 2' follows quite easily from the fact $[\mathrm{P} 1, \mathrm{Ko}]$ that the fat graphs $\tilde{\varphi}$ can be used to label cells in certain cell decompositions $[\mathrm{Ha}, \mathrm{P} 1]$ of $\mathcal{T}(\Sigma) \times \mathbb{R}_{+}^{s}$. This allows one to associate a path $\hat{\pi}$ in $\mathcal{T}(\Sigma) \times \mathbb{R}_{+}^{s}$ to each path $\tilde{\pi} \in\left[\tilde{\varphi}_{2}, \tilde{\varphi}_{1}\right]$ in $\widetilde{\operatorname{Pt}}(\Sigma)$. Each closed path $\hat{\pi}$ in $\mathcal{T}(\Sigma) \times \mathbb{R}_{+}^{s}$ can be deformed into small circles around the codimension two faces of the cell decompositions from [Ha, P1]. The latter are easily identified with the relations listed in Theorem 2'. This implies that no relations other than those listed in Theorem 2' are needed to contract a closed path $\tilde{\pi} \in[\tilde{\varphi}, \tilde{\varphi}]$ to the identity.

It remains to show that Theorem 2 follows from Theorem 2'. To this aim it is important to observe that $\widetilde{\operatorname{Pt}}(\Sigma)$ imbeds into $\operatorname{Pt}(\Sigma)$ by means of the following construction: Given the numbered fat graph $\tilde{\varphi}$ let us define a decorated fat graph $\varphi$ according to the following rule: Pick any numbering of the vertices of $\tilde{\varphi}$. For each vertex $v$ let us distinguish among the edges which emanate from $v$ the one with the smallest number assigned to it. For each move $F_{e}$ let us choose a lift $\phi_{e}$ of the form $\phi_{e}=\delta_{e}^{\prime} \circ \omega_{u_{e} v_{e}} \circ \delta_{e}$, where the vertices $u_{e}$ and $v_{e}$ represent are the ends of $e$ and $\delta_{e}^{\prime}, \delta_{e}$ change the decoration only. It is then crucial to check that the relations of $\operatorname{Pt}(\Sigma)$ ensure that the image of $\widetilde{\operatorname{Pt}}(\Sigma)$ within $\operatorname{Pt}(\Sigma)$ is simply connected. This follows if the images of the relations listed in Theorem 2' are contractible within $\operatorname{Pt}(\Sigma)$. We have drawn a 
particular example for such an image in Figure 24 below. It is easy to verify that the image of the corresponding path in $\operatorname{Pt}(\Sigma)$ is closed. More generally one needs to consider all relations obtained by changes of the numbering of the edges. These relations are obtained by inserting $\eta^{-1} \circ \eta$ between the moves which occur in the image of the relation under consideration, where $\eta$ represents the change of decoration induced by a change of numbering. In a similar way one may convince oneself that all the relations listed in Theorem 2' are mapped to closed paths in $\operatorname{Pt}(\Sigma)$, which completes the proof that $\widetilde{\operatorname{Pt}}(\Sigma)$ embeds into $\operatorname{Pt}(\Sigma)$.

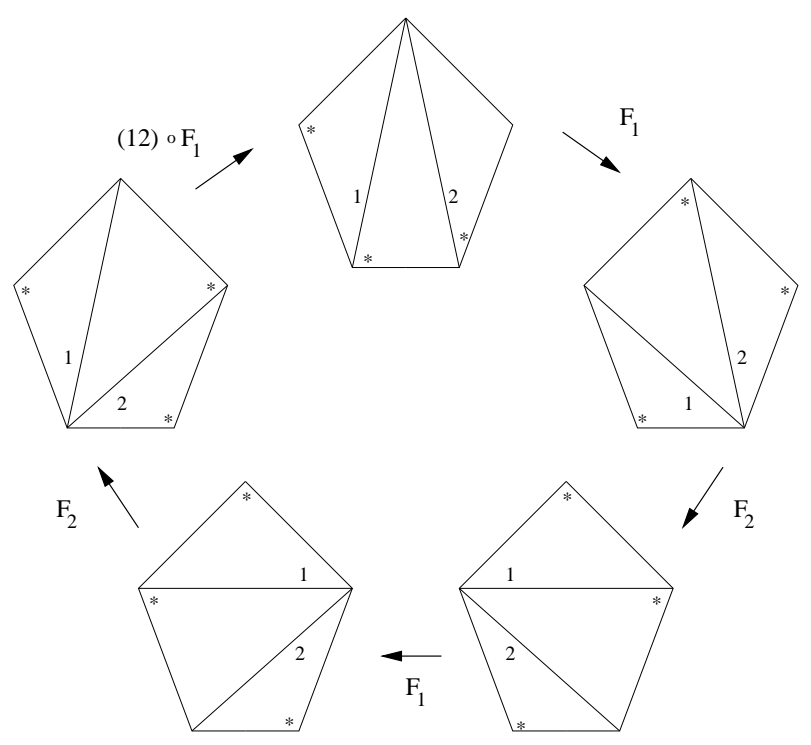

Figure 24: Image of the pentagon in $\operatorname{Pt}(\Sigma)$.

It remains to show that each closed path in $\operatorname{Pt}(\Sigma)$ is homotopic to a closed path in the image of $\widetilde{\operatorname{Pt}}(\Sigma)$. Let us consider a closed path $\pi \in[\varphi, \varphi] \in \operatorname{Pt}(\Sigma)$. Write $\pi=\omega_{n} \circ \cdots \circ \omega_{1}$, where each $\omega_{k}$ is of the form $\omega_{k}=\delta_{k}^{\prime} \circ \omega_{u_{k} v_{k}} \circ \delta_{k}$, with $\delta_{k}, \delta_{k}^{\prime}$ being composed out of moves $\rho_{w}$ and permutations only. Choose any numbering for the edges of $\varphi$ and denote the resulting numbered fat graph by $\tilde{\varphi}$. The path $\pi$ then defines a path $\tilde{\pi}$ in $\widetilde{\operatorname{Pt}}(\Sigma)$ by means of the following construction: Substitute the moves $\omega_{r}$ for $k=1, \ldots, n$ by the corresponding flips along the edges which connect $u_{k}$ and $v_{k}$, and then multiply the result by the necessary permutations of the numbers assigned to the edges of $\tilde{\varphi}$. $\tilde{\pi}$ has the form $\tilde{\pi}=\Phi_{n} \circ \cdots \circ \Phi_{1}$, where $\Phi_{k} \in\left[\tilde{\varphi}_{k+1}, \tilde{\varphi}_{k}\right]$ factorize into a single flip $F_{k}$ times a change of numbering $P_{k}, \Phi_{k}=P_{k} \circ F_{k}$.

The path $\tilde{\pi}$ is mapped to a path $\pi^{\prime}$ in $\operatorname{Pt}(\Sigma)$ by means of the construction above. $\pi^{\prime}$ has the form $\pi^{\prime}=\phi_{n}^{\prime} \circ \cdots \circ \phi_{1}^{\prime}$ with $\phi_{k}^{\prime} \in\left[\varphi_{k+1}, \varphi_{k}\right]$. On the other hand let us note that the path $\pi^{\prime \prime}=\phi_{n}^{\prime \prime} \circ \cdots \circ \phi_{1}^{\prime \prime}$ with $\phi_{k}^{\prime \prime} \equiv \eta_{k+1}^{-1} \circ \omega_{k} \circ \eta_{k}$ is clearly homotopic to $\pi$ for all changes of decorations $\eta_{k}$ which satisfy $\eta_{n+1}=\eta_{1}$. For suitable choice of the $\eta_{k}$ one gets $\phi_{k}^{\prime \prime} \in\left[\varphi_{k+1}, \varphi_{k}\right]$. It is then easy to see that $\phi_{k}^{\prime}$ and $\phi_{k}^{\prime \prime}$ are homotopic.

\section{Proof of Proposition 10}

One may easily convince oneself that the moves $Z_{p}$ and $B_{p}$ can generically not be realized locally. There are simple sufficient criteria for a move $m$ to be realizable locally. 


\section{Lemma 16.}

(i) A move $m=\left[\tau_{m}, \sigma_{m}\right] \in \mathcal{M}_{1}^{\prime}(\Sigma)$ can always be realized locally if all but one of the boundary components of $\Sigma_{m}$ are contained in $\left\{c_{e} ; e \in \mathcal{A}_{\sigma}\right\}$.

(ii) The moves $B_{p}, B_{p}^{\prime}, T_{p}$ can be realized locally if the curve $c_{p, 1}$ which represents the boundary component of $\mathfrak{t}_{p}$ assigned number 1 in Figure 12 is contained in $\mathcal{A}_{\sigma}$.

(iii) The moves $F_{p q}, A_{p q}, S_{p}, S_{p q}$ and $B_{p q}$ can always be realized locally.

Proof. Straightforward verifications.

Proposition 15. ( $\equiv$ Proposition 10) If $m \in \mathcal{M}_{1}^{\prime}(\Sigma)$ is admissible, but can not be realized locally, there always exists a path $\varpi_{m}$ which is (i) homotopic to $m$ within $\mathcal{M}_{1}^{\mathrm{ad}}(\Sigma)$, and (ii) takes the form

$$
\varpi_{m}=Y_{m} \circ m \circ Y_{m}^{-1}
$$

where $Y_{m}$ is a chain composed out of $Z_{p}$-moves and $F_{p q}$-moves which can all be realized locally.

Proof. The claim will follow easily from another auxiliary result that we will formulate as a separate lemma. As a preparation, let us recall that cutting a surface $\Sigma$ along the curves $c(\sigma, e)$, $e \in \mathcal{A}_{\sigma}$ produces a surface $\Sigma^{\dagger}$, the connected components of which all have genus zero. Let us call a marking $\sigma$ irreducible if the set of connected components of $\Sigma^{\dagger}$ has only one element. It is easy to see that a marking $\sigma$ is irreducible iff it has precisely one outgoing external edge.

Lemma 17. Let $\sigma$ be a marking on a surface $\Sigma$ that is irreducible. For any chosen boundary component $b$ of $\Sigma$ there exists a chain of $Z_{p}$-moves and $F_{p q}$-moves that (i) preserves admissibility in each step, (ii) consists only of moves that can be realized locally, and (iii) transforms $\sigma$ to a irreducible marking $\sigma^{\prime}$ whose outgoing external edge ends in the chosen component $b$.

Sketch of proof. In order to check the following arguments it may be useful to think of an irreducible marking as being obtained from the corresponding marking $\sigma^{\dagger}$ on the surface $\Sigma^{\dagger}$ of genus zero by identifying the appropriate boundary circles. To each incoming boundary component of $\Sigma^{\dagger}$ there corresponds a unique element of $\mathcal{A}_{\sigma}$.

The following claim is easy to verify. By means of $F_{p q}$-moves one may transform $\sigma$ to a marking $\tilde{\sigma}$ which has the following two properties. First, for each vertex $p \in \tilde{\sigma}_{\mathrm{o}}$ at least one of the edges that are incident at $p$ is contained in $\mathcal{A}_{\sigma}$. Second, there is no edge $e \in \sigma_{\mathrm{I}}$ which connects a vertex $p$ to itself. These two properties insure that for each $p \in \tilde{\sigma}_{\mathrm{o}}$ either $Z_{p}$ or $Z_{p}^{-1}$ preserves the admissibility of the marking. It is then not very hard to construct a sequence of $Z_{p}$-moves that (i) can all be realized locally and (ii) which transform $\tilde{\sigma}$ to a marking $\tilde{\sigma}^{\prime}$ whose outgoing external edge ends in the chosen boundary component $b$.

By means of a chain of $F_{p q}$-moves one may finally transform $\tilde{\sigma}^{\prime}$ back to a marking $\sigma^{\prime}$ that differs from the original marking only by the desired change of decoration.

End of proof of Lemma 15. Lemma 16 allows us to restrict attention to the cases $m=$ $Z_{p}, B_{p}, B_{p}^{\prime}, T_{p}$. We need to transform the original marking to another one which has the property that both incoming boundary components of the trinion $\mathfrak{t}_{p}$ are contained in $\mathcal{A}_{\sigma}$. Given a curve $c \in \mathcal{C}_{\sigma} \backslash \mathcal{A}_{\sigma}$ there is a unique subsurface $\Sigma_{c} \hookrightarrow \Sigma$ with marking $\sigma_{c}$ such that (i) $c$ is the unique 


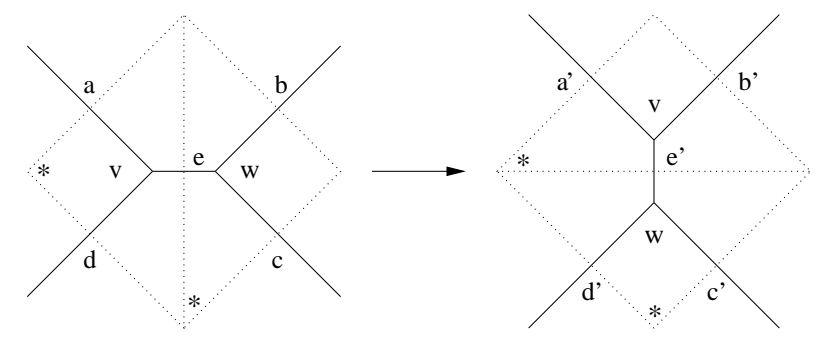

Figure 25: Labelling of the edges which are relevant for the description of a flip.

outgoing curve in the boundary of $\Sigma_{c}$, and (ii) the incoming boundary curves of $\Sigma_{c}$ are contained in $\mathcal{A}_{\sigma}$. In order to infer the existence of the chain $Y_{p}$ it clearly suffices to apply Lemma 17 to the subsurfaces $\Sigma_{c_{1}}$ and $\Sigma_{c_{2}}$ respectively, where $c_{1}$ and $c_{2}$ are the incoming boundary components of the trinion with label $p$.

Lemma 15 implies that $\pi_{m}$ may be chosen as $\pi_{m}=y_{m} \circ \hat{\pi}_{m} \circ y_{m}^{-1}$, where $y_{m}$ is uniquely defined by the factorization of $Y_{m}$ into elementary moves that can be realized locally, and $\hat{\pi}_{m}$ is the (fixed) path which was chosen to represent $m$ in the case that $m$ can be realized locally.

\section{E. On the proof of Theorem 6}

\section{E.1 A technical preliminary}

To begin with, let us present a technical result that facilitates the explicit computations. The operator functions we will be interested in are of the form

$$
\mathrm{O} \equiv \mathrm{O}\left(\left\{\mathrm{z}_{\imath} ; \imath \in \varphi_{\mathbf{1}}\right\}\right)=\sum_{\tau \in \mathcal{F}} C_{\tau} e^{\mathrm{x}(\tau)}, \quad \mathrm{x}(\tau) \equiv \sum_{e \in \varphi_{\mathbf{l}}} \tau(e) \mathrm{z}_{e}
$$

where the summation is taken over the space $\mathcal{F}$ of all maps $\varphi_{1} \ni e \rightarrow \tau(e) \in \frac{1}{2} \mathbb{Z}$, and the coefficients $C_{\tau}$ are assumed to be non-vanishing for a finite number of $\tau \in \mathcal{F}$ only, in which case we assume $C_{\tau} \in \mathbb{R}_{+}$. These operators are densely defined and positive self-adjoint due to the self-adjointness of $x(\tau)$. The cone generated by operator functions of the form (E.7) will be denoted $\mathbf{C}_{+}(\varphi)$.

Proposition 16. Let $\varphi, \varphi^{\prime}$ be two fat graphs that are related by $\rho \equiv \omega_{v w} \in \mathcal{P} t(\Sigma)$, and let us adopt the labelling of the relevant edges given by Figure 25 . For each $\mathrm{O} \in \mathrm{C}_{+}(\varphi)$ one has

$$
\mathrm{a}_{\rho}(\mathrm{O}) \equiv \mathrm{u}(\pi) \cdot \mathrm{O} \cdot \mathrm{u}(\pi)^{-1}=\mathrm{E}_{b}\left(\mathrm{z}_{e^{\prime}}\right) \cdot \mathrm{O}^{\prime} \cdot\left(\mathrm{E}_{b}\left(\mathrm{z}_{e^{\prime}}\right)\right)^{-1}
$$

where $\mathrm{E}_{b}(\mathrm{z}) \equiv e_{b}\left(-\frac{\mathrm{z}}{2 \pi b}\right)$, and $\mathrm{O}^{\prime}$ is related to $\mathrm{O}$ via

$$
\begin{aligned}
\mathrm{O}^{\prime} & \equiv \mathrm{O}\left(\mathrm{z}_{a^{\prime}}, \mathrm{z}_{b^{\prime}}+\mathrm{z}_{e^{\prime}}, \mathrm{z}_{c^{\prime}}, \mathrm{z}_{d^{\prime}}+\mathrm{z}_{e^{\prime}},-\mathrm{z}_{e^{\prime}},\left\{\mathrm{z}_{i} ; \imath \in \varphi_{\mathbf{I}} \backslash\left\{a^{\prime}, b^{\prime}, c^{\prime}, d^{\prime}, e^{\prime}\right\}\right\}\right) \text { if } \\
\mathrm{O} & =\mathrm{O}\left(\mathrm{z}_{a}, \mathrm{z}_{b}, \mathrm{z}_{c}, \mathrm{z}_{d}, \mathrm{z}_{e},\left\{\mathrm{z}_{\imath} ; \imath \in \varphi_{\mathbf{I}} \backslash\{a, b, c, d, e\}\right\}\right) .
\end{aligned}
$$

Proof. Given the explicit expression for $\mathrm{T}_{v w}$ we only have to verify that

$$
e^{-2 \pi i \mathbf{p}_{v} \mathbf{q}_{w}} \cdot \mathrm{O} \cdot e^{-2 \pi i \mathbf{p}_{v} \mathbf{q}_{w}}=\mathbf{O}^{\prime}
$$


with $\mathrm{O}$ and $\mathrm{O}^{\prime}$ being related by (E.9). Keeping in mind (7.6) we notice that the operators $\mathrm{z}_{f}$ may be represented in the form $\mathrm{z}_{f}=\mathrm{z}_{f, v}+\mathrm{z}_{f, w}$, where $v, w \in \varphi_{\mathrm{o}}$ are the vertices connected by the edge $f \in \varphi_{\mathrm{o}}$. Let us label the vertices in $\varphi_{\mathrm{o}}$ and $\varphi_{\mathrm{o}}^{\prime}$ such that the edges $a$ and $d$ are incident to the vertices $v_{a}$ and $v_{d}$ besides to the vertex $v$ respectively, and similarly for the vertices $v_{b}, v_{c}$ and $w$. This leads to the following representations for the relevant Fock variables:

$$
\begin{aligned}
& \mathrm{z}_{e}=2 \pi b\left(\mathrm{q}_{v}-\mathrm{p}_{v}+\mathrm{p}_{w}\right), \quad \mathrm{z}_{e^{\prime}}=2 \pi b\left(\mathrm{q}_{w}^{\prime}-\mathrm{q}_{v}^{\prime}-\mathrm{p}_{w}^{\prime}\right), \\
& \mathrm{z}_{a}=2 \pi b \mathrm{p}_{v}+\mathrm{z}_{a, v_{a}}, \quad \mathrm{z}_{a^{\prime}}=2 \pi b \mathbf{p}_{v}^{\prime}+\mathrm{z}_{a^{\prime}, v_{a}}, \\
& \mathrm{z}_{b}=2 \pi b\left(\mathbf{q}_{w}-\mathrm{p}_{w}\right)+\mathrm{z}_{b, v_{b}}, \quad \mathrm{z}_{b^{\prime}}=2 \pi b\left(\mathbf{q}_{v}^{\prime}-\mathrm{p}_{v}^{\prime}\right)+\mathrm{z}_{b^{\prime}, v_{b}}, \\
& \mathrm{z}_{c}=2 \pi b\left(-\mathrm{q}_{w}\right)+\mathrm{z}_{c, v_{c}}, \quad \mathrm{z}_{c^{\prime}}=2 \pi b\left(-\mathrm{q}_{w}^{\prime}\right)+\mathrm{z}_{c^{\prime}, v_{c}} \text {, } \\
& \mathrm{z}_{d}=2 \pi b\left(-\mathrm{q}_{v}\right)+\mathrm{z}_{d, v_{d}}, \quad \mathrm{z}_{d^{\prime}}=2 \pi b \mathrm{p}_{w}^{\prime}+\mathrm{z}_{d^{\prime}, v_{d}} \text {, }
\end{aligned}
$$

To complete the proof of Proposition 16 is now the matter of a straightforward calculation.

Remark 10. This yields in particular the formulae

$$
\begin{array}{rlrl}
e^{-\mathbf{z}_{a^{\prime}}}=e^{-\frac{1}{2} \mathbf{z}_{a}}\left(1+e^{-\mathbf{z}_{e}}\right) e^{-\frac{1}{2} \mathbf{z}_{a}}, & e^{-\mathbf{z}_{b^{\prime}}}=e^{+\frac{1}{2} \mathbf{z}_{b}}\left(1+e^{+\mathbf{z}_{e}}\right) e^{+\frac{1}{2} \mathbf{z}_{b}}, \\
e^{+\mathbf{z}_{d^{\prime}}}=e^{+\frac{1}{2} \mathbf{z}_{d}}\left(1+e^{+\mathbf{z}_{e}}\right) e^{+\frac{1}{2} \mathbf{z}_{a}}, & \mathbf{z}_{e^{\prime}}=-\mathbf{z}_{e}, & e^{+\mathbf{z}_{c^{\prime}}}=e^{-\frac{1}{2} \mathbf{z}_{c}}\left(1+e^{-\mathbf{z}_{e}}\right) e^{-\frac{1}{2} \mathbf{z}_{c}} .
\end{array}
$$

It is quite obvious that these transformations reduce to their classical counterparts (6.3) in the limit $b \rightarrow 0$.

As an example let us consider the monomials $\mathrm{M}_{n_{a} n_{c}}^{n_{b} n_{d}} \equiv e^{\frac{1}{2}\left(n_{a} z_{a}+n_{b} z_{b}+n_{c} z_{c}+n_{d} z_{d}\right)}$, where $n_{b}$, $b \in\{a, b, c, d\}$ are restricted by the requirement that $2 N \equiv n_{a}+n_{c}-n_{b}-n_{d}$ must be even. One then finds certain simplifications on the right hand side of (E.8):

$$
\begin{aligned}
\mathrm{E}_{b}\left(\mathrm{z}_{e}^{\prime}\right) \cdot \mathrm{M}_{n_{a} n_{c}}^{n_{b} n_{d}} \cdot\left(\mathrm{E}_{b}\left(\mathrm{z}_{e}^{\prime}\right)\right)^{-1} & =\sqrt{\mathrm{M}_{n_{a} n_{c}}^{n_{b} n_{d}}} \frac{\mathrm{E}_{b}\left(\mathrm{z}_{e}^{\prime}+\pi i b^{2} N\right)}{\mathrm{E}_{b}\left(\mathrm{z}_{e}^{\prime}-\pi i b^{2} N\right)} \sqrt{\mathrm{M}_{n_{a} n_{c}}^{n_{b} n_{d}}} \\
& =\sqrt{\mathrm{M}_{n_{a} n_{c}}^{n_{b} n_{d}}}\left[\prod_{m=-\frac{N-1}{2}}^{\frac{N-1}{2}}\left(1+q^{2 m} e^{-\mathrm{z}_{e}^{\prime}}\right)\right] \sqrt{\mathrm{M}_{n_{a} n_{c}}^{n_{b} n_{d}}} .
\end{aligned}
$$

\section{E.2 Invariance of length operators}

In the main text we have explained how to reduce the proof of Theorem 6 to the following Lemma:

Lemma 18. Assume that $m \in \mathcal{M}_{1}^{\prime}(\Sigma)$ can be realized locally. We then have the relation

$$
\mathrm{u}\left(\pi_{m}\right) \cdot \mathrm{L}_{\sigma_{m}, c}=\mathrm{L}_{\tau_{m}, c} \cdot \mathrm{u}\left(\pi_{m}\right)
$$

for all curves $c$ such that $c \in \mathcal{C}_{\sigma_{m}}$ and $c \in \mathcal{C}_{\tau_{m}}$.

On the proof of Lemma 18. It is easy to see that our recursive Definition 6 of the length operators $\mathrm{L}_{\sigma, c}$ reduces the task of proving Lemma 18 to the curves that represent a boundary component of $\Sigma_{m}$. Let us write $\mathrm{L}_{\sigma, e} \equiv \mathrm{L}_{\sigma, c(\sigma, e)}$. 
In the cases $m=F_{p q}, S_{p}, T_{p}, Z_{p}$ we will need to verify Lemma 18 by direct calculations. Let us begin with the case $m=F_{p q}$. The claim is again trivial for the length operators associated to the incoming boundary components of $\Sigma_{m}$. Let us therefore focus on the length operators $\mathrm{L}_{\sigma_{m}, f}, \mathrm{~L}_{\tau_{m}, f}$ assigned to the outgoing boundary component of $\Sigma_{m}$. The Definition 6 yields the following expressions for the length operators $\mathrm{L}_{\sigma_{m}, f}$ and $\mathrm{L}_{\tau_{m}, f}$ respectively.

$$
\begin{aligned}
\mathrm{L}_{\sigma_{m}, f}= & e^{\mathrm{z}_{21}+\mathrm{y}_{3}+\mathrm{y}_{2}+\mathrm{y}_{1}}+e^{\mathrm{z}_{21}-\mathrm{y}_{3}+\mathrm{y}_{2}+\mathrm{y}_{1}}+e^{-\mathrm{z}_{21}-\mathrm{y}_{3}-\mathrm{y}_{2}-\mathrm{y}_{1}} \\
& +e^{\mathrm{y}_{1}-\mathrm{y}_{2}-\mathrm{y}_{3}}+e^{\mathrm{y}_{1}+\mathrm{y}_{2}-\mathrm{y}_{3}}+e^{-\mathrm{y}_{1}-\mathrm{y}_{2}-\mathrm{y}_{3}} \\
& +e^{\mathrm{z}_{21}+\mathrm{y}_{2}+\mathrm{y}_{1}} \mathrm{~L}_{3}+e^{\mathrm{y}_{1}-\mathrm{y}_{3}} \mathrm{~L}_{2}+e^{-\mathrm{y}_{2}-\mathrm{y}_{3}} \mathrm{~L}_{1}, \\
\mathrm{~L}_{\tau_{m}, f}= & e^{+\mathrm{z}_{32}^{\prime}+\mathrm{y}_{3}^{\prime}+\mathrm{y}_{2}^{\prime}+\mathrm{y}_{1}^{\prime}}+e^{-\mathrm{z}_{32}^{\prime}-\mathrm{y}_{3}^{\prime}-\mathrm{y}_{2}^{\prime}-\mathrm{y}_{1}^{\prime}}+e^{-\mathrm{z}_{32}^{\prime}-\mathrm{y}_{3}^{\prime}-\mathrm{y}_{2}^{\prime}+\mathrm{y}_{1}^{\prime}} \\
& +e^{\mathrm{y}_{1}^{\prime}+\mathrm{y}_{2}^{\prime}+\mathrm{y}_{3}^{\prime}}+e^{\mathrm{y}_{1}^{\prime}-\mathrm{y}_{2}^{\prime}-\mathrm{y}_{3}^{\prime}}+e^{\mathrm{y}_{1}^{\prime}+\mathrm{y}_{2}^{\prime}-\mathrm{y}_{3}^{\prime}} \\
& +e^{\mathrm{y}_{2}^{\prime}+\mathrm{y}_{1}^{\prime}} \mathrm{L}_{3}^{\prime}+e^{\mathrm{y}_{1}^{\prime}-\mathrm{y}_{3}^{\prime}} \mathrm{L}_{2}^{\prime}+e^{-\mathrm{z}_{32}^{\prime}-\mathrm{y}_{2}^{\prime}-\mathrm{y}_{3}^{\prime}} \mathrm{L}_{1}^{\prime} .
\end{aligned}
$$

We have used the abbreviations $\mathrm{y}_{\iota} \equiv \mathrm{y}_{\varphi_{\sigma}, e_{\iota}}, \mathrm{y}_{\iota}^{\prime} \equiv \mathrm{y}_{\varphi_{\tau}, e_{\iota}}, \mathrm{L}_{\iota} \equiv \mathrm{L}_{\sigma, e_{\iota}}$ and $\mathrm{L}_{\iota}^{\prime} \equiv \mathrm{L}_{\tau, e_{\iota}}$ for $\iota \in$ $\{1,2,3\}$, as well as $\mathrm{z}_{\iota} \equiv \mathrm{z}_{\varphi_{\sigma}, e_{\iota}}, \mathrm{z}_{\iota}^{\prime} \equiv \mathrm{z}_{\varphi_{\tau}, e_{\iota}}$ for $\iota \in\{32,2 \mathbf{1}\}$. The labelling of the edges is the one introduced in Figure 26. According to Proposition 16 we need to calculate

$$
\begin{aligned}
E_{b}\left(\mathrm{z}_{32}^{\prime}\right) \cdot & \left(e^{\mathrm{y}_{3}^{\prime}+\mathrm{y}_{2}^{\prime}+\mathrm{y}_{1}^{\prime}+\mathrm{z}_{32}}+e^{\mathrm{y}_{1}^{\prime}-\mathrm{y}_{2}^{\prime}-\mathrm{y}_{3}^{\prime}}+\right. \\
& +e^{\mathrm{y}_{2}^{\prime}+\mathrm{y}_{1}^{\prime}} \mathrm{L}_{3}^{\prime}+e^{\mathrm{y}_{1}^{\prime}-\mathrm{y}_{3}^{\prime}} \mathrm{L}_{2}^{\prime}+e^{-\mathrm{z}_{32}^{\prime}-\mathrm{y}_{2}^{\prime}-\mathrm{y}_{3}^{\prime} \mathrm{L}_{3}^{\prime}+} \\
& +e^{\frac{1}{2}\left(\mathrm{y}_{1}^{\prime}+\mathrm{y}_{2}^{\prime}-\mathrm{y}_{3}^{\prime}\right)}\left(1+e^{-\mathrm{z}_{32}^{\prime}}\right) e^{\frac{1}{2}\left(\mathrm{y}_{1}^{\prime}+\mathrm{y}_{2}^{\prime}-\mathrm{y}_{3}^{\prime}\right)}+ \\
& \left.+e^{-\frac{1}{2}\left(\mathrm{y}_{1}^{\prime}+\mathrm{y}_{2}^{\prime}+\mathrm{y}_{3}^{\prime}+\mathrm{z}_{32}^{\prime}\right)}\left(1+e^{-\mathrm{z}_{32}^{\prime}}\right) e^{-\frac{1}{2}\left(\mathrm{y}_{1}^{\prime}+\mathrm{y}_{2}^{\prime}+\mathrm{y}_{3}^{\prime}+\mathrm{z}_{32}^{\prime}\right)}\right) \cdot E_{b}\left(\mathrm{z}_{32}^{\prime}\right)^{\dagger}
\end{aligned}
$$

We finally need to apply equation (E.11). The terms in the first line of (E.14) have $N=1$, those in the second line $N=0$, and all other terms have $N=-1$. Straightforward application of equation (E.11) shows that the expression given in (E.14) equals $\mathrm{L}_{\tau_{m}, f}$, as claimed.

The next case we will consider is $m=S_{p}$. It clearly suffices to restrict attention to the case that $\Sigma_{m}=\Sigma_{1,1}$, the one-holed torus. On $\Sigma_{m}$ let us consider the fat graphs $\varphi_{i}, i=1,2,3$ depicted in Figure 27.

We have $\varphi_{1}=\tilde{\varphi}_{m}^{\prime}$ and $\varphi_{3}=\theta_{m}^{\prime}$ respectively. The sequence of fat graph $\left(\varphi_{3}, \varphi_{2}, \varphi_{1}\right)$ defines the path $\pi_{m} \in\left[\theta_{m}^{\prime}, \tilde{\varphi}_{m}^{\prime}\right]$ which we will use. The only relevant curve is the curve $\beta$ which represents the boundary of $\Sigma_{m}$. Definition 6 yields the following expressions for the length
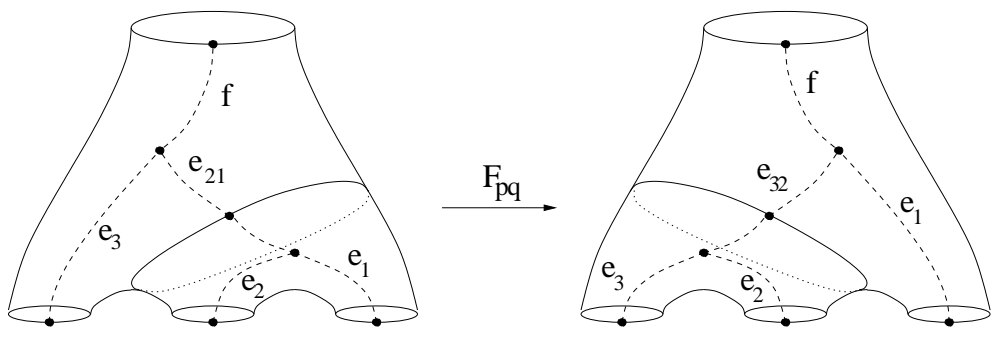

Figure 26: Labelling of the edges of $\sigma_{m}$ for $m=F_{p q}$. 


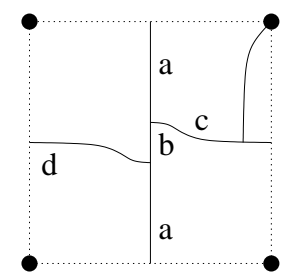

(1)

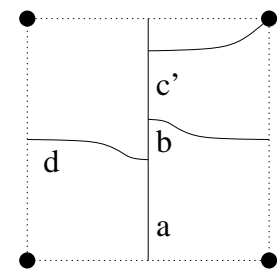

(2)

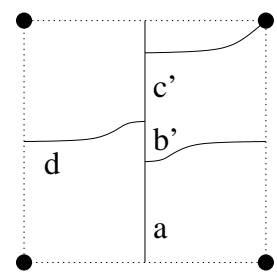

(3)

Figure 27: Fat graphs $\varphi_{i}$ on the one-holed torus.

operators $\mathrm{L}_{\varphi_{1}, \beta}$ and $\mathrm{L}_{\varphi_{3}, \beta}$ respectively:

$$
\begin{aligned}
& \mathrm{L}_{\varphi_{1}, \beta}=e^{\mathbf{z}_{d}-\mathbf{z}_{c}}+2 \cosh \left(\mathbf{z}_{c}+\mathbf{z}_{d}+\mathbf{z}_{a}+\mathrm{z}_{b}\right) \\
& +e^{\mathbf{z}_{d}}\left(1+e^{\mathbf{z}_{b}}+e^{\mathbf{z}_{b}+\mathbf{z}_{a}}\right)+e^{-\mathbf{z}_{c}}\left(1+e^{-\mathbf{z}_{a}}+e^{-\mathbf{z}_{a}-\mathbf{z}_{b}}\right), \\
& \mathrm{L}_{\varphi_{3}, \beta}=e^{\mathbf{z}_{c^{\prime}}-\mathbf{z}_{a}}+2 \cosh \left(\mathbf{z}_{a}+\mathbf{z}_{d}+\mathbf{z}_{c^{\prime}}+\mathrm{z}_{b^{\prime}}\right) \\
& +e^{\mathbf{z}_{c^{\prime}}}\left(1+e^{\mathbf{z}_{b^{\prime}}}+e^{\mathbf{z}_{b^{\prime}}+\mathbf{z}_{d}}\right)+e^{-\mathbf{z}_{a}}\left(1+e^{-\mathbf{z}_{d}}+e^{-\mathbf{z}_{d}-\mathbf{z}_{b^{\prime}}}\right) .
\end{aligned}
$$

The labelling of the relevant edges is the one introduced in Figure 27. With the help of Proposition 16 one may calculate

$$
\begin{aligned}
\mathrm{L}_{\varphi_{2}, \beta} \equiv \mathrm{a}_{\left[\varphi_{2}, \varphi_{1}\right]}\left(\mathrm{L}_{\varphi_{1} ; \beta}\right) & =e^{\mathbf{z}_{c^{\prime}}-\mathbf{z}_{a}}+2 \cosh \left(\mathbf{z}_{a}+\mathbf{z}_{d}+\mathbf{z}_{c^{\prime}}+\mathbf{z}_{b}\right) \\
& +e^{\mathbf{z}_{c^{\prime}}}\left(1+e^{\mathbf{z}_{d}}+e^{\mathbf{z}_{d}+\mathbf{z}_{b}}\right)+e^{-\mathbf{z}_{a}}\left(1+e^{-\mathbf{z}_{b}}+e^{-\mathbf{z}_{b}-\mathbf{z}_{b}}\right) .
\end{aligned}
$$

One may then apply Proposition 16 once more to calculate $a_{\left[\varphi_{3}, \varphi_{2}\right]}\left(L_{\varphi_{2}, \beta}\right)$. The result is $L_{\varphi_{3}, \beta}$ as claimed.

We have furthermore verified by direct calculations that Lemma 18 holds in the cases $m=T_{p}, Z_{p}, B_{p}, B_{p}^{\prime}$ respectively. These calculations proceed along very similar lines as in the previous two cases, which motivates us to omit the details.

In the remaining cases $m=A_{p q}, m=B_{p q}$ and $m=S_{p q}$ one may use the factorization of the chains $\pi_{m}$ which follows from the definitions (13.5), (13.7) and (13.8) respectively in order to reduce the proof to the cases where the result is already established. This completes our discussion of the proof of Lemma 18.

\section{References}

[ADPW] S. Axelrod, S. Della Pietra, E. Witten, Geometric quantization of Chern-Simons gauge theory. J. Differential Geom. 33 (1991) 787-902.

[AGS] A.Yu. Alekseev; H. Grosse, Harald; V. Schomerus, Combinatorial quantization of the Hamiltonian Chern-Simons theory. I. Comm. Math. Phys. 172 (1995) 317-358, II. Comm. Math. Phys. 174 (1996) 561-604,

A.Yu. Alekseev, V. Schomerus, Representation theory of Chern-Simons observables. Duke Math. J. 85 (1996) 447-510

[AMR] J.E. Andersen; J. Mattes; N. Reshetikhin, Quantization of the algebra of chord diagrams. Math. Proc. Cambridge Philos. Soc. 124 (1998) 451-467 
[Ba] E.W. Barnes: Theory of the double gamma function, Phil. Trans. Roy. Soc. A196 (1901) 265-388

[BK1] B. Bakalov, A. Kirillov jun., On the Lego-Teichmüller Game, Transform. Groups 5 (2000) 207-244 [arXiv: math.GT/9809057]

[BK2] B. Bakalov, A. Kirillov jun., Lectures on tensor categories and modular functors. University Lecture Series, 21. American Mathematical Society, Providence, RI, 2001

[BR] E. Buffenoir, Ph. Roche, Link invariants and combinatorial quantization of Hamiltonian Chern-Simons theory. Comm. Math. Phys. 181 (1996) 331-365.

[BT] A. Bytsko, J. Teschner, R-operator, co-product and Haar-measure for the Modular Double of $U_{q}(\mathfrak{s l}(2, \mathbb{R}))$, Comm. Math. Phys. 240 (2003) 171-196, [arXiv: math.QA/0208191]

[CF1] L.O. Checkov, V. Fock: A quantum Teichmüller space, Theor. Math. Phys. 120 (1999) 1245-1259

[CF2] L.O. Checkov, V. Fock: Quantum modular transformations, the pentagon relation, and geodesics, Proc. Steklov Inst. Math. 226 (1999) 149-163

[CP] L.O. Checkov, R. Penner: On Quantizing Teichmüller and Thurston theories, Preprint [arXiv:math.AG/0403247]

[D] M. Dehn: Die Gruppe der Abbildungsklassen, Acta Math. 69 (1938) 135-206

[Fa] L. Faddeev: Modular double of a quantum group. Math. Phys. Stud. 21 (2000) 149-156 [arXiv:math.QA/9912078]

[FG] V.V. Fock, A.B. Goncharov: Cluster ensembles, quantization and the dilogarithm, Preprint [arXiv:math.AG/0311245]

[FuG] L. Funar, R. Gelca: On the groupoid of transformations of rigid structures on surfaces, J.Math.Sci.Univ.Tokyo 6 (1999) 599-646.

[FK] L.D. Faddeev and R.M. Kashaev: Quantum dilogarithm, Mod. Phys. Lett. A9 (1994) 427-434 [arXiv: hep-th/9310070]

[FKV] L. Faddeev, R. Kashaev, A. Volkov: Strongly coupled discrete Liouville theory: Algebraic approach and duality, Comm. Math. Phys. 219 (2001) 199-219 [arXiv:hepth/0006156]

[Fo] V.V. Fock: Dual Teichmüller spaces, [arXiv: hep-th/9702018]

[FR] V.V. Fock, A.A. Rosly, Poisson structure on moduli of flat connections on Riemann surfaces and the r-matrix, in: Morozov, A. Yu. (ed.) et al., Moscow Seminar in mathematical physics. Providence, RI: American Mathematical Society. Transl., Ser. 2, Am. Math. Soc. 191 (43), 67-86 
[Ge] S. Gervais: Presentation and central extension of mapping class groups, Trans. AMS348 (1996) 3097-3132

[Go1] W.M. Goldman, The symplectic nature of fundamental groups of surfaces. Adv. in Math. 54 (1984) 200-225.

[Go2] W.M. Goldman, Topological components of spaces of representations. Invent. Math. 93 (1988), no. 3, 557-607

[GS] V. Guillemin, S. Sternberg, Symplectic techniques in physics, Cambridge University Press, Cambridge, 1990

[Ha] J. Harer: The virtual cohomological dimension of the mapping class group of an orientable surface, Inv. Math. 84 (1986) 157-176

[Hi1] N. J. Hitchin, The self-duality equations on a Riemann surface. Proc. London Math. Soc. (3) 55 (1987), no. 1, 59-126

[Hi2] N. J. Hitchin, Flat connections and geometric quantization. Comm. Math. Phys. 131 (1990) 347-380

[IT] Y. Imayoshi, M. Taniguchi: An introduction to Teichmüller spaces, Springer 1992

[Ka1] R.M. Kashaev: Quantization of Teichmüller spaces and the quantum dilogarithm, Lett. Math. Phys. 43 (1998) 105-115, q-alg/9705021,

[Ka2] R.M. Kashaev: Liouville central charge in quantum Teichmuller theory, Proc. of the Steklov Inst. of Math. Vol.226(1999)63-71, hep-th/9811203

[Ka3] R.M. Kashaev: On the spectrum of Dehn twists in quantum Teichmüller theory, Physics and combinatorics, 2000 (Nagoya), 63-81, World Sci. Publishing, River Edge, NJ, 2001.

[Ka4] R.M. Kashaev, The quantum dilogarithm and Dehn twists in quantum Teichmüller theory. Integrable structures of exactly solvable two-dimensional models of quantum field theory (Kiev, 2000), 211-221, NATO Sci. Ser. II Math. Phys. Chem., 35, Kluwer Acad. Publ., Dordrecht, 2001.

[KaV] R.M. Kashaev, The hyperbolic volume of knots from the quantum dilogarithm. Lett. Math. Phys. 39 (1997) 269-275

[KLS] S. Kharchev, D. Lebedev and M. Semenov-Tian-Shansky: Unitary representations of $\mathcal{U}_{q}(\mathfrak{s l}(2, \mathbb{R}))$, the modular double, and the multiparticle q-deformed Toda chains, Comm. Math. Phys. 225 (2002) 573-609 [arXiv: hep-th/0102180]

[Ko] M. Kontsevich, Intersection theory on the moduli space of curves and the matrix Airy function, Comm. Math. Phys. 147 (1992) 1-23

[La] Y. Laszlo, Hitchin's and WZW connections are the same. J. Differential Geom. 49 (1998) $547-576$ 
[Lu] F. Luo: A Presentation of the mapping class groups, Math. Res. Lett. 4 (1997) 735-739

[MS] G. Moore, N. Seiberg, Classical and quantum conformal field theory, Comm. Math. Phys. 123 (1989) 177-254

[P1] R.C. Penner: The Decorated Teichmüller Space of Punctured Surfaces, Comm. Math. Phys. 113 (1987) 299-339

[P2] R.C. Penner: Weil-Petersson Volumes J. Differential Geometry 35 (1992) 559-608

[P3] R.C. Penner: Universal Constructions in Teichmüller Theory, Adv. Math. 98 (1993) 143215

[PT] B.Ponsot and J.Teschner. Clebsch-Gordan and Racah-Wigner coefficients for a continuous series of representations of $\mathcal{U}_{q}(\mathfrak{s l}(2, \mathbb{R}))$, Comm. Math. Phys. 224 (2001) 613-655

[RT] N. Reshetikhin, V.G. Turaev, Invariants of 3-manifolds via link polynomials and quantum groups. Invent. Math. 103 (1991) 547-597

[Ru] S.N.M. Ruijsenaars: First order analytic difference equations and integrable quantum systems, J. Math. Phys. 38 (1997) 1069-1146.

[Se] I.E. Segal: Transforms for operators and symplectic automorphisms over a locally compact abelian group, Math. Scand. 13 (1963) 31-43.

[Sh] T. Shintani: On a Kronecker limit formula for real quadratic fields, J. Fac. Sci. Univ. Tokyo Sect. 1A Math. 24 (1977) 167-199

[So] C. Sorger, La formule de Verlinde. Séminaire Bourbaki, Vol. 1994/95. Astérisque No. 237 (1996), Exp. No. 794, 3, 87-114

[TT] L.A. Takhtajan, 1.-P. Teo: Quantum Liouville theory in the background field formalism I. Compact Riemann surfaces Commun.Math.Phys. 268 (2006) 135-197

[TL1] J. Teschner, Liouville theory revisited. Classical Quantum Gravity 18 (2001), no. 23, R153-R222

[TL2] J. Teschner, A lecture on the Liouville vertex operators., Int. J. Mod. Phys. A, Supplement, Vol. 19 (2004) 436-458

[TT2] J. Teschner, An analog of a modular functor from quantized Teichmüller theory, II, in preparation

[Tu] V. Turaev, Quantum Invariants of Knots and 3-manifolds, W. de Gruyter, Berlin 1994.

[V] H. Verlinde, Conformal field theory, two-dimensional quantum gravity and quantization of Teichmüller space. Nuclear Phys. B 337 (1990) 652-680.

[Vo] A.Yu. Volkov: Noncommutative hypergeometry, Comm. Math. Phys. 258 (2005) 257-273 
[Wa] N. Wallach, Real Reductive Groups II, Academic Press, Inc., Boston, MA, 1992.

[Wi] E. Witten, Quantum field theory and the Jones polynomial. Comm. Math. Phys. 121 (1989) 351-399

[Wo1] S.A. Wolpert, On the symplectic geometry of deformations of a hyperbolic surface Ann. of Math. 117 (1983) 207-234

[Wo2] S.A. Wolpert, On the Weil-Petersson geometry of the moduli space of curves Amer. J. Math 107 (1985) 969-997

[Wo3] S.A. Wolpert, Geodesic length functions and the Nielsen problem J. Differential Geom. 25 (1987) 275-296

[Wor] S.L. Woronowicz: Quantum exponential function, Rev. Math. Phys. 12 (2000) 873-920 